\title{
Low Overhead Memory Subsystem Design for a Multicore Parallel DSP Processor
}

Jian Wang

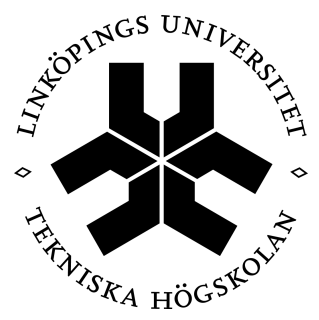

Department of Electrical Engineering

Linköping University

SE-581 83 Linköping, Sweden

Linköping 2014

ISBN 978-91-7519-556-8

ISSN 0345-7524 
Low Overhead Memory Subsystem Design

for a Multicore Parallel DSP Processor

Jian Wang

ISBN 978-91-7519-556-8

Copyright (c) Jian Wang, 2014

Linköping Studies in Science and Technology

Dissertation No. 1532

ISSN 0345-7524

Department of Electrical Engineering

Linköping University

SE-581 83 Linköping

Sweden

Phone: +4613281000

Author e-mail: jiawa001@gmail.com

\section{Cover image}

Combined Star and Ring onchip interconnection of the ePUMA multicore DSP.

Parts of this thesis are reprinted with permission from the IEEE.

Printed by UniTryck, Linköping University

Linköping, Sweden, 2014 


\section{Abstract}

The physical scaling following Moore's law is saturated while the requirement on computing keeps growing. The gain from improving silicon technology is only the shrinking of the silicon area, and the speedpower scaling has almost stopped in the last two years. It calls for new parallel computing architectures and new parallel programming methods.

Traditional ASIC (Application Specific Integrated Circuits) hardware has been used for acceleration of Digital Signal Processing (DSP) subsystems on SoC (System-on-Chip). Embedded systems become more complicated, and more functions, more applications, and more features must be integrated in one ASIC chip to follow up the market requirements. At the same time, the product lifetime of a SoC with ASIC has been much reduced because of the dynamic market. The life time of the design for a typical main chip in a mobile phone based on ASIC acceleration is about half a year and the NRE (Non-Recurring Engineering) cost of it can be much more than 50 million US\$.

The current situation calls for a new solution than that of ASIC. ASIP (Application Specific Instruction set Processor) offers comparable power consumption and silicon cost to ASICs. Its greatest advantage is the functional flexibility in a predefined application domain. ASIP based SoC enables software upgrading without changing hardware. Thus the product life time can be 5-10 times more than that of ASIC based SoC.

This dissertation will present an ASIP based SoC, a new unified parallel DSP subsystem named ePUMA (embedded Parallel DSP Platform 
with Unique Memory Access), to target embedded signal processing in communication and multimedia applications. The unified DSP subsystem can further reduce the hardware cost, especially the memory cost, of embedded SoC processors, and most importantly, provide full programmability for a wide range of DSP applications. The ePUMA processor is based on a master-slave heterogeneous multi-core architecture. One master core performs the central control, and multiple Single Instruction Multiple Data (SIMD) coprocessors work in parallel to offer a majority of the computing power.

The focus and the main contribution of this thesis are on the memory subsystem design of ePUMA. The multi-core system uses a distributed memory architecture based on scratchpad memories and software controlled data movement. It is suitable for the data access properties of streaming applications and the kernel based multi-core computing model. The essential techniques include the conflict free access parallel memory architecture, the multi-layer interconnection network, the non-address stream data transfer, the transitioned memory buffers, and the lookup table based parallel memory addressing. The goal of the design is to minimize the hardware cost, simplify the software protocol for inter-processor communication, and increase the arithmetic computing efficiency.

We have so far proved by applications that most DSP algorithms, such as filters, vector/matrix operations, transforms, and arithmetic functions, can achieve computing efficiency over $70 \%$ on the ePUMA platform. And the non-address stream network provides equivalent communication bandwidth by less than $30 \%$ implementation cost of a crossbar interconnection. 


\section{Preface}

This thesis includes the following papers published during my research from August 2008 to July 2013:

- Jian Wang, Andréas Karlsson, Joar Sohl, and Dake Liu. Convolutional decoding on deep-pipelined SIMD processor with flexible parallel memory. In Digital System Design (DSD), 2012, pages 529532. IEEE, 2012.

- Jian Wang, Joar Sohl, Andréas Karlsson, and Dake Liu. An efficien$\mathrm{t}$ streaming star network for multi-core parallel DSP processor. In Proceedings of the 2011 Second International Conference on Networking and Computing, ICNC '11, pages 332-336, Washington, DC, USA, 2011. IEEE Computer Society.

- Jian Wang, Andréas Karlsson, Joar Sohl, Magnus Pettersson, and D. Liu. A multi-level arbitration and topology free streaming network for chip multiprocessor. In ASIC (ASICON), 2011 IEEE 9th International Conference on, pages 153-158, oct. 2011.

- Jian Wang, Joar Sohl, and Dake Liu. Architectural support for reducing parallel processing overhead in an embedded multiprocessor. In Proceedings of the 2010 IEEE/IFIP International Conference on Embedded and Ubiquitous Computing, EUC '10, pages 47-52, Washington, DC, USA, 2010. IEEE Computer Society.

- Jian Wang, Joar Sohl, Olof Kraigher, and Liu Dake. Software programmable data allocation in multi-bank memory of simd proces- 
sors. In Proceedings of the 2010 13th Euromicro Conference on Digital System Design: Architectures, Methods and Tools, pages 28-33. IEEE Computer Society, 2010.

- Jian Wang, Joar Sohl, Olof Kraigher, and Dake Liu. ePUMA: A novel embedded parallel DSP platform for predictable computing. In Education Technology and Computer (ICETC), 2010 2nd International Conference on, volume 5, pages V5-32 -V5-35, june 2010.

I am also co-author of the following publications:

- Joar Sohl, Jian Wang, Andréas Karlsson, and Dake Liu. Automatic permutation for arbitrary static access patterns. In Parallel and Distributed Processing with Applications (ISPA), 2012 IEEE 10th International Symposium on, pages 215-222, july 2012.

- Dake Liu, Andréas Karlsson, Joar Sohl, Jian Wang, Magnus Petersson, and Wenbiao Zhou. ePUMA embedded parallel DSP processor with unique memory access. In Information, Communications and Signal Processing (ICICS) 2011 8th International Conference on, pages 1-5, dec. 2011.

- Dake Liu, Joar Sohl, and Jian Wang. Parallel computing and it$\mathrm{s}$ architecture based on data access separated kernels. IJERTCS, International Journals Embedded and Real-Time Communication systems.

- Joar Sohl, Jian Wang, and Dake Liu. Large matrix multiplication on a novel heterogeneous parallel DSP architecture. In Proceedings of the 8th International Symposium on Advanced Parallel Processing Technologies, APPT '09, pages 408-419, Berlin, Heidelberg, 2009. Springer-Verlag. 


\section{Acknowledgments}

I would like to take this opportunity to express my gratitude to many people for their support during my study at Linköping university. I would like to thank the following:

- Professor Dake Liu for the opportunity to work on this interesting project and for the advices during my study.

- Professor Torkel Glad for the support and supervision in the final year of my study.

- The head of Computer Engineering division Tomas Svensson for the support and encouragement during my time at the division.

- My co-supervisor Dr. Andreas Ehliar for the advices in technical details and guide in writing this thesis.

- PhD candidates Joar Sohl and Andréas Karlsson for great cooperation in the ePUMA project, for all the fruitful discussions and collaboration in publications. And all $\mathrm{PhD}$ students I have met during my study for the comments and ideas about my work.

- Ylva Jernling for your help in administration affairs, Olle Seger for the help in teaching and the valuable comments on this dissertation, and Anders Nilsson for the support in computer and software. And everyone else at Computer Engineering division, it has been a great time to work with you all. 
- Dr. Di Wu for all interesting discussions, valuable suggestions in my research and my career, and for the friendship.

- All my other friends for the good time at Linköping.

- My wife Fan Zhang for her love, support, encouragement, and understanding during our time in Sweden.

- My mother Zhongxia Yang, my sister Wenjing Wang and her husband Runlong Gong, and all my families for their constant support and encouragement.

This research was supported by the Swedish Foundation for Strategic Research (SSF).

Jian Wang

Lund, March 2014 


\section{Contents}

Abbreviations $\quad$ Xv

I Background 1

1 Introduction 3

1.1 Motivation ..................... 5

1.1.1 Programmable Multicore DSP . . . . . . . . 5

1.1.2 DRAM and SPM Based Memory Subsystem . . . . 6

1.1.3 Parallel Local Memory Architecture . . . . . . . . . 7

1.1.4 Design Goal and Challenge . . . . . . . . . . 8

1.2 Contributions .................. 10

1.3 Thesis Organization . . . . . . . . . . . . . . 12

2 High Performance Streaming Computing 15

2.1 Computing Characteristics . . . . . . . . . . . . . . . 15

2.2 Computation Kernels . . . . . . . . . . . . . . . . . . . . . 18

2.3 Data Flow Based Signal Processing . . . . . . . . . . . . . 21

2.3.1 LTE Uplink Baseband Signal Processing . . . . . . . 21

2.3.2 H.264 Decoder . . . . . . . . . . . . . 22

2.3.3 3D Rendering Pipeline . . . . . . . . . . . . . . 23

3 Parallel Processor Architectures $\quad 25$

3.1 Introduction . . . . . . . . . . . . . . 25

3.2 Instruction Level Parallel Execution . . . . . . . . . . . 26 
3.2.1 Superscalar . . . . . . . . . . . . 26

3.2 .2 VLIW . . . . . . . . . . . . . . . . . . 26

3.3 SIMD Architecture for Data Level

Parallelism . . . . . . . . . . . . . . . . . 28

3.3.1 SIMD Arithmetic Functions . . . . . . . . . . . . 28

3.3.2 SIMD Data Accesses . . . . . . . . . . . . . . . . . 29

3.3.3 SWAR Extension vs. SIMD Engine . . . . . . . . . . 31

3.4 Chip Multiprocessor . . . . . . . . . . . . . . . . . . 31

3.4.1 Multicore Computing Model . . . . . . . . . . . 32

3.4.2 Shared Memory vs. Distributed Memory . . . . . 34

3.4.3 Interconnection Architecture . . . . . . . . . . . 36

3.5 Performance Obstacles . . . . . . . . . . . . . . . . . . . 38

3.5.1 Streaming Data Movement . . . . . . . . . . . . 39

3.5.2 Parallel Data Access for a SIMD Datapath . . . . . . 40

3.5.3 DRAM Memory Bandwidth Efficiency . . . . . . . . 41

3.5.4 Memory Coherency Overhead . . . . . . . . . . 43

3.5.5 Control Overhead . . . . . . . . . . . . . . . 44

3.6 Programmable Architectures for Streaming Applications . 45

3.6.1 Imagine . . . . . . . . . . . . . . . 45

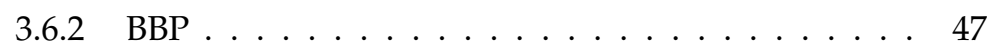

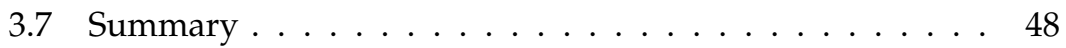

4 Parallel Memory and Conflict Free Data Access 49

4.1 Introduction . . . . . . . . . . . . . . . . . . . . 49

4.2 Multi-bank Memory Architecture . . . . . . . . . . . . . 51

4.2.1 Fundamental Concepts . . . . . . . . . . . . 51

4.2.2 Parallel Memory Architecture . . . . . . . . . . . 53

4.3 Conflict Free Parallel Memory Access . . . . . . . . . . . 55

4.4 Lookup-Table Based Address Generation . . . . . . . . . 56

4.5 Summary . . . . . . . . . . . . . . . . . . 57 
II System Architecture

5 ePUMA: A Multicore DSP Architecture for Streaming Signal Processing $\quad 61$

5.1 System Overview . . . . . . . . . . . . . . . . . . . . 61

5.2 Architecture Design Decisions . . . . . . . . . . . . . . . 62

5.3 Multicore Computing Models on ePUMA . . . . . . . . 68

5.4 Inter-Processor Data Communication . . . . . . . . . . 70

5.4.1 Data Memory Placement . . . . . . . . . . . . . . 70

5.4.2 Stream Data Movement . . . . . . . . . . . . . . 71

5.4.3 Notification and Synchronization . . . . . . . . . . 72

5.5 Host Interface to DSP Subsystem . . . . . . . . . . . . . 73

5.5.1 Standalone Application . . . . . . . . . . . . . 74

5.5 .2 Standard API Interface . . . . . . . . . . . . . . . . 74

5.5.3 Multitask Execution . . . . . . . . . . . . . . 75

5.6 Support for Debug . . . . . . . . . . . . . . 75

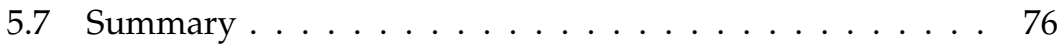

6 Memory Subsystem Design 77

6.1 Introduction . . . . . . . . . . . . . 77

6.2 Memory Hierarchy . . . . . . . . . . . . . . . . 77

6.3 Multi-Layer Interconnection Network . . . . . . . . . . 79

6.3.1 Stream Data Transfer . . . . . . . . . . . . . . . . . 80

6.4 Star Network . . . . . . . . . . . . . . . . . . . . . . . 82

6.4 .1 Data Streams . . . . . . . . . . . . . . . . . 83

6.4.2 Hardware Architecture . . . . . . . . . . . . 85

6.5 Ring Network . . . . . . . . . . . . . . . . . . . . . . 87

6.5 .1 Data Streams . . . . . . . . . . . . . . . . . . . 88

6.5.2 Hardware Architecture . . . . . . . . . . . . 88

6.5.3 Communication Protocol . . . . . . . . . . . . . . . 91

6.6 Serial Bus . . . . . . . . . . . . . . . . . . . 92

6.6.1 Hardware Architecture . . . . . . . . . . . . . 93

6.6.2 Communication Protocol . . . . . . . . . . . . . 94

6.7 DMA Controller . . . . . . . . . . . . . . . . . . . . . . 94 
6.7.1 Task Configuration . . . . . . . . . . . . . . . . . 94

6.7 .2 Hardware Task Queue . . . . . . . . . . . . . . . . 97

6.7 .3 DMA Control Flow . . . . . . . . . . . . . . . . 98

6.8 SIMD Local Memory . . . . . . . . . . . . . . . . . . 101

6.8.1 Processing Units and Scratchpad Memories . . . . . 101

6.8.2 Transitioned LVM Buffers . . . . . . . . . . . . . 103

6.8.3 SIMD Accelerator Extension . . . . . . . . . . . 104

6.9 Stream Communication Scheduling . . . . . . . . . . . . . . . . . . . . . . . . 106

6.10 Summary . . . . . . . . . . . . . . . . . . . . 107

7 Software Development and Toolchain Support 109

7.1 Kernel Based Parallel Programming . . . . . . . . . . . 110

7.1 .1 Master Program . . . . . . . . . . . . . . . . 111

7.1.2 Kernel Scalar Control Code . . . . . . . . . . . . 112

7.1.3 Kernel SIMD code . . . . . . . . . . . . . . . . 115

7.2 ePUMA Simulator . . . . . . . . . . . . . . . . 116

7.2.1 Simulator core . . . . . . . . . . . . . . . . 116

7.2.2 Simulator interface . . . . . . . . . . . . . . . 118

7.3 Software Development Flow . . . . . . . . . . . . . . . . 119

7.3.1 PCAM Parallel Algorithm Design Methodology . . 119

7.3 .2 ePUMA Software Project . . . . . . . . . . . 120

7.4 Multitasking Framework . . . . . . . . . . . . . . 121

7.4 .1 ePUMA Simple OS . . . . . . . . . . . . . . . . . . . . . . . . . . . . . . . . . . . . . . .

7.5 Summary . . . . . . . . . . . . . . . . . . . 125

\section{Results and Conclusions $\quad 127$}

8 Application Implementations 129

8.1 The BLAS syrk Routine . . . . . . . . . . . . . . . . . . 129

$8.264 \times 64$ Matrix Multiplication . . . . . . . . . . . . . . 133

8.2.1 Task Distribution on Multiprocessors . . . . . . . 133

8.2.2 Task Scheduling . . . . . . . . . . . . . . . . . . 134

8.3 Convolutional Decoder . . . . . . . . . . . . . . . . . . 136 
8.3.1 Encoder . . . . . . . . . . . . . . . . . 136

8.3.2 Viterbi Algorithm . . . . . . . . . . . . . . . . 137

8.3.3 Implementation on ePUMA . . . . . . . . . . . . . 140

8.3.4 Performance Comparison . . . . . . . . . . . . . 144

8.4 LTE Downlink Baseband Signal Processing . . . . . . . . . 146

8.4.1 Signal Processing Flow and Computing Kernels . . 146

8.4.2 Addressing Complexity in SIMD Computing . . . . 148

8.4.3 Addressing Complexity in Stream Communication 150

8.4 .4 Performance Evaluation . . . . . . . . . . . . . . . 153

8.5 Other Applications on ePUMA . . . . . . . . . . . . . . 155

9 Performance and Area Evaluation 157

9.1 SIMD Computing Efficiency . . . . . . . . . . . . . . 157

9.2 Memory Subsystem Implementation Cost . . . . . . . . . 158

9.3 System Implementation Cost . . . . . . . . . . . . . . 159

10 Conclusions and Future Work 163

$\begin{array}{ll}\text { Bibliography } & 167\end{array}$

$\begin{array}{ll}\text { IV Appendix } & 177\end{array}$

A Master C Library 179

B SIMD Scalar Controller C Library 187 


\section{Abbreviations}

ACC Accelerator

ACS Add-Compare-Select

AGU Address Generation Unit

ALU Arithmetic and Logic Unit

AMBA Advanced Microcontroller Bus Architecture

API Application Programming Interface

ASIP Application Specific Instruction Set Processor

BBP Baseband Processor

BLAS Basic Linear Algebra Subprograms

CABAC Context-Adaptive Binary Arithmetic Coding

CAVLC Context-Adaptive Variable-Length Coding

CMOS Complementary Metal"COxide "CSemiconductor

CMP Chip Multiprocessor

CP Cyclic Prefix

CPU Central Processing Unit

CUBLAS CUDA Basic Linear Algebra Subroutines

CUDA Compute Unified Device Architecture

DCT Discrete Cosine Transform

DDR Double Data Rate (SDRAM)

DFE Digital Front-End

DLP Data Level Parallelism

DMA Direct Memory Access

DRAM Dynamic Random-Access Memory 


$\begin{array}{ll}\text { DSP } & \text { Digital Signal Processing or Digital Signal Processor } \\ \text { DVB } & \text { Digital Video Broadcasting } \\ \text { FCRAM } & \text { Fast-Cycle Random Access Memory } \\ \text { FEC } & \text { Forward Error Correction } \\ \text { FFT } & \text { Fast Fourier Transform } \\ \text { FIFO } & \text { First In First Out } \\ \text { FIR } & \text { Finite Impulse Response } \\ \text { GOPS } & \text { Giga Operations Per Second } \\ \text { GPS } & \text { Global Positioning System } \\ \text { GPU } & \text { Graphics Processing Unit } \\ \text { GSM } & \text { Global System for Mobile Communications } \\ \text { GUI } & \text { Graphics User Interface } \\ \text { HD } & \text { High Definition } \\ \text { IDCT } & \text { Inverse Discrete Cosine Transform } \\ \text { IDFT } & \text { Inverse Discrete Fourier Transform } \\ \text { IFFT } & \text { Inverse Fast Fourier Transform } \\ \text { IIR } & \text { Infinite Impulse Response } \\ \text { ILP } & \text { Instruction Level Parallelism } \\ \text { IP } & \text { Intellectual Property } \\ \text { ISA } & \text { Instruction Set Architecture } \\ \text { ITRS } & \text { International Technology Roadmap for Semiconductors } \\ \text { LDPC } & \text { Low-Density Parity-Check code } \\ \text { LPDDR } & \text { Low power DDR } \\ \text { LSB } & \text { Least Significant Bits } \\ \text { LTE } & \text { Long Term Evolution } \\ \text { LUT } & \text { Lookup Table } \\ \text { LVM } & \text { Local Vector Memory } \\ \text { MAC } & \text { Multiply and Accumulation } \\ \text { MIMO } & \text { Multiple-Input and Multiple-Output } \\ \text { MIPS } & \text { Million Instructions Per Second } \\ \text { MM } & \text { Main Memory } \\ \text { NI } & \text { Network Interface } \\ \text { NOC } & \text { Network on Chip } \\ & \end{array}$




$\begin{array}{ll}\text { NCC } & \text { Network Channel Control } \\ \text { OFDM } & \text { Orthogonal Frequency-Division Multiplexing } \\ \text { OS } & \text { Operating System } \\ \text { PCAM } & \text { Partition, Communication, Agglomeration, Mapping } \\ \text { PCB } & \text { Process Control Block } \\ \text { PDCCH } & \text { Physical Downlink Control Channel } \\ \text { PHY } & \text { Physical } \\ \text { PPU } & \text { Packet Processing Unit } \\ \text { PM } & \text { Program Memory } \\ \text { PT } & \text { Permutation Table } \\ \text { RAM } & \text { Random-Access Memory } \\ \text { RISC } & \text { Reduced Instruction Set Computing } \\ \text { RLDRAM } & \text { Reduced Latency DRAM } \\ \text { RTL } & \text { Register-Transfer Level } \\ \text { SC } & \text { Scalar Controller } \\ \text { SC-FDMA } & \text { Single Carrier Frequency Division Multiple Access } \\ \text { SDR } & \text { Software Defined Radio } \\ \text { SDRAM } & \text { Synchronous Dynamic Random Access Memory } \\ \text { SIMD } & \text { Single Instruction Multiple Data } \\ \text { SIMT } & \text { Single Instruction Stream Multiple Thread } \\ \text { SPM } & \text { Scratch Pad Memory } \\ \text { SRAM } & \text { Static Random-Access Memory } \\ \text { SVD } & \text { Singular Value Decomposition } \\ \text { SWAR } & \text { SIMD With A Register } \\ \text { TCM } & \text { Tightly Coupled Memroy } \\ \text { TLP } & \text { Task Level Parallelsim } \\ \text { UE } & \text { User End } \\ \text { UMTS } & \text { Universal Mobile Telecommunications System } \\ \text { VAGU } & \text { Vector Address Generation Unit } \\ \text { VLIW } & \text { Very Long Instruction Word } \\ \text { VMAC } & \text { Vector Multiply and Accumulate } \\ \text { VPE } & \text { Vector Processing Engine } \\ & \end{array}$




\section{Part I}

\section{Background}





\section{Chapter 1 Introduction}

Today, most people have witnessed the rapid growth in digital signal processing technology and are enjoying the convenience that the new technologies have brought to our daily life. We have witnessed the mobile handset being developed from the GSM phones in the 1990's for low bitrate audio communication to the latest smartphones in 2013 which support realtime video calls plus many other integrated functions such as fast web browsing using broadband wireless network, HD video recording and playback, GPS navigation, and 3D gaming. The new signal processing technologies have also enabled intelligent driving assistance in Automotive industry, where radar and camera signals are processed to extract useful information by the embedded computing system which could generate proper controls to the car within a shorter time than human reaction.

Behind these advances of new technologies is the ever increasing computing capability of embedded systems. Figure 1.1 lists the computing requirements of typical embedded applications for multimedia, graphics and communication signal processing [1]. It can be seen that a modern mobile device with the aforementioned features would require more than 100 GOPS computing power. This brings a great challenge to the design of embedded DSP systems to meet the performance requirement. Moreover, other requirements such as power consumption and hardware implementation cost became more and more important 


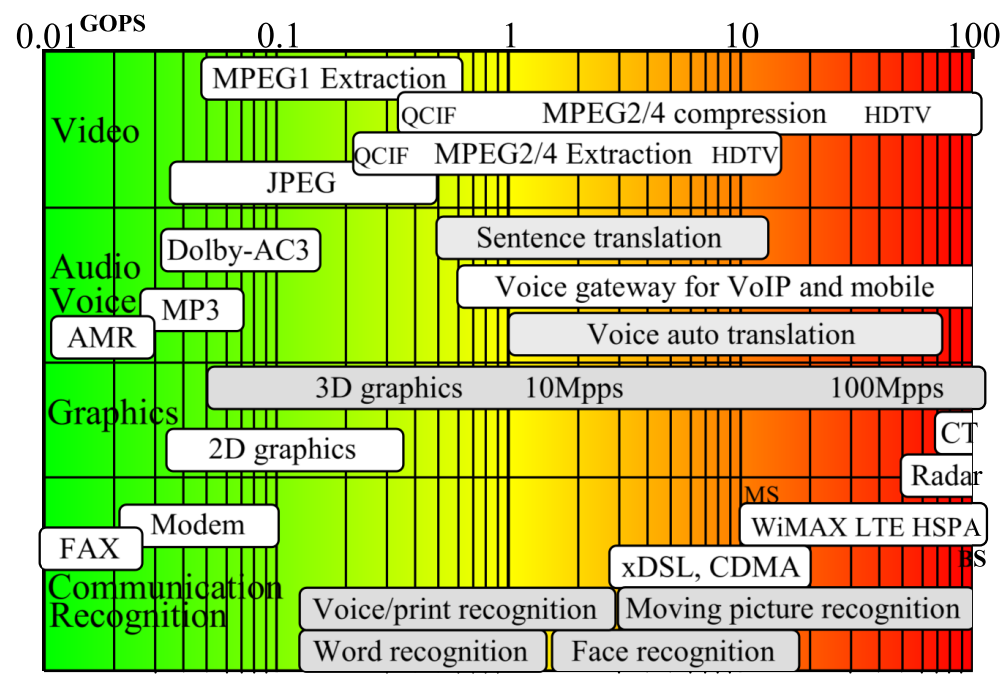

Figure 1.1: Computing throughput of embedded applications [1] (C)2011 IEEE

while the capability and complexity of embedded system grows. The traditional solution of keeping adding hardware components to increase computing capability is no longer power efficient and cost effective. Embedded systems tend to use more programmable components and high degree hardware multiplexing for digital signal processing. Embedded processors include general purpose RISC cores (e.g. ARM [2] and MIPS [3]), DSP processors (e.g. TI64xx [4]) for signal processing, and ASIP processors (e.g. BBP [5]) with a tailored instruction set to benefit a specific application. Hardware multiplexing, also known as reconfigurable computing, is to use multiplexers to switch the functionality and re-use hardware resources. One example is the multi-mode interleaver for FEC in baseband signal processing [6]. Both the programmable and reconfigurable approaches have the same goal of offering computing capacity and flexibility with minimum silicon cost. 


\subsection{Motivation}

\subsubsection{Programmable Multicore DSP}

This thesis work focus on a programmable solution using a multicore DSP subsystem for embedded signal processing. Programmable solutions with a multicore DSP subsystem is the trend in embedded signal processing platforms. Integration of multiple DSP cores and using parallel programming models on such platforms are available in DSP and graphics products. For example, the OpenCL parallel programming model [7] is supported on Cell BE [8] and TI's C6000 multicore DSPs [9]. Almost all desktop GPUs and some mobile graphics engines support CUDA [10] or OpenCL on their unified multi-shader core architecture. A list of processor products that are conformant to OpenCL can be found in [11].

In general, a programmable architecture has the following advantages:

- High Flexibility The programmable solution offers flexibility for multi-mode execution, software upgrade, and bug fix. It reuses the same hardware, the functionality is changed in software.

- Short Time-to-Market It requires less time than custom hardware solutions. Hardware debugging is the most time consuming part in system development. Also, by using a processor simulation model, the software development could start in parallel with hardware integration.

- High Chip Volume and Long Product Lifetime Chip volume is higher than custom hardware because the same product is used for multi-modes. The product lifetime is longer because of the flexibility for software upgrade.

Moreover, using multicore DSP subsystem in a SoC platform has the following benefits: 
- High Hardware Utilization The multicore DSP subsystem uses programmable cores to handle a wide range of DSP computing tasks by the same hardware and supports concurrent computing by multitasking to achieve high hardware utilization.

- Low Implementation Cost Compared to the modern ASIC based SoC platform which typically contains tens of application specific hardware blocks and dedicated data buffers, generalized DSP subsystems can achieve better hardware efficiency and lower memory usage.

- Simplify System Integration A DSP subsystem usually has a common interface to the system interconnection. It reduces the bus complexity by a smaller number of master/slave ports compared to the ASIC based SoC. It not only reduces the hardware implementation cost of the system bus, but also increases the running frequency and therefore the on-chip bandwidth.

- Accelerate Applications Programmable DSP subsystems can accelerate CPU applications by using a parallel programming interface, which is not available in fixed function ASICs.

\subsubsection{DRAM and SPM Based Memory Subsystem}

Due to the large volume of data involved in computing, most DSP systems employ a DRAM based memory subsystem, which consists of offchip DRAM, memory controller, on-chip interconnection, and on-chip local memory. There is a gap between the processor core and the data in the external memory. A common approach in processor architecture design is to use a cache to bridge this gap. But typical DSP algorithms do not have good temporal locality and have non-linear spatial locality which means the DSP architectures cannot benefit much from using a conventional cache. In a DSP application, the input data of an algorithm are usually only used once and then transformed or computed to another data set to be used as input of the following computing step. A typical 
DSP algorithm contains stride based data access patterns, which requires transferring and storing redundant data in the cacheline. Therefore the on-chip memory utilization efficiency is low. The lowest memory utilization efficiency happens when the stride is larger than the cacheline size, which is typically 64 bytes, then the whole 64 bytes on-chip space is used for a single data element. Using scratch pad memory (SPM) in DSP subsystem can achieve better memory utilization by software controlled data movements. This approach relies on DMA engines to move data blocks between off-chip DRAM and on-chip SPM during computing. Application software can use double or triple buffering to hide communication latency.

\subsubsection{Parallel Local Memory Architecture}

The on-chip memory bandwidth is another important factor that limits the computing performance. Almost all DSP cores utilize a parallel datapath, such as VLIW [12], SIMD [13], or a combination of these two architectures [14], in order to achieve high computing throughput. The parallel architectures require a high memory bandwidth to provide computing data in parallel to the datapath. In a shared memory system, multiple DSP cores share the same on-chip memory bandwidth. A memory arbiter is required to grant the memory to different DSP cores to perform read or write operations at different times. This reduces the memory bandwidth for each processing core. Multicore DSP system can implement multiple on-chip memory banks to reduce memory conflict. However, memory coherency in shared memory architecture requires extra software management or hardware snooping logic. It also involves overhead in time while performing synchronization. Another approach is to use distributed memory architecture, which has dedicated on-chip memory for each processor core. The memory bandwidth is only shared between the local processor core and the data communication interface. DSP systems using cache also implement dedicated L1-caches for each DSP core to provide higher bandwidth from the local memory. 
The complex parallel data access patterns from application algorithms can further increase the overhead in shuffling data for parallel computing. This is due to the limitation on the interface of local memory, which usually provides wide data access to the same wordline of a SRAM. Conventional data parallel architecture performs the data shuffle or permutation operations in vector registers after loading from local memory. The execution time of data shuffle instructions can introduce overhead to the true computation time as high as $80 \%$ [15]. Furthermore, the required vector register file size is larger in parallel DSP processors than normal RISC CPU.

A novel approach of using parallel memory modules to provide flexible parallel data access directly to the computing data path is proposed and studied thoroughly in theory by Michael Gossel et al. in [16]. The implementation of the parallel memory architecture is realized in [17]. This parallel memory architecture is suitable for computing algorithms where the data access patterns are predictable.

\subsubsection{Design Goal and Challenge}

The goal of the thesis work is to design a memory architecture and interconnection network of a multicore DSP subsystem to achieve high communication efficiency and minimize on-chip memory usage. The work includes two parts: the recognition of performance obstacles in SoC components and the design of the multi-core DSP local memory subsystem.

The DSP subsystem works together with other hardware modules in a SoC system. It is not beneficial to change the system components, for example the DRAM controller, to serve better only for the DSP subsystem. In a DRAM based system, both the memory controller and the interconnection network have great impact on the system performance. Typical DRAM bandwidth utilization efficiency can vary from $50 \%$ to $80 \%$ depending on the selected DRAM device, the memory controller design, and the data access commands [18]. The development of on-chip interconnection networks has improved the bus performance to approach 
a theoretical bandwidth utilization of $100 \%$ by supporting back-to-back data transfer with no protocol overhead. However, the high performance is gained at the cost of extra hardware on network data buffers and concurrent communication links. Such a network has bad scalability, its size grows rapidly when connecting a large number of cores in the system. The clock speed of the network also decreases when more components are connected to the system bus. One important design trend in both DRAM device and on-chip network to improve bandwidth efficiency is to increase the burst size. A burst is the basic block of continuous memory data transferred by one data access request. Both the memory controller and the system bus have a favor towards accessing data stored continuously in memory. However, multicore parallel computing algorithms tend to divide the data and distribute the computing on multiple processors to execute in parallel. With each DSP core sending random data requests of small bursts at different memory locations, the system bandwidth utilization efficiency will be reduced.

We focus on predictable signal processing algorithms where the data access patterns are known before execution. For predictable computing, we can program the DMA engine to schedule the data communication streams and the data allocation in on-chip parallel memory to hide communication latency as well as increase parallel data access bandwidth. This thesis proposes a unique memory subsystem architecture design and its corresponding programming model.

The target application fields of our multicore DSP include multimedia, graphics, and communication. It focuses on the streaming signal processing applications in these three fields, which is also known as the frontend signal processing in [19]. Backend computing on data contents, for example target recognition and database operations, is not within the scope of this thesis. The thesis investigates various application algorithms and existing processor architecture solutions. The computing analysis has its focus on computing intensive kernels and data access patterns. The processor architecture study focuses on different parallel architectures. 
The thesis work is part of the ePUMA project at Linköping University. The goal of this project is to design a parallel DSP platform for the streaming applications in multimedia, graphics and communication. The focus of this thesis is on the memory subsystem design. The major motivation is to design a low overhead memory subsystem for the proposed multicore parallel DSP platform. The goal of low overhead refers to two aspects, performance and cost. The memory subsystem design should minimize data communication overhead to increase computing performance. It should also have minimal hardware implementation cost.

\subsection{Contributions}

The major contributions of this thesis are listed as below:

Investigate the computing characteristics of streaming DSP. The computing characteristics of streaming signal processing applications are studied in this thesis. It investigates the computation intensive kernels and the data access features including the data type, control flow, and data flow. The streaming applications are mostly based on a data flow of multiple computing stages. The main computing part of each computing stage consists of computing kernels which are dominated by predictable computing. The memory access patterns are regular and predictable in these computing kernels. The memory subsystem design in this thesis is optimized for the predictable computing kernels of streaming signal processing.

Investigate parallel processor architectures. Different parallel processor architectures are investigated in this thesis. It studied different levels of parallelism implemented by various parallel processors, including the instruction level, the data level, and the task level parallelism. The investigation identifies the performance obstacles related to memory access and data transfer in parallel computing, such as the streaming data transfer, the parallel data access overhead for 
parallel data path, the DRAM memory access efficiency, and the memory coherency overhead and the multicore control overheads. The memory subsystem design in this thesis is optimized to reduce the impact of the performance obstacles.

Define stream oriented multicore computing paradigm. This thesis proposes a stream oriented multicore computing paradigm. The parallel computing tasks are triggered and synchronized by data streams. A light weight RISC controller performs the central management of data streams. Each computing kernel on one DSP core works as a stream consumer and a stream provider. The DSP kernel is programmed to include both communication functions and computing functions. The communication functions move data streams between the network interface and the local memory. The computing functions apply computation algorithms on local data. The stream oriented computing paradigm simplifies the parallel software and hardware design. It reduces control overhead by starting computing as soon as the data stream arrives. The centralized software stream manager eliminates the memory coherence problem on the multcore DSP.

Memory subsystem architecture design and implementation. This thesis contributes to the ePUMA project in the hardware implementation of the multicore DSP. It designs the memory subsystem architecture and implements the hardware to realize the stream oriented parallel computing paradigm. The hardware design includes the implementation of the Ring and Star stream networks, a serial bus for message passing, the central DMA controller, and the DSP core's local parallel memory. The design is implemented in Verilog and verified in simulation by running compiled software code. The design is synthesized using ST Microelectronics 65nm CMOS technology to evaluate the performance and area.

Simulation model and software toolchain implementation. Another contribution of this thesis is the modeling of the memory subsystem 
and the master controller. A cycle accurate simulator is developed to evaluate the architecture design at the early design phase. The software model is also used as the reference for the hardware implementation. Contributions in the development of software toolchain include the implementation of software library functions to control the multicore computing and the memory subsystem, and a multitasking framework ported to the master controller.

Application analysis and implementation. A number of DSP applications are analyzed and implemented on the multicore DSP platfor$\mathrm{m}$ for evaluation. The applications include a Motion JPEG encoder [20], an MPEG-2 decoder [21], and an LTE downlink baseband processing unit. Other DSP kernels like matrix multiplication [22] and convolutional decoder [23] are also mapped to the parallel DSP.

\subsection{Thesis Organization}

The thesis consists of three parts. The first part introduces the background. Chapter 2 gives an overview of the computational characteristics of streaming applications. Chapter 3 investigates different parallel processor architectures and multicore architectures. The performance obstacles are analyzed and existing architecture examples are introduced. In chapter 4 the theory of parallel memory architecture and conflict free access are introduced.

The second part describes ePUMA multicore DSP architecture and its memory subsystem design. Chapter 5 gives an overview of the multicore DSP system. Chapter 6 depicts the details of the memory subsystem architecture. Chapter 7 introduces the programming model and the tools and flow to build ePUMA software.

The third part of this thesis is about results and conclusion. In Chapter 8 , software implementation of applications on the multicore platform is described. The performance and area evaluation results are collected in chapter 9. Chapter 10 concludes the thesis and discusses the future 
work of the on-going ePUMA project. 


\section{Chapter 2 \\ High Performance \\ Streaming Computing}

The contribution of investigating the computing characteristics of streaming computing is described in detail in this chapter.

Developing a computing platform requires a proper appreciation of the computational characteristics of the target applications and algorithms. Streaming signal processing in multimedia, graphics, and communication applications requires computing throughput ranging from $0.1 \mathrm{GOPS}$ to 100 GOPS [1]. There is always a growing trend in the performance requirement in these application fields. Most of these applications must meet real-time deadlines. Aspects such as data type, control flow, data movement and so on are important in understanding applications. In this section, the computing characteristics of streaming applications will be summarized first. Then we focus on the computing kernels and data access to further explore the computing characteristics.

\subsection{Computing Characteristics}

The computing characteristics of the target applications are listed in Table 2.1. The streaming applications have a common task of processing a 


\begin{tabular}{|c|c|}
\hline Characteristic & Description \\
\hline Throughput & $0.1 \sim 100$ GOPS \\
\hline Latency & short latency $(0.1 \mathrm{~ms} \sim 1 \mathrm{~s})$ \\
\hline Input & Continuous and periodic streaming data input \\
\hline Data type & Fixed point (less than single precision) \\
\hline Data size & $\begin{array}{l}\text { Large volume of data, stored in arrays or simple } \\
\text { data structure (e.g. RGB structure for image data) }\end{array}$ \\
\hline \multirow[t]{2}{*}{ Control flow } & $\begin{array}{l}\text { Data flow based signal processing with multiple } \\
\text { computing stages }\end{array}$ \\
\hline & Data independent control flow \\
\hline Computation & $\begin{array}{l}\text { Inner most loop consists of combination of basic } \\
\text { computing operations such as MAC and vector } \\
\text { operations }\end{array}$ \\
\hline \multirow[t]{4}{*}{ Data access } & Data are accessed only few times in computing \\
\hline & Data access is predictable and data-independent \\
\hline & Data access patterns include sequential, stride \\
\hline & based, or predefined index based \\
\hline
\end{tabular}

Table 2.1: Computing characteristics of streaming applications 
large volume of input data to extract information. For example the main task of baseband signal processing in wireless communication is to remove noise and interferences on the useful information bits. Such applications are characterized by high computational throughput, but require simple control flow and regular data accesses. The latency is the time between the arrival of input data and the output of corresponding computing results. It includes computing time and data access overhead. The latency in our target applications is very short with respect to the large data volume and high computation load. For example, the LTE baseband signal processing requires a computing latency of $1 \mathrm{~ms}$ to process one subframe. Within this $1 \mathrm{~ms}$, the baseband processor needs to do a large number of filter and matrix operations for channel estimation and equalization [24].

The input data of streaming applications are continuous data stored in memory or received from analog sensors. The data type is fixed point, with a precision of 16 bits or less. The signal processing is triggered periodically by external events when new data arrives. The data are stored using regular structures such as vectors and multi-dimensional arrays.

The control flow is simple and data independent. The target applications usually consists of a combination of multiple computing stages. Each stage contains iterative execution of basic arithmetic operations. The arithmetic operations at the inner-most loop form the computing kernels. These kernels consume most of the execution time. The programmable DSP solutions usually optimize the Instruction Set Architecture (ISA) and microarchitecture design to accelerate the computing kernels.

Most of the computation kernels in streaming signal processing require regular and predictable data accesses. The access patterns are data independent, meaning that the data allocation in memory can be determined before execution and it does not depend on the value of the data. This is important for memory subsystem design that relies on predictable data accesses to optimize data movements to reduce the chance of conflicts and hide communication overheads behind computing. The 
common access patterns include sequential, stride based and predefined index based.

In streaming computing, the input data is only accessed a few times by one kernel. The arithmetic operations applied to the input data can be simple single step operations, such as MAC, or a complex mix of multiple operations such as $\mathrm{QR}$ decomposition. The computing kernel updates the input data or generates new data packets for processing at the next computing stage.

Understanding the caching characteristics of streaming applications is important for memory subsystem design. Traditional data caching does not work well for streaming data. Cache architecture is effective only when the data stored in cache is accessed very often. But in streaming applications, the input data is only accessed a few times for computing and transformed into a new data stream; after that the original data is discarded. Low access rate streaming applications cannot benefit from cache memory very much. Scratch Pad Memory (SPM) or Tightly Coupled Memory (TCM), which works as high speed on-chip data buffer, is suitable for streaming applications. This approach requires software function to move data to the SPM for computing. For predictable algorithms, it is beneficial to explicitly control the data movements to overlap with computing so that memory access overhead is reduced.

\subsection{Computation Kernels}

Table 2.2 lists the most common computation kernels in streaming signal processing. These kernels belong to five categories of computations: filter, transform, vector operation, matrix operation, and arithmetic function solver. The computation kernels are found in the inner most loop of application algorithms. They are the most computationally intensive part in streaming applications. In some kernels numerical functions are used such as square root $(\sqrt{x})$, reciprocal $\left(\frac{1}{x}\right)$, and trigonometric functions ( $\sin , \cos )$. Computing these functions usually requires optimized subroutines or specific hardware. An alternative solution is to use a SIMD ex- 


\begin{tabular}{ll}
\hline \hline Category & Computation kernel \\
\hline Filters & FIR filter \\
& IIR filter \\
& Interpolation filter \\
& Decimation filter \\
Transform & FFT $/$ IFFT \\
& DCT/IDCT \\
Vector & Add/Sub \\
& Multiplication \\
& Min/Max \\
& Sum \\
& Sort \\
Matrix & Multiplication \\
& Transpose \\
& Inverse \\
& QR/LU decomposition \\
& SVD \\
$\frac{1}{x}, \frac{1}{\sqrt{x}}$, sin, cos,... \\
\hline \hline
\end{tabular}

Table 2.2: Typical computation kernels in streaming signal processing

tension to perform fast approximation of these numerical functions [25]. This solution is based on Taylor series expansion and has the advantage of fixed computing latency, which is preferred by streaming applications with strict computing deadline. The vector and matrix operations are usually called in nested loops to repetitively execute on a large number of input data sets, which shows a high degree of data level parallelism.

Since most streaming computing systems compute on fixed point input data, data precision and dynamic range are major issues, especially when the algorithm goes through a large number of iterations. For example, a radix-2 2048 point FFT requires 11 layers of computation. The dynamic range of the produced data increases after every layer. The precision decreases if a constant scaling is applied after each FFT layer. Block floating point can be used to increase the dynamic range, where a block 
of data share the same exponent. Block floating point is an intermediate solution between fixed point and floating point.

The kernels contain both real number computations and complex number computations. Most wireless baseband signal processing algorithms require complex data with a real part and an imaginary part. Some DSP processors have instructions to support complex arithmetics. However, input data allocation can affect the computing performance dramatically if data reorganization is required for computing. For example, suppose a vector of complex numbers is stored as a data block of all real part numbers followed by a data block of all imaginary part numbers. The access of one complex number would then require two reads from two memory regions. Data access overhead can thus limit the overall computing performance.

Although the memory accesses are regular and predictable in streaming computing, some kernels require special address calculation for vector or matrix indexing. Those address calculations are executed in the inner most loop of the algorithms, therefore hardware acceleration for address generation can effectively improve the computing performance. For example, in FFT, bit-reversed addressing is required to access a data pair to perform the butterfly operation. Most DSP processors have introduced the bit-reversed addressing mode in their Address Generation Unit (AGU) to accelerate FFT computation. Another kind of special indexing of vector and matrix is defined in application standards. For example, the 4G LTE physical channel standard [26] defines the location of reference signals in the received OFDM symbols as illustrated in Figure 2.1. The allocation differs between different system configurations such as number of antennas, bandwidth, and Cyclic Prefix (CP) size. Channel estimation based on the reference signals needs to extract them from the received data stream. The address computation needs the assistance of a lookup table to store the specific address information. 


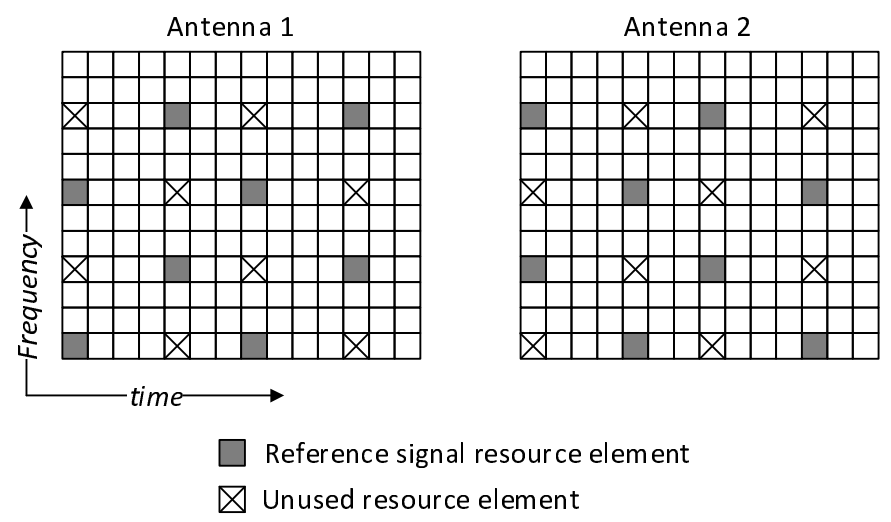

Figure 2.1: Reference signal allocation in LTE for a case of two antennas system

\subsection{Data Flow Based Signal Processing}

Most algorithms of streaming applications can be described as a chain of computing stages. Each stage contains one or multiple computation kernels. The connections between the stages are data streams. Computing result of one stage is passed to the next stage as input. This section will introduce the signal processing flow of three typical streaming applications: the LTE baseband signal processing flow as a communication example; the H.264 codec to demonstrate a multimedia application; and a rendering pipeline for computer graphics applications.

\subsubsection{LTE Uplink Baseband Signal Processing}

A simplified data processing flow in the physical layer of LTE uplink is shown in Figure 2.2. The heavy computing load at a LTE basestation comes from the multiuser signal processing. LTE physical layer uses the Single Carrier-Frequency Division Multiple Access (SC-FDMA) as the basic transmission scheme to handle multiuser communications in the uplink [26]. The computing blocks in the data flow include the preprocessing of removing cyclic prefix, the input FFT, and for each user, it performs synchronization, channel estimation, channel equalization, 


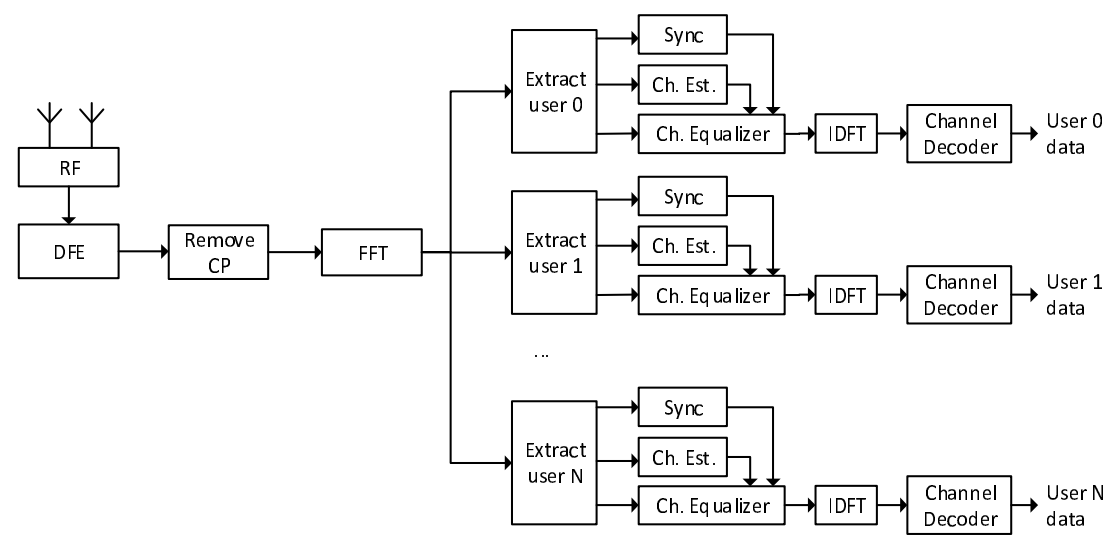

Figure 2.2: LTE uplink signal processing flow

IDFT computing and channel decoding. Most of the computing blocks, for example the FFT and channel equalization, are suitable for ASIP processors, which use parallel datapath and special addressing modes to achieve high performance. The channel decoding block is usually implemented by an ASIC accelerator, because of the high computing throughput requirement such as a Turbo decoder [27].

\subsubsection{H.264 Decoder}

Figure 2.3 shows the data flow of a H.264 [28] video decoder. It contains both bit processing and pixel computing. The first two stages, the bitstream parser and the CABAC/CAVLC decoder, are bit level processing. General purpose processors are not efficient in processing bit streams. It is common to use customized instructions or hardware accelerators to do bit level processing. The remaining blocks in the data flow include data intensive matrix operations and transform computation. For example, the motion compensation process performs a matrix addition of the predicted data and the decompressed residual and stores the result into the frame buffer for the decoded picture. 


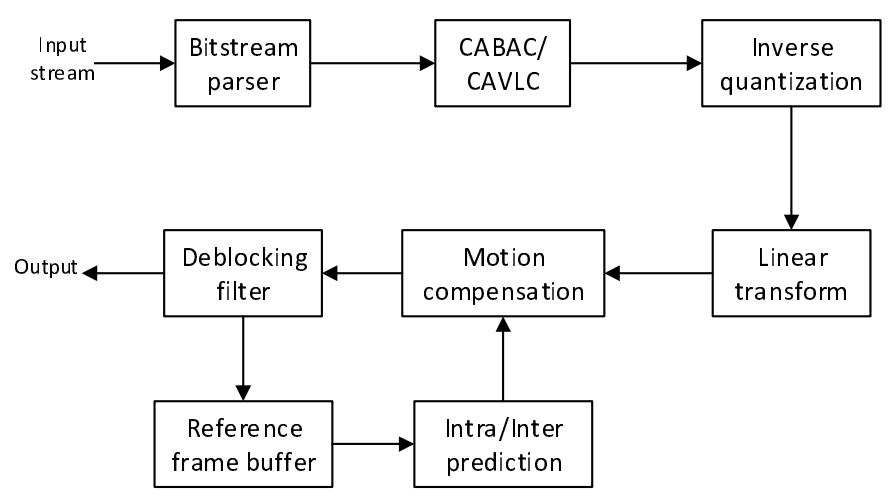

Figure 2.3: Signal processing flow for H.264 video decoder

\subsubsection{D Rendering Pipeline}

Figure 2.4 shows the OpenGL 3D rendering pipeline [29]. The computing stages in this pipleline mostly operate on floating point data. The communication between functional blocks are streams of predefined data structures, for example vertices of $3 \mathrm{D}$ coordinates and primitives. The vertex processing performs vertex transformations using vector-matrix multiplications. The primitive assembly converts vertices to shape primitives according to the required drawing mode (a command received from OpenGL API). This stage contains only scalar operations. The clipping and culling modifies the primitives to screen borders. Data parallel processing is possible in this computing stage. The remaining stages of this flow are pixel processing. In the rasterization stage the primitives are converted into pixel coordinates. The fragment processing stage modifies the pixels to draw according to the color and other attributes of the pixels. The last stage of this pipeline is to write the pixels to the frame buffer. The video display controller periodically reads the framebuffer and outputs the refreshed picture to the monitor.

The programmable hardware for computer graphics uses a parallel architecture called SIMT, Single Instruction Multiple Threads [30]. Compared to the Cray style vector machine's SIMD architecture [31], which uses vector instructions to operate on multiple data points, the SIMT ar- 


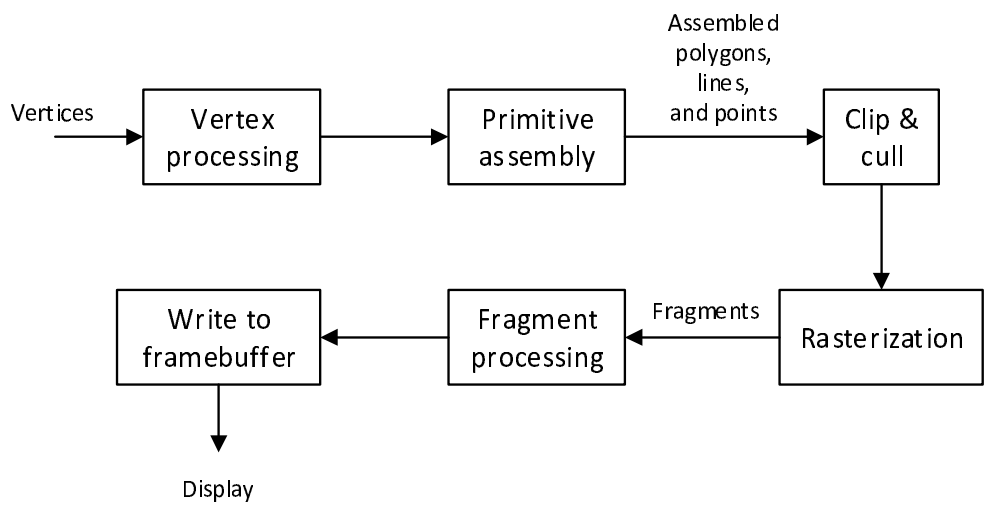

Figure 2.4: OpenGL 3D rendering pipeline

chitecture can be interpreted as multiple independent scalar threads. 


\section{Chapter 3}

\section{Parallel Processor}

\section{Architectures}

The contribution of investigating the parallel processor architectures is described in detail in this chapter.

\subsection{Introduction}

Streaming signal processing requires great computing power to process large volumes of data using complex algorithms. To accomplish this task, programmable solutions need to explore all possibilities for parallel execution. There are different forms of parallelism implemented by different processor architectures, including Instruction Level Parallelism (ILP), Data Level Parallelism (DLP), and Task Level Parallelism (TLP). ILP is used by superscalar [32] and VLIW [33] processor architectures. DLP is used in most SIMD [13][34] and vector processors [31] to operate on multiple data by single instruction. Multiprocessor architecture [8] for TLP is used in most System-on-chip solutions. The following sections will introduce these parallel architectures and discuss their advantages and potential performance obstacles. 


\subsection{Instruction Level Parallel Execution}

A single instruction flow processor implements multiple execution units to increase its computing power by executing multiple instructions in parallel. Each execution unit can handle one type of operation, for example ALU unit, load-store unit, and floating point unit. There are two different approaches in ILP to decide which instructions are executed in parallel: hardware and software. The processor architectures implementing these two approaches are known as Superscalar and VLIW.

\subsubsection{Superscalar}

The hardware approach relies on hardware logic to detect instruction dependencies and reschedule the instructions to execute on different hardware units in parallel. This type of machine is called Superscalar. It is a common architecture used by general purpose CPUs such as the Intel Pentium processor. A Superscalar processor executes a sequential instruction stream, the parallelization of instructions is done by hardware. The performance boost comes at the cost of extra hardware, which also increases the processor core's power consumption. Superscalar is a suitable ILP architecture for general purpose computing such as the application processor of a mobile platform. But it is not a power efficient architecture for streaming signal processing, because the control complexity in streaming signal processing is not high, only regular control flow is required.

\subsubsection{VLIW}

The software approach relies on the software programmer or compiler tools to explicitly define the instruction groups to be executed in parallel. Processors which implement this approach are called VLIW processors. VLIW is a popular architecture among DSP processors, for example TI C6000 [4] and Tensilica DSPs [35]. Some VLIW processors use a dedicated bit to indicate that the current instruction is executed with the previ- 
ous instruction in the same clock cycle. The assembly program supports such syntax. For example the TI DSP assembly uses the symbol "I I for parallel execution with previous instruction [36]. Multiple instructions are grouped into one long instruction word to be loaded by the VLIW processor.

A VLIW processor simplifies the hardware implementation of an ILP. It does not need hardware logic to do runtime instruction scheduling as in Superscalar processors. The instruction parallelization is done offline at compile time. Therefore VLIW processors have extra demand on compiler design. However, in order to achieve the best computing performance, most of the computation kernels for a VLIW processor are programmed in assembly and provided as library routines. Assembly programming for instruction level parallel execution typically uses techniques such as loop unrolling and software pipelining. The programming complexity is high for VLIW processors.

A VLIW processor has low hardware efficiency. It needs multiple instruction decoders to decode instructions in parallel. VLIW processors usually implement a large register file in order to maximally utilize the multiple execution units during computation and avoid conflicts on register usage. Moreover, the program size of a VLIW processor is normally larger than other architectures due to its wide instruction word and software optimizations for example loop unrolling.

VLIW architectures can achieve high performance in loop executions by loop unrolling and software pipelining. Therefore it is the preferred architecture of DSP processors, where most computation kernels are based on iterations, for example the FIR filter. However, it still mixes control and computing in the long instruction word, when the program executes a branch or encounters a memory stall, the utilization of execution units becomes low. 


\subsection{SIMD Architecture for Data Level Parallelism}

A SIMD architecture executes a single instruction on multiple data points in parallel. For applications dominated by regular vector/matrix operations, a SIMD architecture can provide excellent computing performance and high hardware efficiency. It is widely used for embedded applications in multimedia, graphics, and baseband signal processing of communication. A SIMD extension has been embedded in most standard processor architectures including RISC, Superscalar and VLIW, as an extension to accelerate data intensive computation.

\subsubsection{SIMD Arithmetic Functions}

The arithmetic functions performed by SIMD instructions can vary from simple flat parallel operations to complex fused operations. The complexity of SIMD instructions keeps growing to accelerate various DSP algorithms. This leads to the use of a deep pipeline in most SIMD architectures to support multi-step arithmetic operations.

The most common SIMD computing form is the flat parallel operation, where the same scalar function is applied to multiple data. This basic SIMD operation is implemented by most SIMD architectures. One typical example is the vector add instruction. It takes two vectors as input, adds the corresponding data elements in the two vectors, and writes the result to the output vector. Almost all scalar ALU operations have a corresponding SIMD instruction.

Enhanced SIMD instructions allow more complex operations such as parallel reduction, conditional execution, and data manipulation. Parallel reduction performs the computing by multiple pipeline stages and gets one scalar at output. Typical examples include vector sum, comparison, and MAC operations. Conditional execution is supported by adding one step before the computing stage to test the input data or vector flag and mask the SIMD arithmetic computing. Data manipula- 
tion here means the pre- and post- data manipulation operations such as data type conversion, scaling, saturation, rounding, and etc.. Combining multiple operations in a single SIMD instruction can improve performance by reducing the instruction cycles, especially when the instruction is used in the innermost loop of an iteration. Another benefit of a combined operation is on the saving of intermediate buffers. Some SIMD extensions implement local control logic, e.g. a finite state machine, to process longer vectors than the SIMD width. The SIMD instruction becomes a multi-cycle vector operation.

An important use of SIMD in arithmetic computing is to accelerate the function solver. This is based on the Taylor series approximation. The SIMD datapath is used to accelerate the $x$ power generation. The size of the Taylor series depends on the precision requirement.

\subsubsection{SIMD Data Accesses}

SIMD computing performance depends on the memory subsystem to provide vector data access efficiently. The memory access bottleneck can cause inefficient SIMD computing. In early designs, the SIMD unit as an add-on extension shares registers with other execution units. For example, the MMX SIMD extension [37] shares registers with the floating point unit of the Pentium processor. The conflict with the floating point unit on using registers can reduce the computing efficiency. Later SIMD extensions implement dedicated registers for the SIMD unit [38].

Data manipulation is another important factor that limits SIMD computing performance. Most SIMD architectures have instructions to perform vector permutation [39], for example packing and unpacking, shift and rotation operations. The purpose of these instructions is to reorganize the input vector data to the computing order of a SIMD arithmetic instruction.

The SIMD architecture for DSP usually employs a large on-chip data buffer for SIMD computing. It uses a DMA controller to transfer a block of data between the local buffer and external memory. Existing im- 
plementations of the local data buffer include a multi-port register file, a wide single port SRAM, and a multi-bank memory. The multi-port register file provides high flexibility for irregular data access. It is used by the eLite SIMD processor [40]. The high hardware cost and power consumption limit the size of the register file buffer. A single port memory is simple to implement and has the lowest silicon cost. But it lacks the flexibility in data access; only aligned data access is supported. Unaligned data access and other irregular data access (e.g. stride based) have to be split into multiple accesses and be completed in multi-cycles, which increase memory access latency and affect the SIMD computing performance. A multi-bank memory is widely used as a trade-off between implementation cost and data access flexibility. There are both software and hardware approaches to reduce parallel data access latency on a multi-bank memory. The software solution optimizes the data allocation in the multi-bank memory by compiler techniques [41]. However, since the SIMD compiler itself cannot show enough performance at auto vectorization of a high level language such as $C$, current optimization of SIMD programs still use intrinsic and assembly coding. The hardware approach uses a shuffle network for data shuffling, for example the SODA processor [42]. The aim of the shuffle network design is to minimize the clock cycles that are required to reorganize vector data for computing.

All the aforementioned solutions are based on a fixed input data allocation in the data buffer. The data is usually stored in a sequential order when they are transferred through the DMA controller. For a multibank memory, more data access flexibility can be obtained by reorganizing the input data allocation to avoid a bank conflict for certain parallel access patterns. The memory architecture design is described in [17]. Predictable computing algorithms can benefit from this memory subsystem and achieve high computing efficiency. This approach is one of the essential technologies of the ePUMA processor. 


\subsubsection{SWAR Extension vs. SIMD Engine}

There are mainly two classes of SIMD architecture implementations, the SWAR extension and the SIMD engine. SWAR stands for SIMD With A Register, it is widely used as an instruction set extension in general purpose processors. For example the Intel Pentium desktop processor's SSE extension [43] and the ARM embedded processor's NEON extension [34][44]. It uses a group of registers and a set of SIMD instructions to enable data parallel execution. The SIMD instructions are a part of the CPU's instruction set, it eases the software development by using only one instruction stream and a compiling tool. Computing kernels accelerated by the SIMD datapath are usually programmed using inline assembly or intrinsics. Most SWAR extensions only provide simple and regular SIMD arithmetic functions because of the limited control over the data path encoded in the short instruction word.

Another type of SIMD implementation can be found in dedicated SIMD engines which are mostly used for digital signal processing. Examples of such engines include vector processors [31] and DSP processors [5] [14], where the SIMD datapath forms the computing core. The advantage of the SIMD engine is that it can support more complex SIMD operations and flexible data access formats than SWAR extensions. The instruction set can be customized for a specific application and the memory subsystem can be optimized for fast parallel data access.

\subsection{Chip Multiprocessor}

To meet the ever-increasing demand on computing power, modern systemon-chip solutions integrate multiple processing cores to do task level parallel execution. The multicore solution becomes popular when the single core reaches the frequency limit. A single core processor relies on custom design to achieve very high operating frequency, which requires a large design effort. Moreover, the power consumption grows non-linearly with the increase of frequency. Processors running at high 


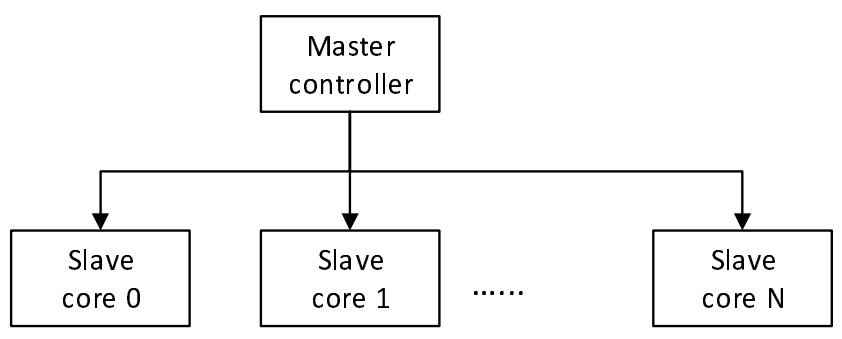

Figure 3.1: Multicore master-slave computing model

frequency are not power efficient. Multicore solutions can meet the performance requirement at a lower operating frequency and the processor cores in a multicore system do not need custom design to achieve ultimate speed. However, the design of a multiprocessor architecture needs to consider other issues such as the parallel computing model, the memory subsystem, and the interconnection architecture, in order to reduce the control and communication overheads.

\subsubsection{Multicore Computing Model}

The computing model defines the collaboration between processor cores in a multicore system. The hardware design, memory subsystem and interconnection network, should support the computing models at high execution efficiency. Most signal processing algorithms are designed for sequential execution on a single core. The introduction of multicore processors brings a new challenge: to map the application to a multicore processor [45]. The software developer must handle task distribution on multiple execution units and manage data communication between tasks. There are mainly three computing models in multicore parallel processing; the master-slave model, the data-flow model, and the mixed model of these two.

The master-slave model uses one master core for centralized control, and other slave cores to execute subtasks, as illustrated in Figure 3.1. The master core has less computing load, its major task is to run the control code and balance the computing load on slave cores. It also prepares data 


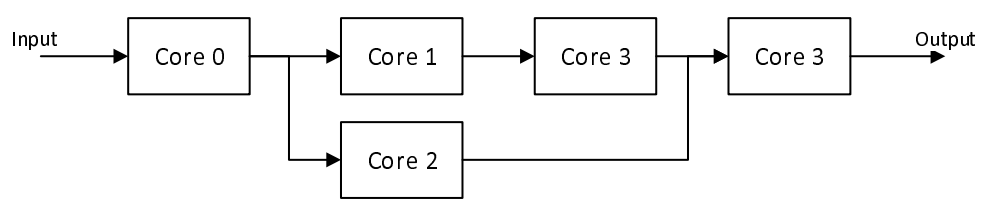

Figure 3.2: Multicore data-flow computing model

for computing on slave cores. The subtasks are independent in execution, no communication are required between slave cores. The master-slave model is suitable for a homogeneous multicore platform which handles dynamic tasks. The master core often runs an operating system to schedule randomly activated tasks based on the task's computing requirement and the slave cores' availability. A typical application is the multi-user signal processing in the uplink of wireless communication [46], where the number of users connected to the basestation and the bandwidth assigned to each user change dynamically. The master core is responsible for dynamic scheduling of single user signal processing to a slave core. It also extracts the user data and transfers the data to the slave core for computing.

The data flow model performs the computing in the way of a signal processing pipeline, as illustrated in Figure 3.2. Each core is assigned one or multiple computing tasks, it receives data from previous core(s) and processes the data using various algorithms. The output data are transferred to another core for further computing. The task execution is triggered by input data. The initial core often uses an external data interface to receive input data. The data flow model is suitable for statically scheduled multicore parallel processing with high computing load. It can use heterogeneous processing cores to accelerate specific computing tasks. The architecture design challenge to support a data flow computing model is on the inter-processor communication. Firstly, the network should support concurrent data links between processing cores. A traditional shared bus allows only one data transfer at a moment, which can hardly meet the communication throughput requirement. Secondly, inter-processor data transfer is usually burst based. It moves a block of 


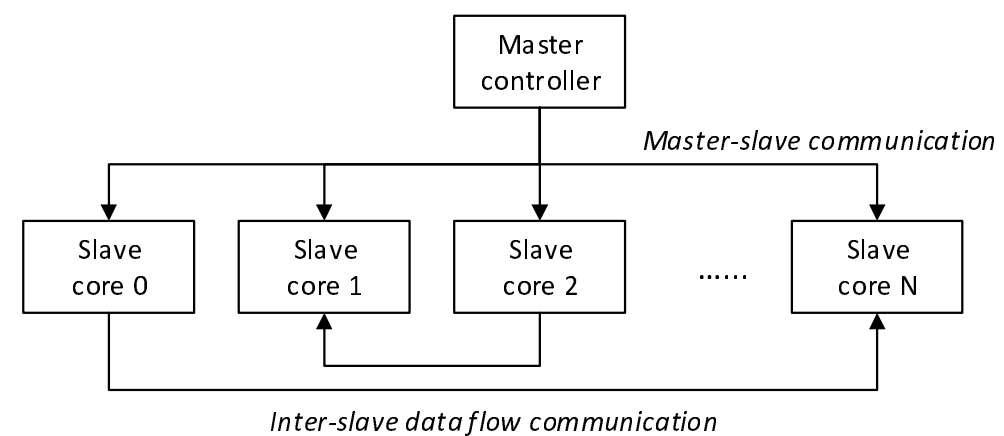

Figure 3.3: Multicore mixed master-slave and data-flow computing model

data from one computing buffer to another buffer. The access patterns are regular. Network design can be optimized for streaming data transfer, for example, to increase the burst length and change the arbitration granularity for stream requests. Low latency inter-processor communication is critical in data flow computing model. Most multicore implementations use ping-pong buffers to hide the communication latency.

The third computing model is a combination of the master-slave model and the data flow model. It is used in complex applications which requires both top flow control and high throughput data communication between slave processors. The centralized control has the advantage at handling common resources such as shared memory, DMA controller, and external data interface, to avoid conflicts and schedule data access for best performance. The concurrent task execution on slave cores takes the advantage of each core's computing power. When data transfer is required between slave cores, it doesn't need to go through the master core to exchange data, the on-chip network can support the inter-processor communication efficiently.

\subsubsection{Shared Memory vs. Distributed Memory}

The memory architecture determines how data are transferred from one core to another. There are mainly two types of memory architectures in 
multicore processors: shared memory and distributed memory.

In a shared memory architecture, all memories are mapped to the global memory space. All cores have the right to access all memories. A data transfer is done by the sender who writes data to a memory buffer and notifies the receiver. The receiver then reads data from the shared buffer. Memory coherence is important in shared memory architecture. Frequent notifications can happen between processors to communicate the ownership of shared data. Extra hardware in the interconnection network design is also necessary to support atomic access to manage race conditions on the shared data. From a software programmer's point of view, shared memory is preferred because it provides a flat and unified view of all memory data. But shared memory architecture usually limits the computing performance by its low memory access speed. Because memory is not tightly connected to the processing core, one memory access might need to go through bus, memory arbiter, and memory controller to complete, which means a long access latency. To hide the access latency, some cores use cache as local buffer. This will increase the design complexity to handle cache coherency.

Distributed memory architectures implement dedicated memory for each core. The private memory is tightly connected to the core, which can have fast access to the local data. The computation task only applies to the private memory to take the advantage of high access speed. Data exchange with other cores requires explicit communication functions such as DMA, which forces the programmer to handle data movements in a distributed memory system. Inter-processor communication latency is the major performance bottleneck. Most distributed memory architectures use ping-pong buffering to hide the communication latency. In contrast to shared memory architecture, it is easier to maintain memory coherency in distributed memory system, because data in private memory are not shared by multiple cores, and remote memory access needs to notify the remote core before data transfer. 


\subsubsection{Interconnection Architecture}

The hardware blocks in a multi-core system need to communicate with each other during application execution. The on-chip interconnection network is responsible for the communication of data streams. The multicore architecture contains various data traffics with different communication requirements. For example, inter-processor notifications require a short message (a few bytes) to be sent within a short time. Streaming applications need to transfer a large chunk of data at a high bandwidth. The interconnection architecture is responsible for guaranteeing the communication data to be correctly and reliably transferred on the chip. Various types of interconnection architectures can be used which can offer different performance and also have different hardware complexity. High performance networks usually require complex hardware and a high silicon cost.

A shared bus is the most basic on-chip communication architecture. It consists of a set of parallel wires, including a data bus, an address bus, and a control bus. The communication resource is shared by the hardware modules connected to the bus, meaning that at one moment only one module can access the bus to transfer data. A hardware block which can initialize data transfer is called a bus master, for example a processor core. A hardware block which responds to data access requests is called a bus slave, for example an external memory controller. The bus master starts a data transfer by sending a request to the bus arbiter, which manages the shared resources. The data can be transferred when the master is granted the control of the bus. A shared bus doesn't support concurrent data transfer, so that its achievable bandwidth is limited. It can not be used as a high performance interconnection network. However, for its low hardware complexity, a shared bus is commonly used as a peripheral bus to connect low speed IO devices, such as UART and LED displays.

The demand for a high performance interconnection network drives the development of bus architectures to increase communication parallelism and bus utilization. A multi-layer bus or a crossbar architecture 
is implemented to support concurrent data transfers between different source-destination pairs [47]. Other topologies like ring bus [48] and reduced crossbar [49] are design tradeoffs between hardware complexity and performance. A hierarchical bus connects multiple subsystems by bus bridges, each subsystem uses a local bus to connect components. Parallel communications are allowed within different subsystems. The data transfer protocol has been upgraded in advanced bus architectures to save bus idle time in arbitration and transfer, and thus to increase bus utilization and improve performance. Examples of such technologies include pipelined transfer, burst transfer, split transfer, and out-of-order transfer [47].

The emergence of network-on-chip (NoC) architectures [50] over the past few years poses a new approach for multicore interconnection. The NoC architecture uses packet based data transfer via a network consisting of routers and physical data links. The packet routing and arbitration on NoC only evaluate the packet header, which contains address information. During transfer, the data payload does not need to be examined. This simplifies the hardware complexity of the control logic. Other advantages of a NoC architecture include parallelism and scalability. Different physical links can transfer different data packets simultaneously. The NoC architecture is easier to scale than conventional bus architectures since it separates the physical layer and the transport layer. The designer can choose custom network topology which offers better scalability. Packet buffers are the major hardware cost in NoC architectures. Circuit switch NoCs require less buffers than packet switch networks, and it is also suitable for streaming applications with predictable data communications. The circuit switch $\mathrm{NoC}$ evaluates the packet header only at the network interface, then it establishes a path from the source to the destination before data transfer. This network path is locked and only serves one data communication task until the transfer finishes. Packet switching usually has no fixed path for a data packet. It examines the packet header at the routers and determine the next route based on the current network status. It is more suitable for random and unpredictable 
data traffics. Problems with NoC architectures include the overhead for small data packets and the extra setup latency, especially when the communication path goes through many routers.

Most of the aforementioned interconnection architectures are used in shared memory system, where each memory or control register is assigned a globally unique address. The network uses the address information to transfer data. There exists a special class of communication which transfers data to a destination that is not memory mapped, such as video output, radio transmitter, and data exchange between private memories in distributed memory systems. A common solution is to create a custom ad-hoc connection for this type of data transfer. The AXI-4 Stream protocol [51] provides a standard for this kind of stream data transfer. Its special features include unlimited burst length and non-address transfer. The AXI-4 Stream is build upon the normal AXI standard and reuse the handshake protocol of the AXI write channel. The Ring network in ePUMA is also a non-address stream network. It is designed to support stream data transfer between private memories of SIMD co-processors. The Ring network is similar to the AXI-4 Stream on the features such as unlimited stream length and non-address network link. Additionally, it supports stream broadcasting and scalable physical implementation which allows a design trade-off between performance and silicon cost. For applications that require high concurrent inter-processor communication bandwidth, more physical links can be implemented between processor cores to increase the performance.

\subsection{Performance Obstacles}

This section identifies the major performance obstacles in the parallel architectures introduced in this chapter. In general, most of the performance obstacles are related to memory access and data transfer. Avoiding these obstacles and minimizing their effects are the major goals when designing the memory subsystem of ePUMA. 


\subsubsection{Streaming Data Movement}

Streaming applications usually requires a large amount of data to be transferred between processing cores, the communication latency can not be ignored in high performance signal processing and becomes the major overhead. Most streaming computing platforms use a DMA controller to move a bulk of data. The DMA controller transfers data independently of the processor core. It can thus alleviate the processor load on long time read and write operations. The processor can have more time to perform computations. The processor core initiates a DMA transfer by configuring a number of parameters including size of transfer, selection of source and destination, and memory addressing patterns. The communication latency includes the initialization time and the transfer time of data payload.

DMA initialization latency is mainly determined by the addressing complexity. For a simple continuous data block, only the start address and the size parameter are required. Therefore the initialization can be done with short latency. For complex addressing patterns, a lookup table containing addresses for every single data might need to be configured, which requires a significant time for initialization. In predictable streaming computing, various techniques can be used to save the task configuration time [52]. Those complex addressing patterns can be pre-stored in the descriptor memory as off-line data to save DMA initialization time.

The communication latency for a data payload transfer is determined by the amount of data and the interconnection architecture. A simple and effective approach to reduce communication latency is to increase the data bus width. For example, a common high bandwidth bus uses a 128 bit or a 256 bit data bus. The bus width is chosen to match the data throughput of the local memory subsystem. Otherwise data packing will be required, and can not match the network bandwidth. Most bus systems cannot fully utilize all bus cycles on a data transfer. Burst transfer mode allows a data sequence to be transferred on the bus at a high bandwidth of one valid data beat per cycle. However, the maximum burst 
length is much less than the required stream size in signal processing. A streaming bus and a $\mathrm{NoC}$ architecture have no limitation on maximum packet size, for example the ARM AXI-4 Stream. They are suitable for streaming data transfer.

Another significant performance penalty is on data broadcasting, where the same data is accessed by multiple processors for computing. Data broadcasting is a common communication model in multicore parallel computing when one computing task is divided into subtasks and distributed to multiple processor cores. For example in parallel computing of a large matrix multiplication [22], one input matrix is divided into several sub-matrices and equally distributed to multiple cores, the other input matrix is shared by all the cores for their local matrix multiplication. Redundant data transfer of the common matrix increases the communication latency, especially for parallel computing using a large number of cores. Communication overhead can be dramatically reduced if data broadcasting of the common matrix is supported. A typical interconnection network only supports data transfers between two components, a data source and a destination. In a shared memory architecture, it is hard to broadcast data to multiple destinations by a single physical data transfer because only one address information is carried with the data.

\subsubsection{Parallel Data Access for a SIMD Datapath}

Parallel data access latency is the major performance obstacle of a SIMD architecture. A SIMD processor offers high computing capability by the parallel datapath, which can process multiple input data in one cycle. The computing operands can be a register or a memory in different architectures.

Some SIMD processors use the load-store architecture which only allows arithmetic operations to be performed on registers. Memory data are accessed by load and store instructions. Such an architecture requires extra instructions or cycles to move data between a memory and a register file for computing. In the inner most loop of computation kernels, 
the load or store instructions can dramatically decrease the performance in single issue processors. For example, one SIMD arithmetic instruction with two input operands and one output would require two load instructions and one store instruction for memory accesses. The data access overhead is $300 \%$ (assuming that the data is in local memory). Some VLIW+SIMD combined architectures hide the data access overhead by running a load store instruction in parallel with a SIMD computing instruction. Such architectures have the common problems of VLIW processors including a large register file and a large program size.

Load-store architectures use wide registers to provide parallel data for SIMD computing. Data shuffle operations on these special registers are very common in SIMD kernel programs since the computing order are usually different from the data order in the memory. The data shuffle operations can take up to $80 \%$ of the execution time of a SIMD program, where only $20 \%$ is useful computation time [15].

The register memory architecture allows to use memory operands in arithmetic operations, which avoids the register load and store overhead. The performance of this architecture is high only when the data access latency is short. Long latency memory access would stall the processor until the data arrives. Usually in such architectures data are preloaded to local memory before they are accessed for computing, for example to data cache or SPM. The disadvantage of using a register memory architecture is that the local memory cannot provide enough flexibility in parallel memory access, only continuous data in the memory is accessed as a computing operand. Because of the memory access limitation, most of such architectures use narrow SIMD width, for example vectors of two or four elements.

\subsubsection{DRAM Memory Bandwidth Efficiency}

Double data rate (DDR) SDRAM is commonly used in embedded systems when large volume data storage is needed. It has the advantage of low hardware cost per bit. The data access latency and bandwidth of 
DDR memory are critical to system performance. In most SoC systems, the DDR memory is shared by multiple on-chip processing units at run time, for example to store program data for a micro controller, frame buffers for video codec, and data buffers for communication stack. The access requests to the DDR memory from different processing units may happen simultaneously, randomly and target different memory addresses. The memory organization of a DDR memory makes the data access protocol complex, for example the burst based data access and the requirements on refresh and pre-charge operations. Therefore the random read and write accesses may dramatically decrease DDR memory performance. Another factor to be considered is that the DDR memory usually serves various data communications for multiple on-chip processors or IP blocks in a SoC design, with each one having different latency and bandwidth requirements.

Various optimization approaches have been used in DDR memory controller design to improve performance. The most common one is to reorder the command queue by using one read command buffer and one write command buffer. The memory controller selects commands to execute from these two buffers in an order with minimum latency. Because the switching between read and write accesses will introduce longer delay in a DDR control process, a typical solution is to reorder the command queue to execute several reads followed by several writes. For data access requests with different latency requirements, the command queue can be associated with a priority value and the memory controller will serve high priority accesses first.

Saving external memory bandwidth is a major concern while designing a multicore processor for an embedded system, because the external data access is slow and cost a lot of power. The commodity DRAM devices have different variants including normal DRAMs such as DDR/DDR2/DDR3/LPDDR and high performance DRAMs such as RLDRAM [53] and FCRAM [54]. A generic DRAM model can be abstracted for measuring the performance of different generations of DRAM devices [55], which means an optimization strategy for one type of DRAM can 
usually improve performance in other DRAM based systems.

\subsubsection{Memory Coherency Overhead}

Memory coherency is a common issue in multicore systems when multiple copies of shared data are loaded and modified in different memory entities, for example caches and SPMs. The potential problem is on updating data in the correct order for all the data copies in the system, and to make sure all processor cores access the current data instead of outdated data when they operate on their local memory. Hardware solutions are widely used to address the cache coherency in a shared memory system, which have mainly two approaches: the bus snooping architecture and the directory based cache coherency protocol [56]. In a bus snooping architecture, all bus masters listen to the bus transactions and change the state of the local copy. For example, when the bus monitor detects a write access for which it has a local copy of the data, it will mark the data as dirty or invalid. A bus snooping architecture has limitations on scalability because the snooping traffic is proportional to $N^{2}$, where $N$ is the number of processors.

Another hardware approach is the directory based cache coherency protocol. It uses a common memory space to store the directory, which contains the information of where the cached line within the system is held. The coherency protocol requires all memory accesses to first lookup the directory to get where the data is cached and then synchronize data in the listed caches. It improves the scalability by filtering out the unnecessary coherency traffic. However, since every access starts from consulting the directory, the access latency increases.

Pure hardware support for automated cache coherency is not an optimal solution for an embedded DSP system dominated by predictable computation, because of the extra power consumption and latency overhead. Most memory subsystem designs for DSP use software management to explicitly control data movements in the memory hierarchy. The software developer controls when and if local data are copied into dif- 
ferent memories. This is the way to achieve fast execution at low power for applications with predictability and determinism. The software approach is the only solution for a SPM-based multicore architecture to maintain memory coherency. Those operations add to software complexity and cost processor time. A parallel programming model and hardware support to hide data communication latency are important.

\subsubsection{Control Overhead}

The control overhead in a multicore system includes the execution time of software routines for synchronization, short message passing, and subsystem configuration. Complex software with high overhead will not only reduce system performance but also affect software programmability. For parallel processing on a multicore system, the computing job is divided into multiple sub-tasks which are distributed to several parallel processing units. Each task needs to synchronize with one or more other tasks during computing. The synchronization in software is usually done by register pooling or interrupts. Both approaches have software overhead up to tens of processor cycles. Software complexity will increase if all synchronization tasks are done by inter-processor interrupts, because the interrupt routines are outside the program flow. Moreover, the interrupt processing latency increases when the number of cores is large. For predictable computing, it is better to use register pooling or mailbox mechanism than interrupts for inter-processor synchronization.

Short message communication is used to pass function parameters or perform synchronization between processors. It can use special hardware for example a mailbox or use a shared memory space with mutex access support to pass messages. However, the message passing usually requires a handshaking protocol between the initiator and the receiver which involves long latency. Another potential problem is the message communication conflicts with streaming data transfer. A common onchip interconnection architecture uses the same shared bus to support all kinds of communications including short message passing and stream 
data transfer. The short message communication has less data but requires low latency, while the stream data transfer with a large data volume requires high communication bandwidth. Merging this two kinds of communication in the same physical network will have negative impact on the performance.

A subsystem configuration will cost processor time especially when there are many configuration parameters for example the DMA controller. A typical configuration of a DMA task requires the parameters for source and destination address calculation, data types, and triggering events. When the configuration latency is long, it is beneficial to use multiple buffers to store parameters, so that when the DMA engine is working on the current task, the processor can write a configuration of the next task to hide the configuration overhead.

\subsection{Programmable Architectures for Streaming Applications}

This section introduces two examples of programmable parallel architectures for streaming applications. Those processor architectures are designed for applications that have similar computing characteristics as the target applications of ePUMA.

\subsubsection{Imagine}

The Imagine stream processor [57] developed in Stanford University is a programmable architecture for graphics and video signal processing. It is a parallel architecture with eight ALU clusters and each cluster is a VLIW processor core with six parallel arithmetic units. Each cluster executes an independent kernel program and performs arithmetic computing only on local data. Data locality is explicit in a kernel. There is no direct reference to global data from a cluster/kernel, instead of that, a cluster consumes input streams and produces computing results as output streams. Since the stream data are transferred in bulks, the memory 


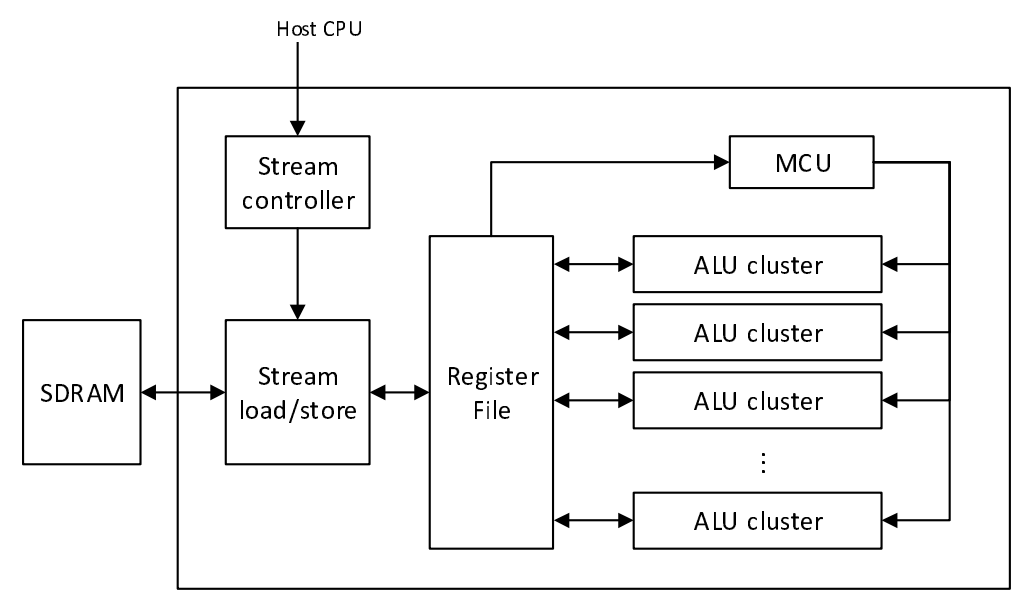

Figure 3.4: Imagine stream processor architecture

subsystem can be optimized for high bandwidth and low overhead. The stream controller handles bulk data read and write, rather than individual data access, the silicon cost of the memory subsystem is lower than conventional interconnects. The Imagine processor implements a three tired memory hierarchy, which contains a four-bank off-chip SDRAM, a $128 \mathrm{~KB}$ stream register file (SRF), and cluster local registers. The SRF is a shared register pool for all streams for the eight clusters. The data transfer of streams in the SRF is scheduled by the stream controller.

The Imagine processor is programmed using a stream programming model [58], which uses computing kernels and data streams to describe a signal processing application. The computing kernels run on the VLIW cluster of Imagine. And the memory subsystem moves streams between clusters and external memory. The software development for Imagine is divided into application level programming and kernel level programming. The application level software handles data streams, it is also known as stream level programming. The kernel level software operate on one element of a stream at one time, the same operation is applied to all elements of the stream. Kernels can only operate on input and output streams, it can not access the shared memory directly. 


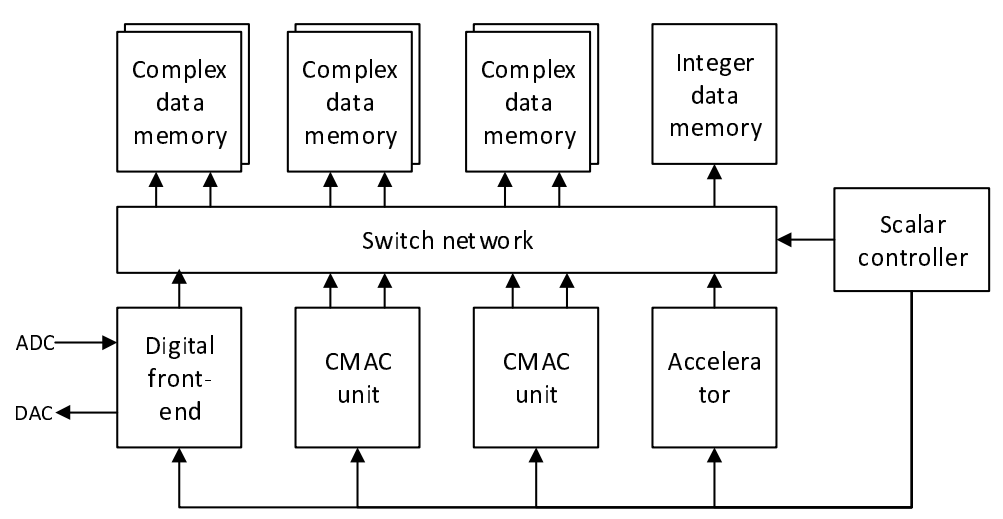

Figure 3.5: BBP baseband processor architecture

\subsubsection{BBP}

The BBP [5] is a programmable baseband processor for multi-standard baseband signal processing. The architecture is characterized as Single Instruction Multiple Task, as shown in Figure 3.5. The hardware components of BBP include the scalar controller, the SIMD vector execution units, accelerators, memory banks, and interconnection network. It uses a single instruction flow which contains two types of instructions, the scalar instruction executed by the controller core and the vector instruction executed by the SIMD execution units. The vector instructions usually take multiple cycles to complete. The scalar core can issue multiple vector instructions to different SIMD units to compute in parallel. The vector instructions and the SIMD computing units are designed specifically for baseband signal processing, for example complex MAC unit for FFT computation and complex ALU to perform pilot tone extraction. To provide parallel data access for the concurrent computation tasks, the BBP implements multiple memory banks, and uses a transitioned memory architecture to switch the connection between physical memories and processing units. The memory switch is controlled by software.

The BBP uses a single program flow in which only one instruction is issued every clock cycle. Task level parallel execution is initiated by inserting vector instructions in the program flow. The memory subsys- 
tem, including the address generation units for the memory banks and the memory switch, is also controlled by software.

\subsection{Summary}

In this chapter we introduced three types of parallel architectures: Superscalar and VLIW architectures for instruction level parallelism, a SIMD architecture for data level parallelism, and a multicore processor for task level parallelism. The computing performance of these architectures are mostly limited by memory accesses such as stream data transfers, parallel data access, and external memory bandwidth. Other limiting factors such as memory coherency and control overhead are discussed. Two parallel architecture examples are introduced: the Imagine stream processor for graphics and multimedia computing and the BBP processor for baseband signal processing. 


\section{Chapter 4 \\ Parallel Memory and Conflict Free Data Access}

This chapter introduces the fundamentals of the parallel memory architecture and conflict free access, and its implementation in the ePUMA SIMD processor.

\subsection{Introduction}

Most parallel processor architectures, such as VLIW and SIMD architectures, increase the computing capability by integrating multiple arithmetic computing units (ALU and MAC) in the datapath. These computing units can process multiple data in parallel. However, the parallel datapath can only be fully utilized if the parallel data can be accessed at a corresponding speed. Otherwise the data access overhead will limit the overall performance. Two major factors that can affect the computing performance of a parallel datapath are identified as:

- External memory access latency

- Parallel data access conflicts

The solution is to use multi-level memory hierarchy and parallel onchip memories as intermediate data buffers to minimize data access overhead and achieve high parallel computing efficiency. 
Multi-level memory hierarchy is a common approach in memory subsystem design to hide data access latency to the external main memory. For cost considerations, DDR off-chip memory is still the mainstream solution for large data storage in embedded systems. The DDR memory implementation saves silicon cost by minimizing the number of gates per bit, but it requires a complex data read and write protocol [55][59]. Data access to external memory usually goes through local cache or memory, on-chip network and external memory controller, the network arbitration and memory request queue further increases the access latency. Using multiple external memories for single DSP chip is not very common except for high performance systems like radar systems, since the interface to the external memory requires a large number of chip pins, which is expensive. Typical embedded DSP chips use one or two external DRAM memory devices.

In a multi-level memory hierarchy, data are preloaded to on-chip buffers before computing, and the computation result is written back from the on-chip buffer to the main memory. The per-bit silicon cost of on-chip memory (SRAM) is higher than external memory so the size is usually small. Typical implementations of the on-chip memories include cache and SPM. The cache system relies on hardware to exploit both time and spatial locality of data and keep frequently accessed data on-chip for fast access from the processor core. Cache based memory subsystems are suitable for instructions and non-predictable data accesses in general applications. In streaming signal processing, where a large part of the data accesses are regular and predictable, software controlled SPM architecture can provide more efficient data access and predictable performance.

This chapter focuses on a SPM based parallel memory architecture using multi-bank on-chip memory as processor local storage to provide fast vector data access for SIMD computing. The data movements between the parallel on-chip memory and the external memory are controlled by the DMA engine. The software also controls the data allocation in the parallel memory for conflict free access in SIMD computing. The design 
challenge for conflict free parallel memory access comes from the variety of data access patterns, which is application and algorithm dependent. Theoretical studies of the possibilities of conflict free parallel data accesses to a set of constrained access patterns was published in [16]. In this thesis, we focus on the concrete and practical conclusions.

This chapter will introduce the fundamental concepts and the model of parallel memory architecture (Section 4.2) and list a number of practical conclusions for common parallel data access patterns in modern DSP kernels (4.3). In contrast to book [16], which designs and optimizes specific address generation circuits for different memory access formats, this thesis instead proposes a generalized lookup-table based AGU architecture for recursive addressing (Section 4.4).

\subsection{Multi-bank Memory Architecture}

\subsubsection{Fundamental Concepts}

This section introduces the fundamental concepts in parallel memory access. The concepts are used in the following discussions of parallel memory model and conflict free access theories. For better illustration, we use a 2D array to demonstrate the data stored in the memory. The parallel access to the $2 \mathrm{D}$ array means to read out or write into multiple data elements at different addresses in the memory at the same time.

\section{Access pattern}

The access pattern contains the relative position information between elements in one parallel access, as shown by the groups of grey boxes in Figure 4.1. Examples of access patterns include straight line, rectangle, and cross. The access pattern is usually expressed as an ordered set of offset values from the first data element. For example, the access pattern of a straight line is expressed as $[0,1,2,3,4,5]$. And the cross pattern is $[0,-24,24,-1,1]$, where 24 is the picture width. 


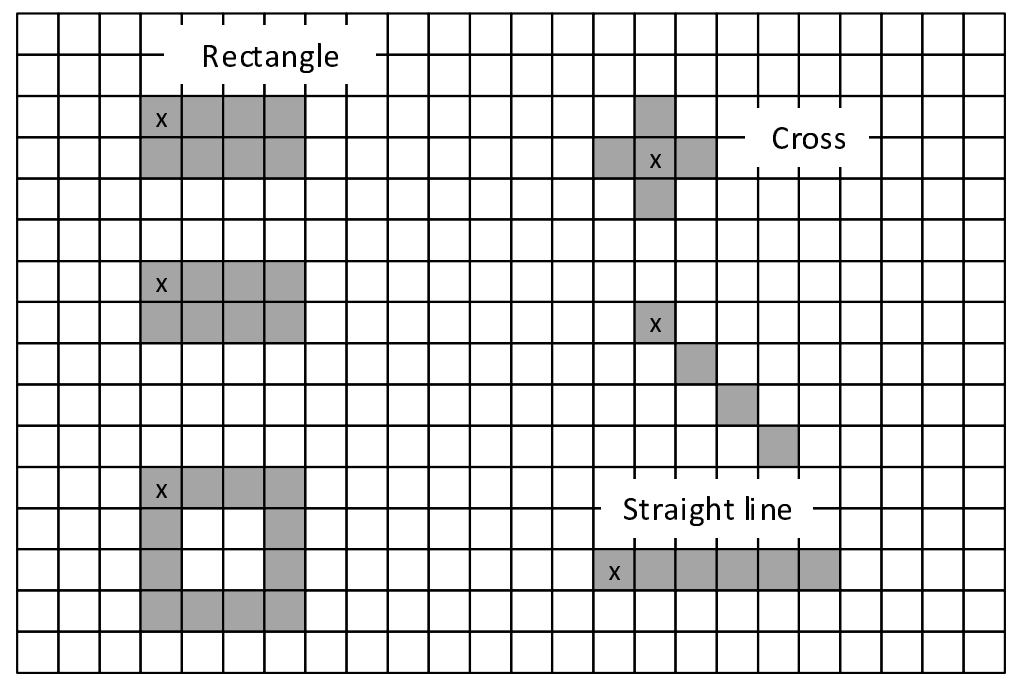

$\mathrm{x}$ Emphasize point

Figure 4.1: Examples of parallel memory access

\section{Emphasize point}

The first data element in a parallel access is called the emphasize point, as masked by ' $x$ ' in each pattern in Figure 4.1. The addresses of all the other data elements are calculated based on the emphasize point and the access pattern with the relative position information.

\section{Placement set}

The placement set is the collection of the emphasize points of all parallel accesses required by the computing unit. It determines the required parallel data accesses to the memory region.

\section{Module assignment function}

The module assignment function defines the memory module for each data in the parallel memory. The parallel memory architecture uses mul- 
tiple memory modules (banks) to provide parallel data access. The data allocation in the multi-bank memory is the key to avoid bank conflict in parallel access.

\section{Address function}

The address function determines the local address of each data in its assigned memory module. This function is less important than the module assignment function since for a given module assignment function, there exists many different address functions. The benefit that one can get from address function design is the reduced memory usage for a block of data, such that the permuted data do not expand to a large memory space.

\subsubsection{Parallel Memory Architecture}

The basic requirements of parallel memory architecture include:

- Parallel data interface: the processing units (processor core, accelerator and DMA controller) can read and write data at high data rate, typically one vector per cycle.

- Recursive address generation: the AGU can generate addresses for all memory modules repeatedly for stream data access.

- Flexible data access: the read and write operation to the parallel memory can access any address in each memory bank, and in any order between memory banks.

The reason for the first requirement is obvious. Parallel data access is important to achieve high ALU utilization of parallel data path. The second requirement is beneficial for hardware accelerators and DMA controllers, where the AGU is pre-configured by software and the address generation is triggered by the read or write enable signal. It can also be useful when the processor instruction set does not include enough coding space to support complex addressing. Then the data access is configured before computing a kernel, and the address generator executes in 


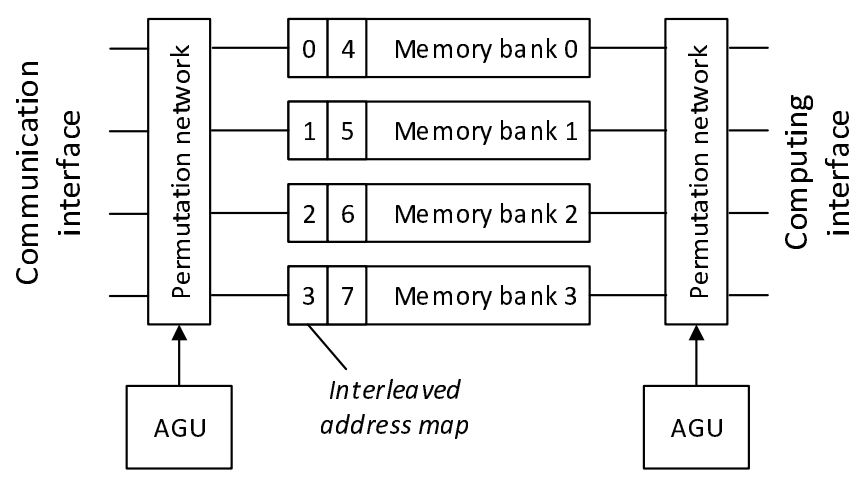

Figure 4.2: Parallel memory architecture model

parallel with arithmetic computation. The third requirement is for flexible parallel data access of different access patterns. For example horizontal line, matrix diagonal, and 2D block, as illustrated in Figure 4.1. This is achieved by providing each memory with an individual input address and using a shuffle network to permute the vector data.

A theoretical model of parallel memory architecture is shown in Figure 4.2. It consists of a multi-bank memory, parallel address generator, address decoder and permutation network. An interleaved address assignment is used in this parallel memory to ease the decoding of module number by using the LSB bits of the address. Each memory cell has a unique address in the parallel memory. The AGU will generate a vector of addresses for the parallel access. For write operations, each address ends up in one memory bank, no two addresses generated by the AGU at one time can conflict in one memory module. For read operations, it is allowed to have the same addresses in one address vector. The address decoder takes the vector address as input, and decodes the local address of each memory bank. The permutation network shuffles data from the memory bank order to the access order as specified by the address vector. 


\subsection{Conflict Free Parallel Memory Access}

For single port memory, within one clock cycle it is only possible to access one address in each memory module. This limitation causes the memory conflict problem in parallel data access when two or more accesses are on the same memory module. Theoretical study [16] has shown the relationship between the access pattern requirements, the placement sets, and the minimum number of memory modules that can support the conflict free access. However, in practical implementations only $N=2^{m}$ memory modules are relevant. It is easier to support different data types by one parallel memory, e.g. 16 bit fixed point and 32 bit floating point. The number of memory banks should be as small as possible to save silicon cost. Usually the number of memory banks is determined by the parallelism of the data path. For example an 8-way SIMD core uses a 8-bank parallel memory to store local data.

The focus of this thesis will be on the problem of using restricted number of memory modules for conflict free parallel access. The following will list a number of useful conclusions in conflict free parallel memory design, with a restricted number of memory banks of 8 as in ePUMA. The access patterns will be focusing on straight lines and rectangles, which are common access patterns in computing kernels like filtering, matrix operation, and transform.

Conclusion 1: For parallel memory with 8 memory banks, it is possible to realize conflict free access to two rectangle patterns of $2 \times 4$ and $4 \times 2$ blocks simultaneously without any placement set restrictions.

Conclusion 2: For parallel memory with 8 memory banks, it is possible to realize conflict free access to horizontal and vertical straight lines consisting of 8 data points without any placement set restrictions.

Conclusion 3: For parallel memory with 8 memory banks, there exists no module assignment function which is conflict free for horizontal and vertical straight lines, and $2 \times 4$ and $4 \times 2$ rectangles at the same time, if the placement set of the rectangles are not restricted. For re- 
stricted placement set of rectangles, it is possible to find a module assignment function that can support all access patterns simutaneously.

In practice, we can moderate the parallel access restrictions by allowing the parallel accesses to be completed in multiple cycles. We can still get significant improvement on overall system performance.

The design challenge for software developers is to find the module assignment of each data in the parallel memory for the maximal level of parallel access. For simple access patterns, software developers can implement linear and periodic assignment functions without much difficulty. But for complex algorithms with different access patterns and placement set restrictions, tools developed based on integer liner optimization could help to find the optimal module assignment funtion. Such a tool [60] is implemented in ePUMA tool-chain.

\subsection{Lookup-Table Based Address Generation}

The module assignment functions in practice are mostly introduced by a recursive definition, which means that only periodic functions are considered. This forms the basis of our AGU architecture design using recursive address generation. Recursive addressing is suitable for DSP computing, where the computation intensive kernels are usually the innermost loop which processes an input data stream in a regular order. The address generation can be pre-configured before entering the loop, so that the address computation and the memory access will have no extra cycle cost during kernel computing. However, the addressing flexibility of the parallel memory architecture requires a more complex AGU architecture design, which needs to generate information about both the emphasizing points and the vector access patterns. This leads to the lookuptable based AGU architecture, as shown in Figure 4.3

The AGU model has two address branches, the scalar branch and the vector branch. The output address to the address decoder is the addi- 


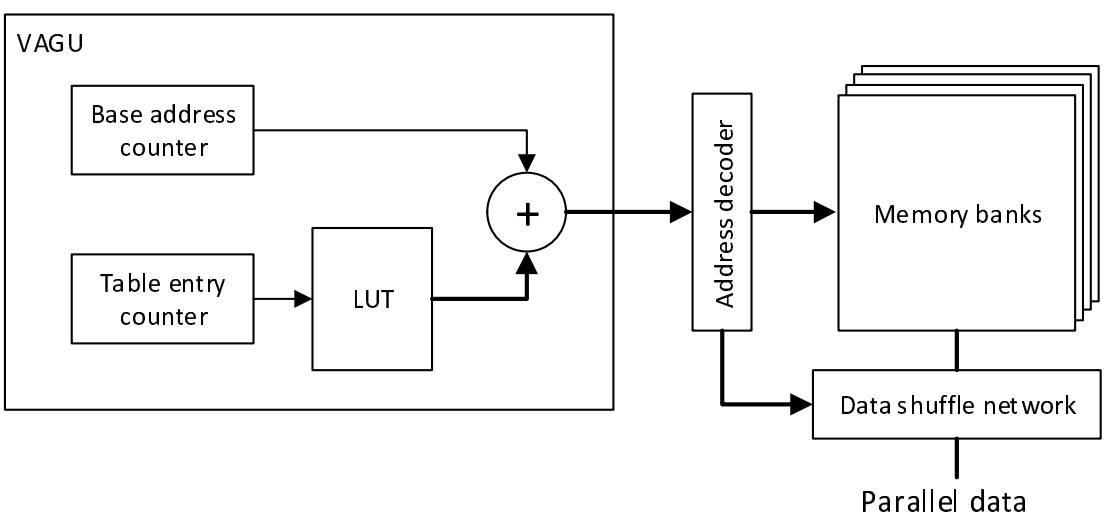

Figure 4.3: Lookup-table based AGU architecture

tion of scalar branch address and vector branch addresses. The scalar branch calculates the emphasizing point, based on the conventional addressing of 1D stride, modular, bit reverse, and 2D stride. The vector branch is based on a lookup-table to store the access patterns. The entry to the lookup-table is controlled by a address counter similar to the scalar address generator, which supports 1D stride, modular, and 2D stride addressing.

This AGU architecture in ePUMA is used to generate address sequences when transferring stream data. The SIMD engine integrates a similar AGU in its pipeline and exposes more control to the software instructions. The lookup-table of the AGU uses the program memory of the SIMD engine, which contains read-only data.

\subsection{Summary}

In this section, we have introduced fundamental theories of conflict free access and the parallel memory architecture model. The theoretical research conclusions show the possibilities of conflict free parallel access for different access patterns. A generalized vector AGU architecture for the parallel memory is proposed. It performs iterative address gener- 
ation based on a lookup table. For predictable computing algorithms, the address patterns can be preconfigured in the LUT memory. The parallel memory architecture solves data permutation in on-chip memory instead of the vector registers by using permutation instructions to control the AGU. It saves both the execution time on data preparation for computing on parallel datapath and the hardware cost on large register file used for data shuffling. 


\section{Part II}

\section{System Architecture}





\section{Chapter 5 \\ ePUMA: A Multicore DSP Architecture for Streaming Signal Processing}

In this chapter, the contributions of ePUMA parallel architecture design and the stream-oriented parallel programming model are described in detail.

\subsection{System Overview}

ePUMA is a multi-core parallel DSP processor designed for streaming signal processing applications which are dominated by predictable computing kernels. Such applications include multimedia, communication baseband, and graphics. ePUMA uses a chip multiprocessor (CMP) architecture that combines one master processor and multiple DSP coprocessors. The DSP coprocessors use a SIMD architecture to gain high computing power from data parallel execution. ePUMA CMP architecture explores both task level parallelism and data level parallelism. The master-SIMD combination separates the main program flow from the kernel computing. The master core controls the top program flow, and the computing intensive kernels are executed on multiple SIMD coproces- 
sors in parallel. Each SIMD processor further separates the data access from parallel arithmetic computing. By separating the communication and computing operations, data access and kernel computing are orthogonal to each other and can be executed in parallel, such that the overhead of data access can be minimized.

\subsection{Architecture Design Decisions}

The ePUMA architecture is designed after an investigation of various processor micro-architectures, parallel programming models, on-chip interconnection architectures, and memory subsystem architectures. The design decisions are summarized as follows.

\section{Master-slave multiprocessing}

Conventional single core DSP processors only have one instruction stream which is executed sequentially. The computing kernels which are optimized for fast execution have to be executed in a sequential way. Multicore DSP systems usually have each DSP core as an independent subsystem. Each subsystem can generate data access requests through its bus master port. As the number of DSP cores increases, the data traffic on the bus and the data access to the external memory can be very random in such systems. ePUMA uses a master-slave architecture to manage computing kernels to run on multiple slave processors in parallel. There is only one master that runs the main program of an application. The master processor manages data communications with the external memory. Slave coprocessors only process streams assigned by the master core. The streams can contain not only the computing data, but also the computing commands using predefined data structure.

\section{SIMD engine for kernel computing}

SIMD architecture has been widely used in embedded signal processing for its small code size and efficient parallel datapath. The data parallel ar- 
chitecture is suitable for image processing, video codec, communications, and graphics applications. ePUMA uses a SIMD architecture to build the slave processors. Each slave coprocessor contains a SIMD datapath which can perform $16 \mathrm{MAC}$ operations in one clock cycle. It means the equivalent computing capability of running one 16-MAC, two 8-MACs, or four 4-MACs in one clock cycle. The vector data width of the SIMD engine is 128 bit. It can be used for 16x 8-bit, 8x 16-bit, or 4x 32-bit parallel computing. ePUMA SIMD arithmetic instructions also use memory operands directly to save load/store overhead.

\section{Kernel based and stream oriented parallel computing}

ePUMA uses a kernel based parallel programming model. From the programmer's point of view, the kernel based parallel programming model is easy to use. It is similar to the conventional DSP programs that use DSP library functions to accelerate critical computing tasks, but in ePUMA the input and output of the kernel functions become data streams managed by the master core. The execution of a kernel function is triggered by data streams. In this programming model, each kernel operates on its own input data streams prepared by the master controller. The master controller collects output stream from the kernel after computing. The stream data are stored in the shared memory region and transferred to the coprocessor's local memory by the DMA engine controlled by the master core.

\section{Distributed memory architecture}

ePUMA chooses to use a distributed memory architecture. Each SIMD processor has a private local memory. One reason to use distributed memory is to implement the parallel memory architecture which can reorder the input data for conflict free access during kernel computing. Another benefit of using distributed memory is that it avoids the memory coherency problem of multi-core architectures. In ePUMA, only the master core can access the main memory, the kernels running on SIMD 
coprocessors only process data in the local memory. The master core controls the update of the global memory data from SIMD local memories. It manages the data streams to be transferred in the correct order to maintain data coherency.

\section{Parallel memory and LUT based AGU}

The purpose of implementing the parallel memory architecture is to improve SIMD computing efficiency by enabling conflict free parallel data access. In ePUMA, the parallel memory is used in the LVM (Local Vector Memory), which consists of 8 memory modules to support 8 data accesses in parallel. Each memory bank of the LVM is 16-bit wide. The AGU for memory address generation is based on a lookup table. The same AGU architecture is used by both the DMA controller and the SIMD core. Data permutations at both DMA and SIMD side enables the flexibility in parallel data access .

\section{Triple physical memory buffers}

Instead of using multi-port memory to support concurrent data access for the DMA engine and the SIMD core to the local data buffer, ePUMA uses three single port LVM modules in each SIMD processor and switch the memory buffers for communication and computing during kernel execution, as shown in Figure 5.1. This design decision is made to reduce the silicon cost and power consumption on expensive on-chip memory: normally a two-port memory consumes about double silicon area of a single port memory of the same size. A design example of a delay buffer in a baseband processor shows that using two single-port SRAMs of size 2048 bytes to replace a two-port memory of the same size can save $30 \%$ power and $50 \%$ area in the 0.18 um CMOS technology [61]. The three physical memory buffers are connected to the DMA controller and the SIMD core through a LVM switch, which is controlled by the SIMD processor. At run time, one of the three LVMs can be connected to DMA to transfer data. The other two LVM modules are connected to the SIMD 


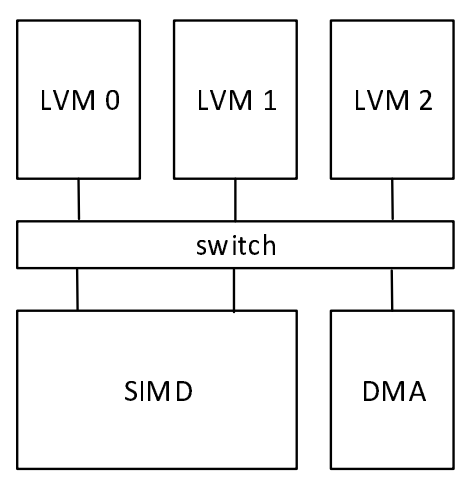

Figure 5.1: Tripple memory buffers

datapath for kernel computing. All of the three LVMs can be connected to the data-path to support high throughput computing such as matrix computing, in this case the DMA communication is disabled during SIMD computing.

\section{Multi-layer network}

The interconnection network of ePUMA needs to support the following types of data communications:

- Off-chip stream data transfer to external main memory which is shared between ePUMA and other hardware modules in the system.

- On-chip stream data transfer between SIMD processors.

- Short data transfer for passing parameters and messages between processor cores.

The stream data transfer moves a block of data from a source memory to a destination memory. The off-chip communication bandwidth can be affected by the system interconnection bus and the memory controller hardware IP. The communication might be interrupted by other transactions on the system bus. The second type of inter-SIMD communication 


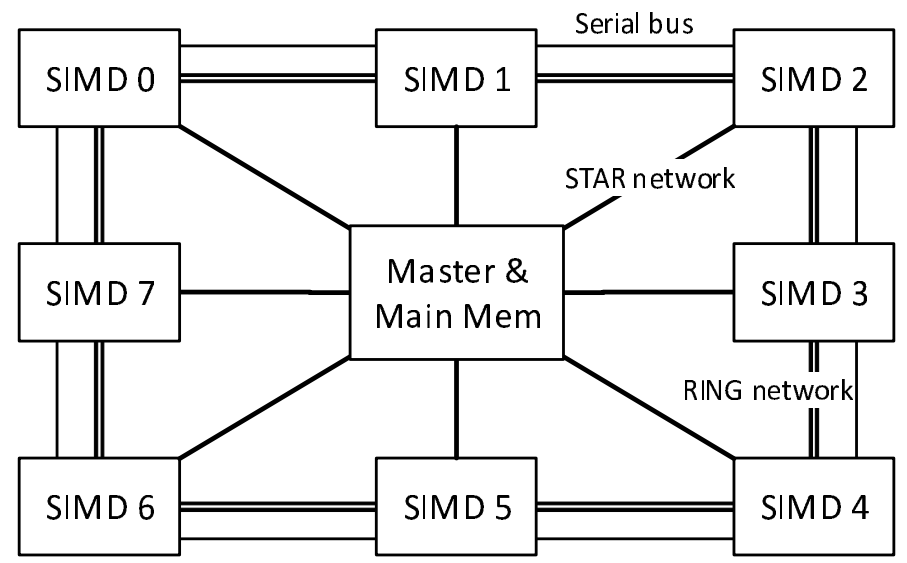

Figure 5.2: Multilayer network

is not affected by other hardware modules outside ePUMA. If a physical link was setup and dedicated to that communication task, it can exhaust the full bandwidth to transfer the data stream. The short data transfer is used to send a few data message such as control information and function parameters to a target processor. Low latency is more important for this type of communication.

ePUMA implements a multi-layer network which combines a Star network, a Ring network, and a serial bus, as illustrated in Figure 5.2. The Star and Ring stream networks together support master-slave and data-flow parallel computing models. The Star network is based on a multiport centralized DMA controller [52]. It connects the master controller with all SIMD processors. The Star network supports data movements between external memory and processor local memories. Data stream on the Star network include SIMD kernel program and vector data for SIMD computing. In the Star network, the master processor and the DMA controller locate in the star center. SIMD processors are connected to the Star leaf nodes.

The Ring network is based on circuit switching [62]. It connects all SIMD processors using a ring topology. One SIMD processor can send a data packet through the Ring network to one or multiple target SIMDs. 
The physical layer of the Ring network is configurable at design time to use different hardware resources to provide different concurrent communication bandwidth. The configuration parameters include the number of ring layers and the size of data buffers.

The serial bus is designed for mailbox communication. It is used for inter-processor short data communication and synchronization. It has a fixed latency to send data from the source to the destination mailbox registers. The physical link layer design is simplified. The sender uses a send-and-forget scheme, and the receiver mailbox registers are read-andclear. Complex handshake protocols should be implemented in software on top of the physical layer protocol.

\section{Non-address NoC with message passing}

The Star and Ring networks are dedicated for stream data transfer. The network doesn't transfer address information. The network arbitration is done at stream level. There is no limit on the length of a stream, which is determined by software when configuring the DMA task. The address calculation is done at the transmitter for source memory and at the receiver for destination memory. This non-address stream network is hardware efficient for stream data transfer. It is suitable for ePUMA's distributed memory architecture and the LUT based local address generation. The network does not need to transfer address information using an address bus, which usually takes a significant amount of network bandwidth and hardware resources.

\section{Data broadcasting}

Data broadcasting is a typical communication model in parallel signal processing. It requires a common data to be sent to multiple destinations for computing. However, most existing interconnection architectures are designed to implement point-to-point communications only. Collective communications such as broadcasting and reduction are supported by higher level APIs build on top of the basic point-to-point communication 
Processing

functions. Except for better programmability, the software implementation has no advantage in performance. Implementing broadcasting using multiple point-to-point transactions is a big waste of the network bandwidth.

ePUMA Star and Ring stream networks supports broadcast communication in link layer. Data broadcasting is completed by a single transfer on the network. Data broadcasting on the Star network is implemented by the multi-port DMA controller. It can reduce the external memory bandwidth, which is an expensive resource for DRAM based signal processors. The broadcast communication from one SIMD processor to multiple other SIMDs is supported by the Ring network. For the Star and Ring communications, the address information is not transmitted along the network, we only need to specify the destination processors for a data stream which needs to be broadcasted.

\subsection{Multicore Computing Models on ePUMA}

The dilemma in multicore parallel computing is how to map applications to multiple processing cores. The multiprocessor architecture brings new challenges for software developers to parallelize tasks for concurrent execution, and more importantly, to fully utilize the hardware capabilities through explicit software control. ePUMA on-chip network combines a Star network and a Ring network, which by nature support the dominant three multicore computing models [45]: master-slave, data flow, and a combination of these two models. The master-slave model has one master core which controls the work assignments on all cores. In the data flow model, work is passed through a pipeline of processing stages. The combination model uses one master controller, and also allows data flows between slave cores during execution.

The master-slave model is suitable for executing independent tasks over multiple processing cores. The task of the master core is to balance the computing load across the slave cores to achieve optimal parallelism. The challenge for this model is dynamic scheduling of kernels because 
the task configuration and processing load can change at run time. One example application is the multiuser data extraction and processing in the uplink of LTE physical layer. The system configuration can change on different channel conditions, and the user bandwidth resources also change under system control. All of these changes will result in different computing load of signal processing kernels such as the size of FFT and the MIMO detection algorithm computing complexity. Multiple light weight kernels can be grouped and mapped to a single slave core. Master controller trigger a kernel execution by sending input stream to it. The slave core stops after finishing a kernel function.

The data flow model follows the signal processing pipeline of the application algorithm, where one or multiple processing stages are mapped to one core, and the execution on each core depends on the output from the previous stage. The processed data are transferred between processing cores through the on-chip network by DMA transfers. The initial core reads in the source data from global memory or sensor. Its execution is triggered by events such as timer or input sensor. In ePUMA, the initiator can be the master core or one SIMD core with an extended sensor interface which streams in data directly into the LVM memory. The LVM switch provides such capability to extend the SIMD core with sensor interface or ASIC accelerators. Most signal processing applications fit the data flow model because the algorithm is based on a pipeline of computing stages, and the computing stages are dependent on each other. The challenge of parallel computing using this model is on task partitioning. The programmer needs to consider both computing load on each core and the inter-processor communication load.

The third model combines master-slave and data flow models. It is commonly used in ePUMA in practice. The master manages several SIMD processors for one application. It runs the main program. The kernel programs for SIMD processors are loaded by the master controller. The kernel computing is triggered by input data stream, which can be sent from the master core through the Star network or from another SIMD processor through the Ring network. One SIMD kernel can issue 
Processing

data transfers to another SIMD core independently from the master's control. The master core can run multiple application tasks at the same time. It allocates a group of SIMD cores for one application, and several other SIMD cores for another application. The master core switches between multiple applications at the top program level. Since the slave cores are blocked by data streams, it will wait for the master to switch back to its application task during multitasking.

\subsection{Inter-Processor Data Communication}

\subsubsection{Data Memory Placement}

The data placement includes the placement of software program data and computing data in the memory hierarchy. Each application program is a single image which contains master program and SIMD kernel subroutines. The software image is loaded to the external memory from non-volatile storage during initialization. Ideally, all program data is allocated to on-chip SRAM before execution. The master program memory (MPM) is loaded by the host system during global initialization. And the SIMD program memory (SPM and VPM) is loaded by the master core during local initialization. However, the on-chip memories usually have space limitations. To support applications with large code size, the master core can be extended with an instruction cache to access the external memory. A typical large application will use the MPM for runtime critical functions, and allocates non-critical sections to the external memory.

SIMD processors have high code efficiency, the program size of SIMD kernels are usually very small. The on-chip SIMD program memory only keeps a small number of kernels during execution. SIMD kernel program can be swapped at runtime by the master core. Therefore it can achieve a very efficient use of on-chip memory. The data transfer overhead of swapping kernels can be mostly hidden in predictable applications. The kernel program code is loaded to the SIMD core to execute locally. There is no shared kernel code or kernel data on the external memory at run 


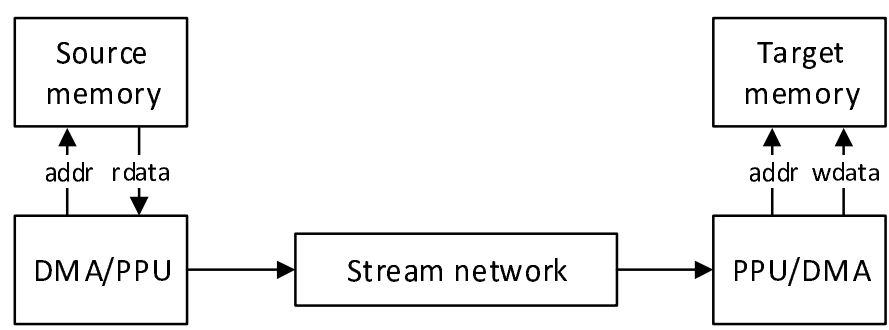

Figure 5.3: Stream data movement from source memory to target memory

time, which simplifies the software complexity of code sharing and memory coherency.

\subsubsection{Stream Data Movement}

In the ePUMA multicore system, the primary data transfer engines for stream communications are the central DMA controller and the SIMD PPU engines. The DMA controller is controlled by the master core, and each PPU engine is controlled by its local SIMD core. As shown in Figure 5.3, the data movements from a source memory to a target memory are performed by the cooperation between two communication engines, for example one DMA controller with one Packet Processing Unit (PPU) for data communication between the external memory and the SIMD local memory, or two PPUs for stream data transfer from one SIMD local memory to another SIMD local memory. One engine is responsible for getting data from the source memory and sending the data stream to the communication network, while the other engine receives the data stream from the network and writes the data to the target memory. The source and destination engines need to synchronize with each other to perform a data transfer. In the broadcasting mode, multiple SIMDs receive data from the same source, the source communication engine (DMA or PPU) sends data to all synchronized target PPUs by one transaction.

The central DMA controller is used when data are transferred to or from the external memory. The data streams include kernel program data and computing data. Kernel program transfer from the external mem- 
ory to the local SIMD program memory requires the SIMD processor to be in the idle state. When the SIMD core is started, the program memory is switched from the communication network to the processing core.

In each DMA or PPU engine, there is a hardware queue to store configuration parameters of communication tasks. Multiple tasks can be linked together and will be completed by one DMA execution. The engine starts execution from an arbitrary task specified by the processing core, and continues until all the linked tasks are finished. The central DMA engine contains a hardware queue of 16 tasks, and each PPU engine has a hardware queue of 4 tasks.

\subsubsection{Notification and Synchronization}

Multiprocessor platforms require that the communication architecture supports notification and synchronization between cores. The ePUMA master-slave and distributed memory architecture brings about a much simplified protocol for notification and synchronization across multiple processing cores. The master core is responsible for all the system initialization and the slave cores must wait for commands or input streams from the master to start execution. When the kernel computing is finished, the SIMD core can notify the master core. Notification can be implemented using interrupts or register polling. The polling method is preferred for predictable computing since the master core only checks the state register when it intends to do. It reduces the context switching load of the master core when interrupt is used.

ePUMA's communication architecture is optimized to reduce the control overhead by data streams in predictable applications. Its advantage is when data movements are scheduled before execution, the inter-core notification for data transfer can be eliminated. The processor core sends a communication command to the data transfer engine and continue executing other parts of the software. It does not need to interrupt and notify the other core at the other side of the communication link by a long latency handshaking protocol. The data transfer is synchronized in 
hardware by the DMA and PPU engines.

For other non-predictable data transfers, ePUMA supports different inter-core notification methods to exchange information between cores. These notification methods can be used to send short data message directly, or exchange parameters for configuring the stream data transfer engines to move a large volume of data through the Star and Ring network. The different software protocols for notifications are based on the hardware implementation using different types of control registers, including:

Write-to-interrupt register When an event value is written to this register by a transmitter, it generates an interrupt event to the interrupt controller to notify the receiver core. The value kept in the register indicates the type or source of interrupt.

Read-and-clear register When the register is read by the receiver core, it automatically clears the data. The data written to this register usually contains a valid bit, so that the core can detect valid message.

Trigger register This type of registers do not keep the write data, it sends a one cycle event to the hardware block as a control signal. It is typically used for sending start signal or edge triggered interrupt.

Normal registers The last type is the normal register without any sideeffect operation.

Each SIMD core has two sets of mailbox registers, one for the master core, and another for SIMD to SIMD notification. The master core accesses SIMD mailbox registers through the memory mapped I/O. The SIMD core sends message to other SIMD's mailbox through the serial bus.

\subsection{Host Interface to DSP Subsystem}

ePUMA as a DSP subsystem is controlled by a host controller. It has two operation modes: standalone application and standard API interface. 
Processing

\subsubsection{Standalone Application}

The standalone application mode allows ePUMA subsystem to run applications with a minimum interaction with the host CPU. The DSP subsystem is initialized by the host CPU to run an application, for example a video decoder. The initialization include preparing the software image, reset ePUMA, load boot code into the master's program memory, and start ePUMA. The host CPU controls the ePUMA subsystem by using ePUMA's host registers. The master core's program memory is also mapped to the system memory space. The host CPU can initialize the master program memory directly. After initialization, ePUMA runs all the computing tasks in the decoding algorithm including the timing control using a local timer. If necessary, some system peripherals such as a video display module can be controlled by ePUMA's master core directly.

\subsubsection{Standard API Interface}

The standard API interface mode contains implementations of various software API functions which are called from the host CPU, for example OpenGL ES [63] for graphics, OpenMAX [64] for multimedia accelerations, and OpenCL [7] for general purpose parallel computing. ePUMA DSP subsystem is initialized by the host CPU for a specific API mode. The execution of API function is triggered by the host CPU which interrupts ePUMA's master core. The host CPU writes task descriptors in the shared memory to pass API function parameters to ePUMA DSP subsystem. The data structure of a task descriptor depends on the software implementation of the library function. The host CPU has the flexibility in software to manage a task queue of API function calls and choose different notification frequencies. The notification to ePUMA subsystem can be immediate interrupt or delayed interrupt. The immediate interrupt sends interrupt as soon as a new task descriptor is put into the queue by the host. The master core of ePUMA receives interrupt and switches to the handler function to process the descriptor immediately. 
In the delayed interrupt notification, the host CPU decides the frequency of interrupts. The software sends interrupt only when a number of task descriptors have been put into the queue. The delayed interrupt notification reduces the interrupt frequency and thus reduces the context switching load on ePUMA master core.

\subsubsection{Multitask Execution}

A more complex use case of ePUMA DSP subsystem is to execute multiple applications at the same time, including standalone applications and API function calls. The master core of ePUMA is a typical RISC controller which can run simple multitasking OS and switch between applications. Since most of the computing load is allocated to the SIMD cores, the master core has more availability for OS operations. In the multitasking mode, different SIMD cores are allocated for different applications. The SIMD core at runtime is not shared between tasks. Task switching can be executed independently from the SIMD execution. The current multitasking framework supports only static tasks. Multiple applications and the OS is compiled into one software image. The host CPU can enable or disable a runtime task through the host control registers.

\subsection{Support for Debug}

The DSP system needs to support runtime monitoring of software execution and data traffic to debug application software. The tracing function is needed at different development phases, including simulator, prototyping and also field test. Trace logs can be generated by software instrumentation or by a hardware tracer. It is important to use as much software approaches as possible to generate trace logs in order to save hardware implementation cost. In the current version of ePUMA, we only use software instrumentations to generate trace logs. Extra execution overhead is introduced if the tracer function is enabled. 


\subsection{Summary}

This chapter gives a system overview of the ePUMA multicore DSP architecture. It describes the hardware architecture design and the software programming model of the multicore DSP system. The essential architecture design decisions are listed, including the master-slave multiprocessing, SIMD architecture for kernel computing, stream oriented parallel computing, distributed memory architecture, conflict free parallel memory, multi-layer network and stream communication. It also describes the interface to the host CPU for execution of standalone applications and standard API functions. The support for multitasking on ePUMA and data tracing for debugging is discussed at the end. 


\section{Chapter 6 \\ Memory Subsystem Design}

The contribution of ePUMA memory subsystem design and implementation is described in detail in this chapter.

\subsection{Introduction}

This chapter describes the implementation details of the ePUMA memory subsystem. The focus of this dissertation is on the on-chip memory architecture and the the multicore interconnection inside ePUMA. Other hardware components in the host system such as the system bus, shared memory, and memory controller are not within the scope of this thesis. The memory subsystem design includes the memory hierarchy, interconnection network, DMA controller, and processor local memory system.

\subsection{Memory Hierarchy}

ePUMA memory subsystem is based on SPM memories and software controlled data movements. The memory hierarchy consists of three levels, as shown in Figure 6.1.

- Level-1: processor local register file. This includes the master controller's register file and the SIMD controllers' vector registers. 


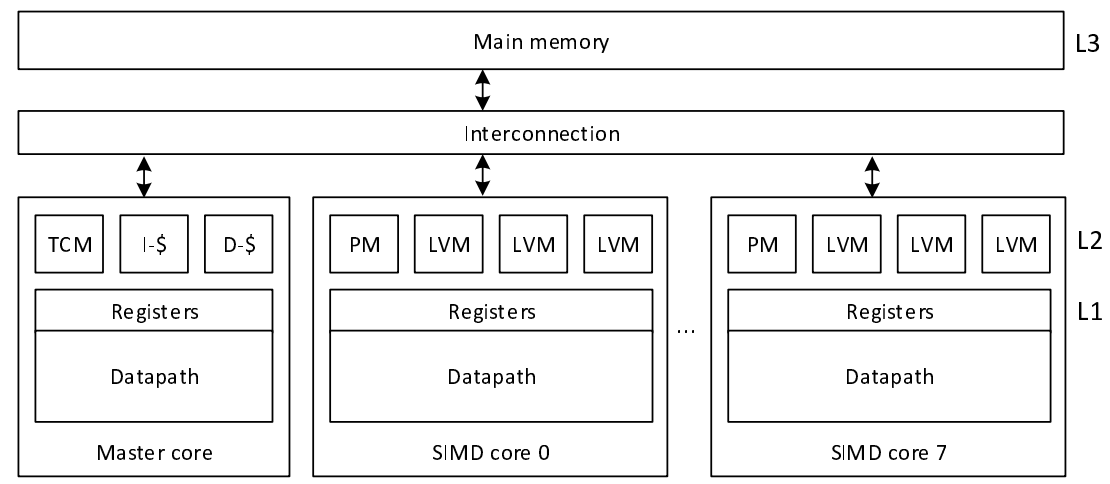

Figure 6.1: Memory hierarchy

- Level-2: processor local memory. For the master controller, this level contains the TCM memory and the instruction cache and data cache. For the SIMD core, it includes the program memory (PM) and three LVMs as data memory.

- Level-3: external shared memory. It is the highest level in the hierarchy with the longest access latency. The data access from the processor core to the external memory needs to go through the interconnection network and the memory controller.

The level-3 external memory has the largest size and is used to store program data and computing data. The processor local memory at the second level includes both on-chip data memory and program memory. The SIMD processors use eight-bank vector memory (LVM) as the local data buffer and a 32-bit wide memory for software code and data. Using a single memory for both code and data can simplify the compilation and kernel loading process. The lowest layer in the memory hierarchy is the register files in the master and SIMD processors. The SIMD instructions support both memory operand and register operand. During SIMD execution, the LVM memory is dedicated to the SIMD core for computing, which means the SIMD core can access level 1 and level 2 memory at the same speed at runtime. The level 1 registers have 1 cycle access latency, 
and the level 2 onchip memories have three cycles access latency.

Data in level-3 external memory need to be moved to level-2 for kernel computing. After computing, the results in the level-2 LVM are stored back to the external memory. Data movement between the external main memory and the level-2 SPM memories is done by DMA transaction controlled by the master core. In order to hide the communication latency, ePUMA implements ping-pong buffers for level 2 LVM memory. In this way DMA transaction can be overlapped with SIMD computing. The SIMD core uses load-store instructions to move data from level-2 SPM to level-3 register file. The load store instructions are mostly used for constant data, AGU parameters, or accumulation result. The overhead of data load and store in kernel computing can be eliminated by using memory operands directly. Furthermore, level 2 data can be moved from one SIMD core to another SIMD core through the onchip network. This is done by the PPU data transfer engines and the Ring network.

\subsection{Multi-Layer Interconnection Network}

ePUMA uses a three layer interconnection architecture which combines a Star network, a Ring network, and a Serial bus for data communications, as shown in Figure 6.2. The Star network and the Ring network are stream networks to transfer long data bursts. The Star network moves data between level-3 external memory and level-2 processor local memories. The Ring network moves data between level-2 onchip memories. The Serial bus is designed for short and fixed latency mailbox communication between SIMD cores. It supports data communication at register level (level-1). The Serial bus transfers data on a single bit data bus. All the communication channels are uni-directional, which is backend friendly. The hardware implementation can insert necessary register stages in the physical link to improve timing closure.

The purpose of the separation of network layers is to design dedicated networks for different types of communications, including streaming data transfer and short message passing. The combined Star and Ring 


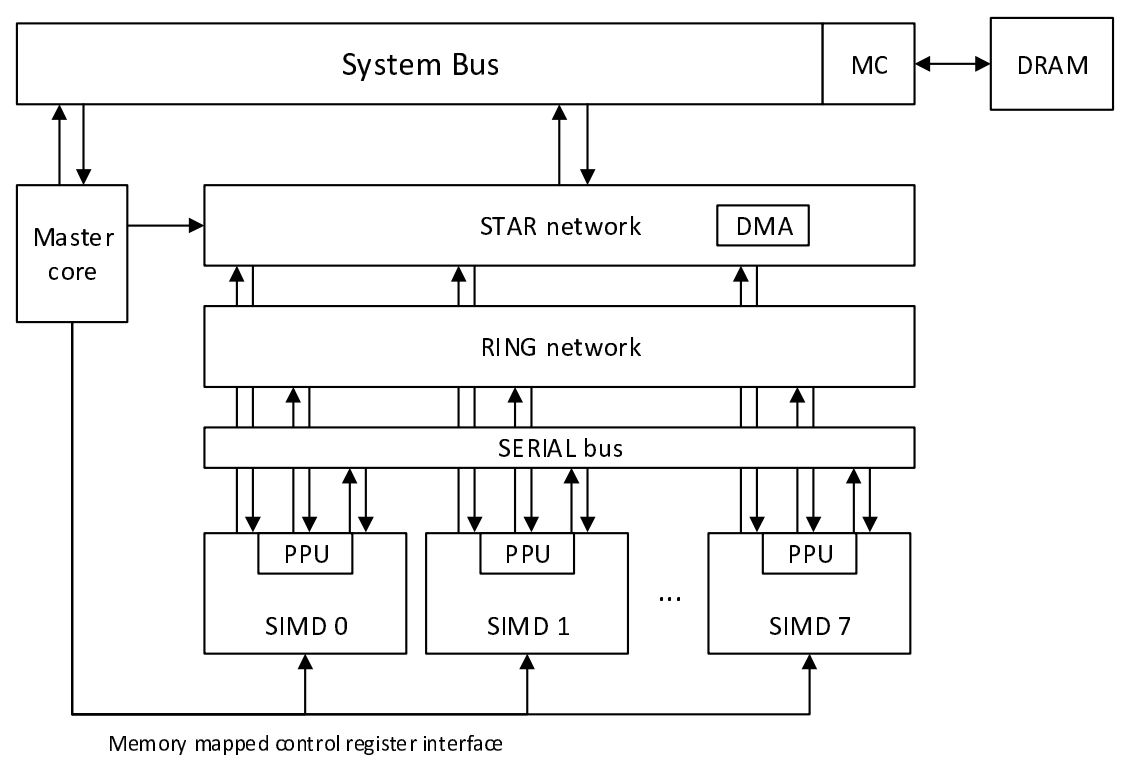

Figure 6.2: Multilayer network

network is to separate the external data traffic and on-chip data traffic, and support two multi-core computing models, the master-slave model and the data flow model. These two parallel computing models require different communications features. In the master-slave model, the communication is between the external memory and processor local memories. In the data flow model, concurrent on-chip data communications between processors are needed. We design the Star network with a multiport DMA controller for the master-slave model and the Ring network for the data flow model. Both of the two networks are optimized for the streaming data communication. The data streams and supporting networks are listed in Table 6.1.

\subsubsection{Stream Data Transfer}

The stream data transfer on the Star and Ring network uses a communication model called Tx+Rx. In this model, the data movement is ac- 


\begin{tabular}{|c|c|l|c|}
\hline Src. & Dst. & Data & Network \\
\hline MM & PM & kernel program & Star \\
MM & LVM & computing input data & Star \\
LVM & MM & computing result data & Star \\
LVM (SIMDa) & LVM (SIMDb) & inter-SIMD stream data & Ring \\
Reg. (SIMDa) & Reg. (SIMDb) & data message & Serial bus \\
\hline
\end{tabular}

Table 6.1: Data movements and corresponding networks

complished by a cooperation between the transmitter processor and the receiver processor. The transmitter processor reads data from the source memory and sends it to the network, the receiver processor gets data from the network and writes it to the destination memory. One can think of the network as a FIFO buffer, the transmitter puts data into the buffer, and the receiver pops the data from the FIFO.Using this communication model, the inter-processor synchronization for data communication is simplified. The FIFO network enables the transmitter and receiver to work independently and synchronize the communication by hardware. This is more efficient than software handshaking, which requires multiple cycles and may involve extra delays in interrupt handling. Another benefit we can get from this communication model is the saving of implementation cost. Since both the Tx and the Rx processors calculate the memory address locally, the data being transferred on the network is pure data stream. No address bus is implemented in the network. The stream data transfer is handled by the DMA and PPU engines in ePUMA. The DMA engine receives commands from the master core, and the PPU engine is controlled by the SIMD coprocessor.

The Tx-Rx model enables flexible stream data allocation in processor local memories. We define the kernel program as a subroutine or function which is compiled and run on the SIMD processor. In a distributed memory system, the kernel program controls the data allocation in its local memory. The kernel programmer shuffles data in the local multibank memory for conflict free parallel access during computing [65]. The 
kernel program will receive an input data as a sequential data stream, and the software organizes the data in its local memory and apply computing. The output of a kernel function is also a data stream sent to the network by a DMA function. Moreover, the non-address Tx-Rx model is suitable for data broadcasting, especially when different target memories require different allocation patterns for their own computing algorithm. Broadcasting can save redundant data transfer in a CMP system [52].

\subsection{Star Network}

The Star network moves stream data between external memory and onchip distributed memories. The data being transferred on the Star network include the SIMD coprocessor program code and the computing input data and output data. The network architecture uses circuit switch based and non-address physical link to achieve short setup latency and power efficient data transfer. The Star network connects all SIMD coprocessors with the central multi-port DMA controller. The DMA controller has a dedicated communication link to every SIMD coprocessor. It is able to broadcast the same data on multiple links at the same time.

The non-address stream data transfer requires the on-chip memory address to be calculated at the SIMD coprocessor side. The computing kernel software determines the data allocation in the local memory. When transferring stream data for SIMD computing on VPE, the data can be permuted in the LVM during data transfer for conflict free parallel access. The VPE engine uses a SIMD architecture and parallel datapath to increase computing throughput. Previous studies show that the vector data shuffling and packing/unpacking operations limit the performance of conventional SIMD architectures [66]. ePUMA solves this problem by using software to control data allocation in the local multi-bank memory. The permutation ensures the VPE engine to access its required vector data in parallel and without bank-conflict during computation. 


\subsubsection{Data Streams}

The Star network handles communication tasks of loading kernels to SIMD and transferring computing data between main memory and SIMD local memories. The Star network communication models, including the addressing patterns and data types, are listed in Table 6.2. The kernel program contains two parts, the kernel scalar program and the kernel vector program. The kernel loading includes moving software program data from external memory to SIMD scalar program memory (SPM) and vector engine program memory (VPM). The SPM memory width is 32 bits, and the VPM data width is 128 bits. The transfer size is not limited by hardware and is determined by the kernel program size and data stream size. The memory addressing pattern for kernel program is sequential access for both source and target memories. For the access of computing data in the main memory, the most common addressing patterns in multimedia and communication applications are sequential, 1D stride and 2D stride. However, stride based access is not friendly to the system bus or the DRAM memory controller, both of them are in favor of transferring continuous data bursts. An alternative solution in ePUMA is to send normal burst requests from the central DMA controller to the external memory, but filter out redundant data when sending the data stream to the SIMD local LVM. 


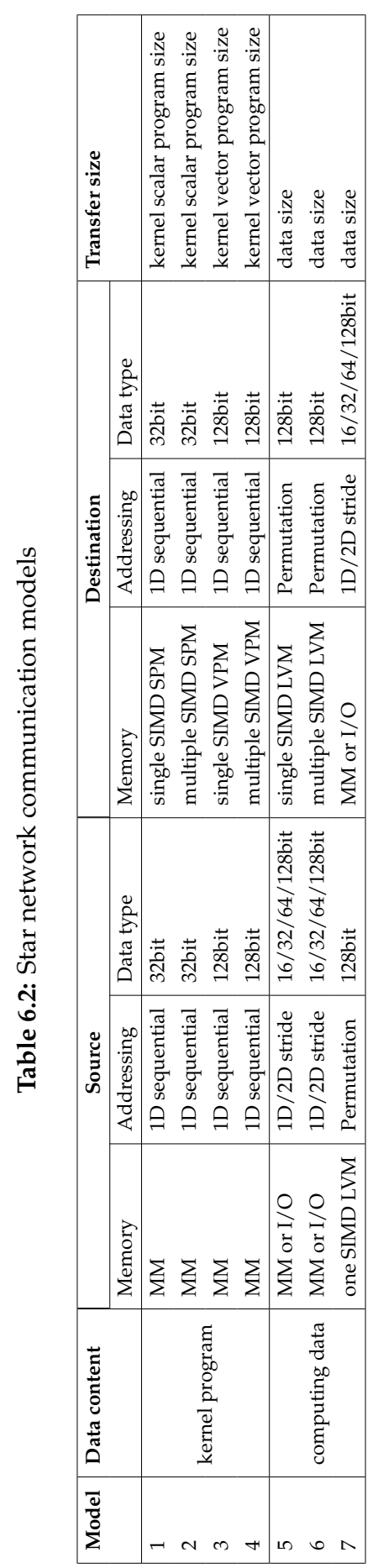




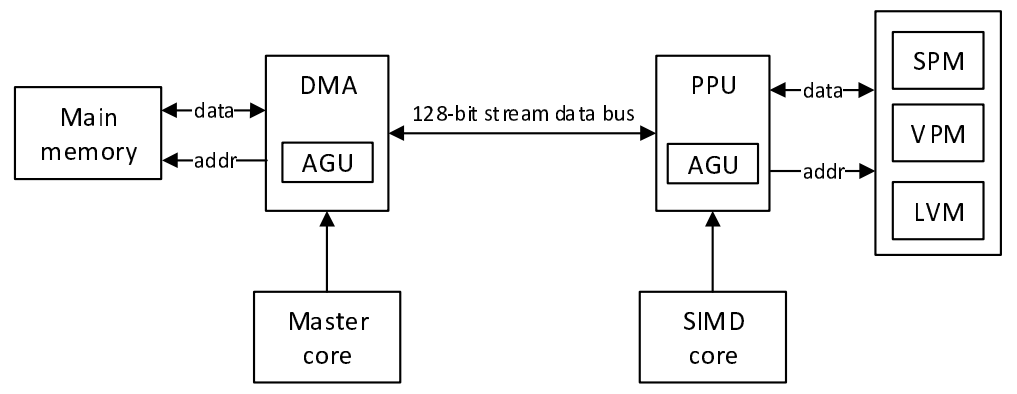

Figure 6.3: Communication link of the Star network

Broadcasting is a very common communication model in multiprocessor parallel computing. It is used to send the same kernel program from the software image stored in the external main memory to multiple SIMD coprocessors' onchip program memories. It can also be used to broadcast the same input data from main memory to multiple SIMD coprocessors' LVM memories. One application example using data broadcasting is the parallel matrix multiplication on multicore system [22], where all the SIMD coprocessors are executing the same kernel program, and one input matrix need to be broadcasted to all SIMD coprocessors.

\subsubsection{Hardware Architecture}

The Star network is optimized for the listed communication models in Table 6.2. The Star network uses a star topology. The master processor and a DMA controller are located in the star center. Each SIMD processor has a direct connection to the central node. All Star communications are controlled by the master processor. The Star network only supports data communications between central node and SIMD leaf nodes. Inter-SIMD communications use the Ring network without interrupting the master core. The Star network focuses on high bandwidth stream data transfer by using long data bursts, the design challenge is to reduce communication setup latency and hardware cost.

The physical link of the Star interconnection does not carry address 
information. As illustrated in Figure 6.3, the external memory address is generated by the central DMA controller. The address for SIMD local memories is generated locally at the leaf node. Only data stream is transferred on the network.

The central DMA controller is a multiport DMA controller. In conventional DMA controller design, the data addresses for source and target memories are all provided by the DMA controller using two separate address generation units (AGU). In ePUMA, the central DMA task configuration parameters only contain main memory addressing information. The AGU for SIMD local memories is distributed to each SIMD coprocessor. There are three major motivations of making this design decision. Firstly, it can be seen from Table 6.2 that different memories have different requirements on addressing pattern and data type. The main memory needs $1 D$ and 2D access of 16/32/64/128 bit data. SIMD local program memories (SPM and VPM) require simple sequential addressing of 32 and 128 bits data. The LVM accesses 128 bit data each cycle, views them as a vector of eight 16-bit words, and stores them in an 8-bank memory using LUT based permutation addressing. Secondly, during data broadcasting, same data block might be accessed by different SIMD processors in different ways; they need to apply different permutations on the input data stream. In this case, a distributed AGU approach is required. The third benefit is the saving of implementation cost and power consumption since no address bus is implemented on the Star network. The accesses to the local memories by the PPU and the SIMD core at a SIMD coprocessor's Idle and Busy states are listed in Table 6.3. The communications between the Star center and one SIMD's SPM, VPM, and LVM share the same 128-bit data bus, no concurrent data transfers to two of these local memories is needed.

The Star network transfers data packets. The packet header contains information for routing and control. In the downlink path from the central DMA controller to the SIMD coprocessor, this header contains message bits which indicate the data content. It is used at the SIMD side by the packet processing unit (PPU) to distinguish the data streams and de- 


\begin{tabular}{|c|c|c|c|c|c|c|}
\hline SIMD state & \multirow{2}{*}{ HW module } & \multicolumn{6}{|c|}{ Memory access } \\
\cline { 3 - 7 } & & SPM & VPM & VM-i & VM-ii & VM-iii \\
\hline \multirow{3}{*}{ Idle } & PPU & W & W & R/W & R/W & R/W \\
\cline { 2 - 7 } & SIMD core & - & - & - & - & - \\
\hline \multirow{2}{*}{ Busy } & PPU & - & - & R/W & - & - \\
\cline { 2 - 7 } & SIMD core & R & R & - & R/W & R/W \\
\hline
\end{tabular}

Table 6.3: SIMD local memory access requirements

liver them to the correct local memories. Another function of the packet header is to contain control command. A typical use case is after loading a kernel program the master wants to start SIMD execution. One way is to write to the start register. Another way is to create a data packet with a start command in the header and send it to the SIMD coprocessor by the central DMA controller. This packet contains only a start command and has no data payload. Using the DMA controller to send a control command is more efficient when the same command is broadcasted to multiple SIMD coprocessors, for example, when the master wants to start multiple SIMD coprocessors.

The uplink from SIMD to the central DMA controller is used for storing SIMD computing results in LVM back to the main memory. The header for uplink data packet contains a 32-bit address. The DMA engine can choose to use it as the base address for the main memory. It can also be configured to ignore this header field, depending on the application. Using this information from SIMD coprocessor, the DMA controller can work at the repeat mode and reduce the setup latency on updating the task parameters.

\subsection{Ring Network}

The Ring network moves data between LVM memories. It is only used for on-chip data communications. The architecture design of the Ring network includes the design of network topology, communication pro- 
tocol, and arbitration scheme.

\subsubsection{Data Streams}

The Ring network transfers only computing data on the chip between SIMD coprocessors. Two communication models on the Ring network are point-to-point communication and broadcast communication. The data stream on the Ring network is always 128-bit wide, which is the same as the LVM memory width. It does not need data packing or unpacking operation at the network interface.

The stream communication on the Ring network uses a message passing protocol. The sender issues a send task, and the receiver issues a receive task. Data is transferred when the two tasks are synchronized. The synchronization is done by hardware of the two PPU engines of the sender and the receiver. The physical link of the Ring network can choose to contain data buffer with configurable size, or use non-bufferable network. Applications can benefit from bufferable network if the inter processor data streams are short. The message passing protocol is suitable for predictable signal processing. It can minimize the overhead of interprocessor notifications using interrupts or mailbox communications.

\subsubsection{Hardware Architecture}

The main components of the Ring network are shown in Figure 6.4. It consists of two major blocks: the network channel control(NCC) and the network physical link(PHY). The NCC is responsible for network arbitration. The PHY module carries data streams during data transfer. The communication initiators and targets are connected to the network through the network interface (NI).

The datapath in the PHY module represents the network topology. The network is a circuit switch network. Its implementation is based on hardware multiplexers, which are controlled by the NCC through control registers. The network architecture provides a design framework, which allows customized topology in the datapath, and programmable arbitra- 


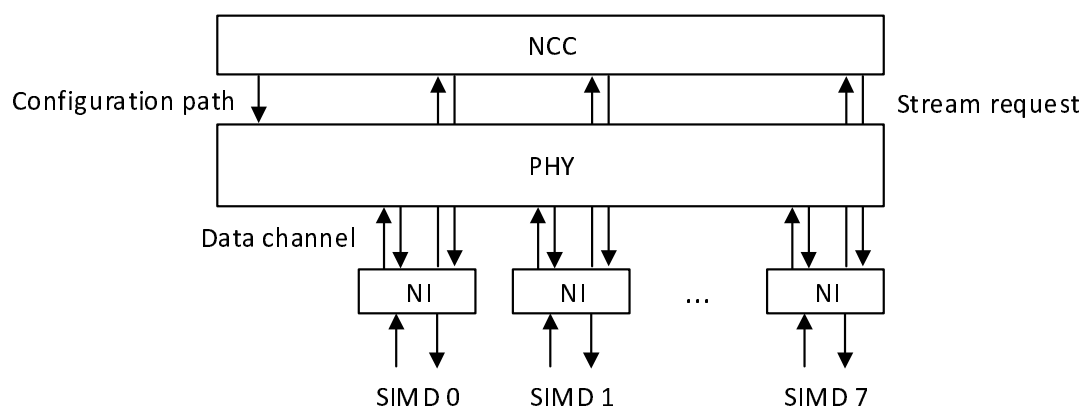

Figure 6.4: Ring network components

tion scheme in the control path. Figure 6.5 shows three typical network topologies that can be implemented under the framework. The circles in the figure represent network nodes, and the boxes represent processor cores. With the separation of control path and datapath, the same communication protocol can be used for different network topologies. The interface from the processor core to the network remains the same for different configurations.

The PHY module uses multiplexer based implementation. Inside each network node there are multiple multiplexers which route the data stream from input ports to output ports. Comparing the implementation cost and considering the peak concurrent communication bandwidth available on different network topologies such as crossbar, ring, and mesh network, the ring topology is a simple implementation with good performance for small core counts [67][68]. For ePUMA platform, the topology of multilayered ring is selected as the optimal solution. It provides high concurrent bandwidth at a relatively low implementation cost. The uni-directional physical link makes it easier for backend synthesis. Register slices or stages can be inserted when necessary to guarantee the clock speed. The same ring topology is also used in the STI Cell engine [8].

The control path in the NCC module handles the network arbitration 


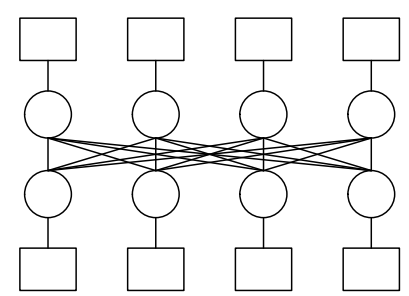

Crossbar

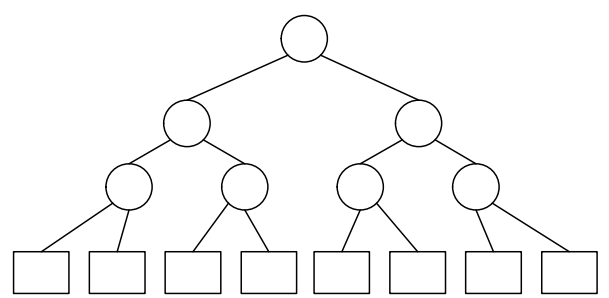

Tree network

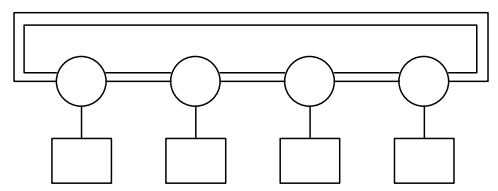

Ring network

Figure 6.5: Examples of PHY network topologies

and configures the network nodes in the PHY module to setup communication links. The NCC control flow includes the following four steps:

1. Receive send or receive requests from the NIs: It first synchronizes the send and receive tasks. If multiple receivers are selected by the send request, it waits until all the masked receivers to be in the receive state.

2. Setup the communication link in the PHY module: It selects the network nodes needed for the new link, waits until the current data transfers which occupies one or more nodes in the link to be finished, and then configures the nodes and locks the link for the new data transfer.

3. Grant the communication to the sender and the receiver: The acknowledge signal is sent to the sender and the receiver NIs. The data transfer starts.

4. Release the communication link after the data transfer: The last 
signal with the data stream indicates the end of a data transfer. This signal is passed to the NCC module to release the communication link and prepare the network nodes for the next stream.

There is a design tradeoff between the control overhead in the NCC and the hardware complexity in the PHY. If the PHY implements a full crossbar or a many layer ring, the second step in the NCC control flow of setting up the communication link is fast and has lower overhead, because there are more hardware resources to support more concurrent data streams.

The communication overhead is reduced by the stream level arbitration. As we can see from the control flow, the control path only operates on send or receive requests of data streams. When a communication link is setup for a stream, the data is transferred at full speed of 128-bit per cycle. The network doesn't support interruption of stream transfer. Once the transaction is started, the stream occupies the communication path in the PHY link until the last data beat is sent. The software programmer can decide the size of streams in order not to lock the PHY nodes for long data streams and block other data communications.

\subsubsection{Communication Protocol}

The communication protocol contains the Tx protocol for the sender and the Rx protocol for the receiver.

The Tx processor uses a FIFO interface to communicate with the network. The data sent to the network is in the form of a network packet with a header and a data payload. The data packet is prepared by the processor or the local PPU engine. The data packet can be blocked by the FIFO when sending data to the network. If the FIFO is full, the sender should not send more data to the network. The size of this FIFO at the network interface is configurable. For applications which transfer small data packets on the chip, it is beneficial to have the FIFO buffer large enough to keep an entire data packet, so that the computing at the processor core is not blocked. For applications which require large da- 
ta packets to be transferred between SIMD processors, it is not possible to store the entire data packet at the network interface. We can reduce the FIFO size to save network hardware. The package header contains information about the stream size and the destination processor ID. The destination node identification is used by the NCC to do arbitration and PHY configuration. The size information is used by the NCC for stream scheduling, it is also transferred to the receiver. In the case that the receiver doesn't know the size of input data and the work load, it reads the stream size from the PPU after the transaction and determine the number of local iterations to process the input data. The burst length of a data stream is not limited.

The Rx protocol defines the communication rule at the receiver processor. The receiver processor needs to follow the following procedure to receive data from network:

1. Configure the local AGU to receive data from network.

2. Set the ready flag to the network to show its ready-to-receive state.

3. Wait for data transfer.

The data channel signals from the network to the receiver processor include a data enable signal and a data bus.

\subsection{Serial Bus}

The Serial bus is designed for short latency mailbox communications between SIMD coprocessors. The high bandwidth Star and Ring networks are used to transfer data streams with unlimited packet length. To avoid interrupting long data streams or blocking the short message communication for a long and unpredictable time on the stream network, we use this dedicated serial bus with a minimum hardware cost to guarantee a fixed latency for short message communication. 


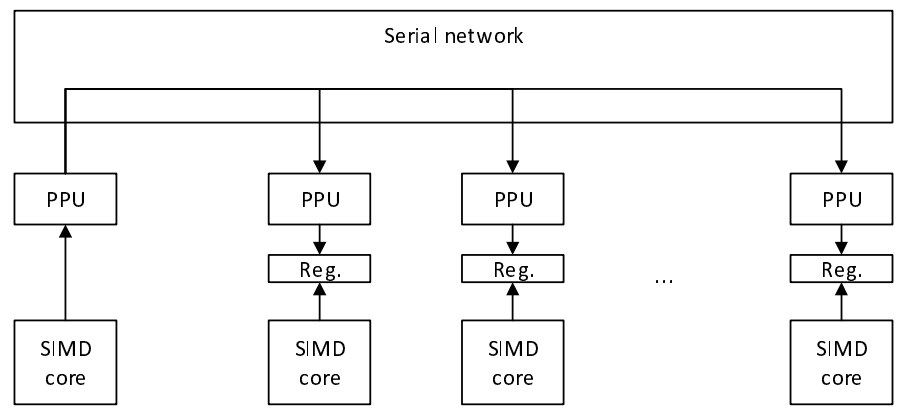

Figure 6.6: Serial bus communication

\subsubsection{Hardware Architecture}

Short message passing on Serial bus uses point-to-point interconnections. The communication link from one SIMD core to the other 7 SIMD cores is illustrated in Figure 6.6. The serial bus uses a single bit data bus and serial data transfer to reduce the hardware implementation cost. Each send request will send one 16 bit payload data through the serial bus to the target SIMD processor. The data packet on the serial bus contains an 8-bit field for selecting the target SIMD. The packet size of the Serial bus is 24 bit. During communication, the same data packet is broadcasted to all SIMD coprocessors. The PPU engine of each SIMD reads the packet header and decide whether to store the data into its local mailbox register or drop the packet. At the receiver side, the new packet will overwrite the old data in the mailbox. The packet transfer is not blocked by the interconnection or the receiver. Since dedicated point to point link is used, there is no extra transaction latency on arbitration or bus sharing. The transaction latency is fixed at 24 cycles. Data communication on the serial bus relies on the software to implement a handshaking protocol for inter processor synchronization. 


\subsubsection{Communication Protocol}

The hardware communication protocol of the Serial bus includes a protocol for the transmitter and a protocol for the receiver. At the transmitter side, it uses a send-and-forget protocol. The send command is issued by the processor core by writing to a special control register in the PPU engine, which then prepares the data packet and send it to the serial bus immediately. The sender does not wait for the data to be delivered.

At the receiver side, there are seven mailbox registers to store the received data. Each register has one flag bit to show the state of the corresponding register. The state bit is set as valid when new packet from the serial bus arrives. It is cleared by the read operation from the local SIMD processor. Newly arrived data will overwrite the old data even if it is not read by the local processor. The software simulator will report the overwriting to previous valid data as an error operation.

\subsection{DMA Controller}

The central DMA controller of the Star network is used to move data between external memory and on-chip SPM memories. The data moved by DMA controller include SIMD kernel programs and stream data. To reduce the DMA setup latency, the DMA controller separates task configuration from data transaction. It supports multiple tasks to be configured and stored in a task memory. The master controller can configure the next DMA task while the DMA is executing the current transfer. Continuous tasks in the task memory can be linked together and completed in one DMA transaction.

\subsubsection{Task Configuration}

Table 6.4 lists the configuration parameters of one DMA task. The first configuration register (16 bits) has three subfields used to specify the source memory, the target memory, and the SIMD selection. The source and target memory can be one of the main memory(MM), SPM, VPM, 


\begin{tabular}{|c|c|c|c|c|}
\hline Reg. & Parameter & Bits & Field & Description \\
\hline 0 & $\begin{array}{l}\text { source } \\
\text { target } \\
\text { simd select }\end{array}$ & 4 & $\begin{array}{r} \\
{[11: 8]} \\
{[7: 0]}\end{array}$ & $\begin{array}{l}\text { source memory: } 0=\mathrm{MM} \text {, } \\
1=\mathrm{SPM}, 2=\mathrm{VPM} 0,3=\mathrm{VPM} 1, \ldots, \\
0 \times \mathrm{F}=\mathrm{LVM} \\
\text { target memory } \\
\text { select one or multiple SIMD } \\
\text { cores for data transfer }\end{array}$ \\
\hline 1 & data type & 3 & [2:0] & $\begin{array}{l}\text { data size of single data ele- } \\
\text { ment, } 8 / 16 / 32 / 64 / 128 \text { bits }\end{array}$ \\
\hline 2 & size & 16 & {$[15: 0]$} & $\begin{array}{l}\text { total number of data elements } \\
\text { to transfer }\end{array}$ \\
\hline 3 & base address & 32 & [31:0] & $\begin{array}{l}\text { the start address of the first el- } \\
\text { ement in the system memory } \\
\text { map }\end{array}$ \\
\hline 4 & x-step & 16 & {$[15: 0]$} & $\begin{array}{l}\text { horizontal stride in the num- } \\
\text { ber of bytes }\end{array}$ \\
\hline 5 & x-size & 16 & [15:0] & $\begin{array}{l}\text { number of data elements in a } \\
\text { row }\end{array}$ \\
\hline 6 & y-step & 16 & {$[15: 0]$} & $\begin{array}{l}\text { vertical stride in the number of } \\
\text { bytes }\end{array}$ \\
\hline 7 & ready & 1 & [12] & $\begin{array}{l}\text { if enabled, DMA will send a } \\
\text { interrupt to the master core } \\
\text { when the task is finished } \\
\text { the configuration ready flag, } \\
\text { set by master, cleared by DMA } \\
\text { controller } \\
\text { link to the next task. 0: not } \\
\text { linked, } 1 \text { : linked }\end{array}$ \\
\hline
\end{tabular}

Table 6.4: DMA task configuration parameters 
or LVM. In each DMA task configuration, one of the source or the target memory should be the MM, because the central DMA controller only handles data communication with external memory. The SIMD selection field uses 8 bits to mask eight SIMD coprocessors. When MM is the source memory, it can select multiple SIMD cores as destinations to broadcast data. If the MM is the target memory, the source memory can only be the LVM memory from one SIMD. This is because we do not transfer kernel program code from the on-chip SPM memory back to the main memory. The remaining communication patterns are illegal DMA tasks and will result in undefined behavior.

As can be seen from the parameter list, the DMA task only contains addressing information (register 2-6) for the main memory. The addresses of the distributed SPM memories during data transfer are generated by the PPU engine. The main memory data access uses five parameters to support $2 \mathrm{D}$ addressing of up to 128 bit wide data elements. The data type specifies the basic data transfer size when the DMA controller sends request to the system bus. The smaller width data type should only be used when there is a stride between neighboring elements. Continuous data should use the 128-bit data type to minimize access latency. Register 2 specifies the total number of data elements to be transferred in this DMA task. The size in bytes is determined by the number of elements and the data type. The base address uses 32 bit unsigned data to specify the physical address of the first element. The three addressing parameters, $x$-step, $x$-size, and $y$-step, are used to define the shape of the 2D data array. The two stride values $x$-step and $y$-step are 16-bit signed values to support both forward and backward jumping. The $x$-size specifies the number of data elements in one row. The last register contains two flags, ready and link. The ready flag is written by the master core at the end of a task configuration to indicate that all the parameters of the current task have been written. The DMA controller looks for this ready bit to start processing this task. The link parameter is used to link multiple DMA tasks together and execute them by one DMA start command. The two flags are cleared by the DMA engine after the parameters are loaded. The 


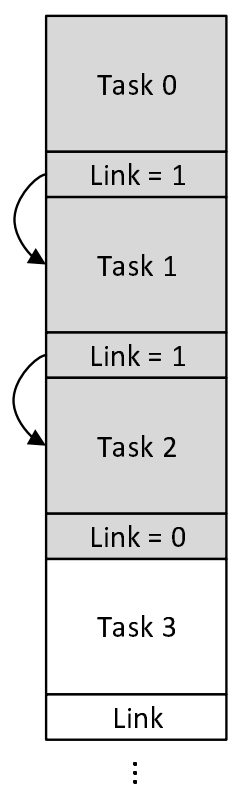

Figure 6.7: Link of DMA tasks

implementation and operation of the hardware task queue using the two flags are explained in the following section.

\subsubsection{Hardware Task Queue}

The DMA controller contains a task memory to store multiple DMA tasks, as shown in Figure 6.7. It support overlapping task configuration operation with DMA execution, and linking of multiple tasks into one transaction. These two features can reduce task configuration overhead and control overhead in finishing one task and start the next task [52].

The access to the task memory depends on the implementation. The task memory can use multiport register file or on-chip SRAM as data storage. The master core needs read-write access to the task memory, and the DMA controller needs read access to get configuration parameters. For a small-sized task memory, for example a memory for less than 16 tasks, it is better to use register file to store DMA tasks. The DMA con- 
troller can use the hard-wired parameters directly without task loading overhead. If the task memory is designed to store many tasks, the hardware cost of a register file is too high. The DMA controller should use a descriptor memory based architecture [69], which uses a shared memory or a dedicated SRAM to store descriptors of DMA tasks. A descriptor is a data structure that contains configuration parameters and control information for chaining of multiple tasks, similar to the control register parameters. It can even use data pointers to link multiple tasks. In this case DMA tasks are not necessary to be stored in continuous memory spaces. More software flexibility for task chaining and larger storage space are advantages of this approach. The cost is the task loading overhead to read out parameters from the memory.

Multiple DMA tasks can be linked with its neighboring tasks to form a task queue. The link flag in a task configuration indicates that after the current task, the DMA engine continues with the next task automatically, without interrupting the master core. In our design, the last DMA task is linked to the first task at the beginning of the task memory to form a circular task buffer. Software can manage the task queue at runtime by using the ready flag.

A common operation mode is to divide the task memory into small sections, each section contains one or multiple tasks. All the tasks in one section are executed by one DMA transaction. The last task in a section has its link flag disabled, so that the DMA engine will stop after finishing this task. The transaction of a section of DMA tasks is started by giving the number of the first task in the section when sending the start command.

\subsubsection{DMA Control Flow}

The control registers are separated from the task memory in implementation. The registers are listed in Table 6.5. It contains both command registers to start a DMA transaction and status registers to monitor the state of DMA engine. The RESET control register will perform a software reset 


\begin{tabular}{|c|c|c|c|c|}
\hline Addr. & Reg. & Bits & Field & Description \\
\hline 0 & RESET & 1 & [0] & software reset \\
\hline 1 & START & 4 & [3:0] & $\begin{array}{l}\text { start the DMA, the value is the first } \\
\text { task in the task memory }\end{array}$ \\
\hline 2 & STATE & 2 & [1:0] & $\begin{array}{l}\text { the state of DMA engine: } 0=\text { IDLE, } \\
1=\mathrm{LOAD}, 2=\mathrm{TRANS}\end{array}$ \\
\hline 3 & TASK & 4 & {$[3: 0]$} & the current or last DMA task \\
\hline 4 & BYTES & 32 & {$[31: 0]$} & $\begin{array}{l}\text { the total number of transferred data } \\
\text { since the DMA is triggered. }\end{array}$ \\
\hline
\end{tabular}

Table 6.5: DMA control registers

of the DMA engine, disrupt the ongoing task, clear the internal buffers, and switch the DMA engine to the IDLE mode. If the task memory is implemented by registers, it also zero-resets the task memory. The write to the START register has two effects, it sends a one cycle trigger event to start the DMA engine, at the same time, the write value is kept in the register which is read by the DMA engine to find the first task to load from the task memory. The other three registers are status registers which are read-only by the master core. By reading these status registers, the master core can check the DMA status including the current state of the data transfer state machine, the current executing or the last executed DMA task number, and the total number of bytes that have been transferred in this transaction, including all data in the linked tasks.

The software can manage DMA tasks in two ways, using the task queue method or the section based method.

\section{Task queue based control flow}

In the task queue management, the DMA engine never stops after start. It always checks the next task in the queue to execute by polling the ready flag. The following steps should be followed by master software to enqueue a task: 
1. Check the top task buffer availability by polling the flag register. Wait until the flag register is cleared by the DMA engine.

2. Write new task parameters into the top task buffer.

3. Write the last flag register with ready $=1$ and link $=1$.

4. Update the top task pointer.

\section{Section based control flow}

The second flow is used for the section-based task memory management. The transactions are not managed in queues. The DMA engine stops after finishing the last task in a link. The software can only start a new task after the previous task has been finished. Since the task memory can store multiple task configurations, we can configure the next task using alternative task buffers while the DMA engine is transferring data for the current task. A task buffer can be used for new configurations when the ready bit is cleared by the DMA engine, which indicates that the task has been loaded by the DMA engine. After the current DMA transaction is finished, the new task can be started immediately with no configuration overhead. The control flow for the section-based management is concluded as below:

1. Configure task using spare task buffers.

2. Read DMA status register and wait until it finishes the current task.

3. Write the start task number to the START register.

The DMA engine contains a state machine to process a transaction request in the following steps:

1. The engine (in IDLE state) is started by a Start signal.

2. It gets the first task ID number from the start register.

3. It synchronizes with the ready flag of the current task. 


\begin{tabular}{|c|c|c|}
\hline HW. Unit & Inst. & Description \\
\hline SC & 1 & 32bit scalar core with $\mathrm{C}$ compiler support \\
\hline VPE & $\geq 1$ & $\begin{array}{l}\text { 128bit vector core, an independent processor that } \\
\text { runs VPE SIMD instructions }\end{array}$ \\
\hline PPU & 1 & local communication engine \\
\hline $\mathrm{ACC}$ & $\geq 0$ & optional hardware accelerator \\
\hline
\end{tabular}

Table 6.6: SIMD coprocessor processing units

4. The engine is in LOAD state to load configuration parameters. It creates a local copy of the task, and then clears the ready and link flags.

5. The DMA engine is in TRANS state to transfer data from source memory to target memory.

6. When the transaction is done, DMA checks the link flag. If it is enabled, it continuous to process the next task automatically from step 3 .

7. DMA changes back to IDLE state, and the other status registers are updated according to the transaction.

\subsection{SIMD Local Memory}

Each SIMD of ePUMA is a processor subsystem that consists of computing units, communication engines and local memory modules. It uses the transitioned memory architecture to move local data between computing units and communication engine.

\subsubsection{Processing Units and Scratchpad Memories}

The processing units in each SIMD coprocessor are listed in Table 6.6, which include one 32bit scalar controller (SC), one or multiple 128bit vector processing engines (VPE), one PPU data transfer engine, and option- 


\begin{tabular}{|c|l|l|}
\hline Mem. module & Width & Description \\
\hline SPM & 32 bit & scalar core program memory \\
VPM & 128 bit & VPE core program memory \\
LVM & 16 bit $\times 8$ & 8 banks parallel data memory \\
\hline
\end{tabular}

Table 6.7: SIMD coprocessor local memory modules

al hardware accelerators (ACC). The execution of VPE, PPU and ACC is controlled by the SC core. The PPU engine is responsible for stream data communications with the Star and Ring network, and short message communication using the serial bus.

SIMD local memory system contains three types of SPM memories, as listed in Table 6.7. These local memories are not mapped to the global memory space. They can only be accessed by the local processing units for computing or communications. The SPM is a 32-bit wide memory which serves as the program and data memory for the scalar controller. It keeps the instruction code and stack of the scalar program. The VP$M$ stores vector instructions for VPE execution. Both the SPM and the VPM program memories are initialized by kernel loading function of the master program. The LVM memory is local data buffer for stream data. Each LVM module contains 8 banks of 16-bit single port memories to provide 128-bit parallel data access for SIMD computing. The number of LVMs in one SIMD coprocessor is configurable in the hardware platform, depending on the number of VPEs and ACCs. The default configuration contains three LVMs. At runtime, two LVMs are connected to the VPE for computing, and one LVM is connected to the PPU to transfer data. The input and output data streams are transferred by the local PPU engine through the on-chip network.

The SIMD local memories (SC, VPM and LVM) receives data access requests from the computing modules (SPE, VPE and VCC) and the communication module (PPU). The access to the program memories (SPM and VPM) uses a static arbitration based on the SIMD state. The access to LVM memories is switched dynamically by the SIMD scalar controller. 


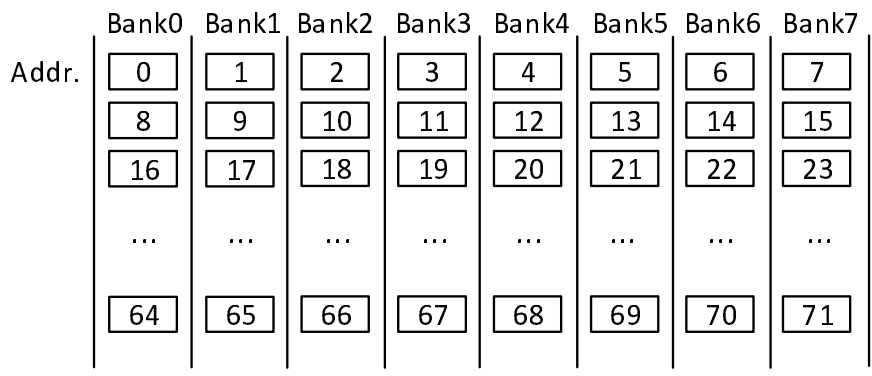

Figure 6.8: LVM multibank memory address mapping

The SIMD coprocessor has two states, IDLE and BUSY. In the IDLE state, the processing units are stopped and has no memory access. The PPU engine is granted write access to the program memories SPM and VPM to load kernel program from the Star network. The LVM switch and PPU engine can be controlled by the master core by setting the access control register master_control, which changes the access to the SIMD control register between master core and SIMD local controller. In the IDLE mode, the master can have full control of the SIMD local memory system and perform data transfer to and from all the LVM memories. In the BUSY mode, the SC core executes software program from the SPM. It controls the execution of VPE and ACC. The SC core accesses SPM for program data. The VPE core accesses VPM for vector program. At runtime, the PPU, VPE and the SC scalar controller connect to different LVM memories. Data communication and computing tasks operate on different LVM memories at the same time.

The address map of LVM uses interleaved addressing as shown in Figure 6.8, address 0 in bank 0 , address 1 in bank 1 , and etc..

\subsubsection{Transitioned LVM Buffers}

The data movement between processing units inside a SIMD coprocessor uses a technique called transitioned memory buffers [45]. The ownership of a LVM buffer is switched from one processing unit to another, but the 


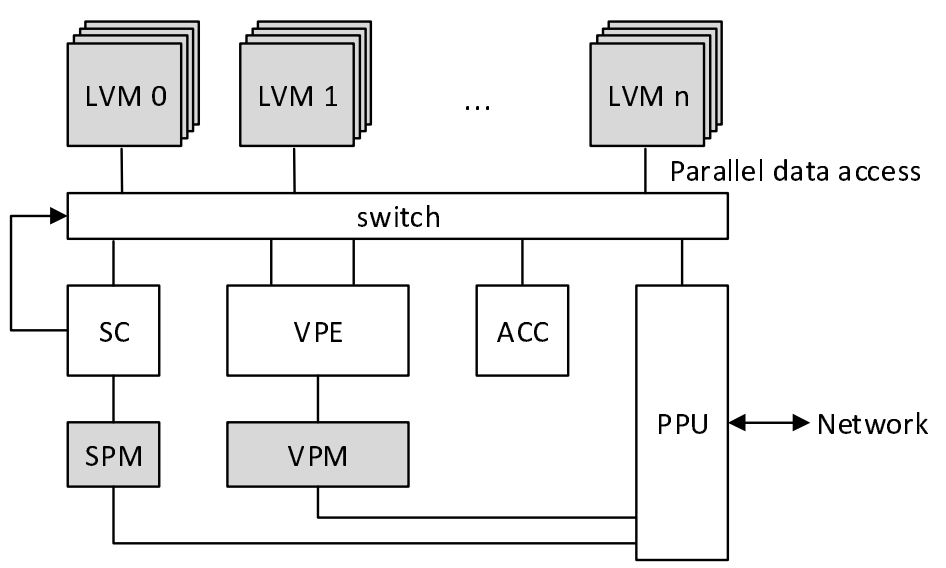

Figure 6.9: SIMD coprocessor local interconnection through the LVM switch

contents of the LVM memory do not transfer. The connection between memory modules and processing units are shown in Figure 6.9. The LVM data memories are connected to the processing units (SC, VPE, PPU, ACC) through a hardware switch which performs the memory transition. The LVM switch is controlled by the SC core through control registers. The connectivity of two program memories SPM and VPM are switched by the SIMD state, idle or busy. The SIMD coprocessor is in idle mode when it is not started by the master or after the SC core executes a STOP instruction. If the core is started or re-started after stop, it is in the busy mode. In the idle mode, the SPM and VPM are connected to PPU for kernel loading. In the busy mode, the SPM and VPM are connected to the SC core and VPE core as program memory. The master can not load kernel to SIMD when it is in busy mode.

\subsubsection{SIMD Accelerator Extension}

Each SIMD processor can be extended with hardware accelerators. The accelerator processes data in the LVM, under the control of the SC core. This part defines the accelerator interface for connecting an accelerator to the SIMD local memory system. The interface includes two groups of 


\begin{tabular}{|c|c|c|l|l|}
\hline Addr. & Name & Bits & Type & Description \\
\hline 0 & RESET & 1 & Normal & software reset \\
1 & START & 0 & Write trigger & start trigger, one cycle \\
2 & STATE & 32 & Normal & status register \\
$3-15$ & PREGS & 32 & Normal & parameter registers \\
\hline
\end{tabular}

Table 6.8: SIMD coprocessor accelerator's control register interface

signals, the control interface and the data interface. The control interface consists of several control registers which are mapped to the SC core's memory space. The SC core reserves 16 address space for each accelerator. The local address map and register type are illustrated in Table 6.8. The RESET, START, and STATE registers are general control registers to perform software reset, start trigger, and monitor accelerator status. There are 13 addresses reserved for custom accelerator parameters.

The data interface to the LVM switch uses parallel single port memory interface. LVM is a parallel memory consists of 8 memory banks, each memory bank is 16 bit wide. It supports up to eight 16 bit vector data access in one cycle. An accelerator can choose to implement a memory interface of $16 \times \mathrm{N}$ bits $(1 \leq \mathrm{N} \leq 8)$, depending on the throughput of its datapath. The address bus should provide addresses of each 16bit. It is the accelerator's responsibility to guarantee that the addresses doesn't contain bank conflict. Accelerators can take advantage of parallel memory access by specifying the input data allocation in the LVM. Then the master core will control the PPU to shuffle the input data according to the accelerator's access patterns. The data interface signals are listed in Table 6.9.

The logic timing of the data interface to the accelerator contains two cycles delay. The memory data is registered twice, one cycle for the memory module, another cycle for the LVM switch and shuffle network. The data comes two cycles after the address is presented on the interface. 


\begin{tabular}{|l|c|l|}
\hline Signal name & Bits & Description \\
\hline CS[7:0] & 1 & memory bank enable \\
\hline WE[7:0] & 1 & write enable for eight memory banks \\
\hline ADDR[7:0] & {$[15: 0]$} & vector address \\
\hline RDATA[7:0] & {$[15: 0]$} & read vector data \\
\hline WDATA[7:0] & {$[15: 0]$} & write vector data \\
\hline
\end{tabular}

Table 6.9: SIMD coprocessor accelerator's LVM interface

\subsection{Stream Communication Scheduling}

The stream data transfer on the Star and Ring networks requires the cooperation between DMA and PPU engines to read a bulk of data from the source memory, send them to the network, receive data from the network and write them into the target memory. Both the DMA and PPU engines have a dedicated hardware task queue. Multiple tasks in the queue can be executed after one start command without interrupting the controller by using the task link mechanism. For predictable streaming applications, the stream communication tasks are scheduled statically.

Figure 6.10 shows an example of static scheduling of Star and Ring communication tasks. In the example, there are four Star network communication tasks T0, T1, T2, T3. The corresponding DMA and PPU tasks from $\mathrm{T} 0$ to $\mathrm{T} 3$ are shown in the queue of each device. In addition, the PPU queues of SIMD0 and SIMD1 have two on-chip stream communication tasks T4 and T5 for data movements through the Ring network. The data transfer engines at the transmitter and the receiver performs synchronization in hardware. It is not necessary to use any inter-core notification method to synchronize for communication. It can be seen from the example that careful scheduling is necessary to avoid deadlocks in the network. For example, in this case, if SIMD1 schedules task T5 before $\mathrm{T} 4$, a deadlock will occur. 


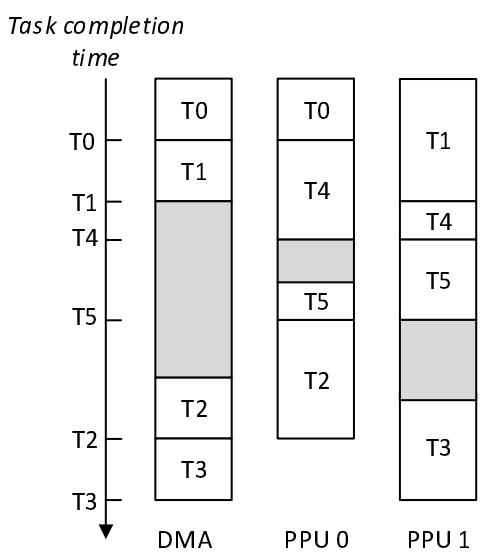

Figure 6.10: An example of Star and Ring communication static scheduling

\subsection{Summary}

This chapter introduces the ePUMA memory subsystem architecture. It describes the memory hierarchy, the multi-layer interconnection network, the multi-port DMA controller design, and the SIMD local memory subsystem. The memory hierarchy uses on-chip SPM memories to hide external memory access latency. It relies on software to explicitly control DMA transactions to move data between memories. The data movement is through a multi-layer network which consists of the Star and Ring stream networks and a serial bus. The Star network is responsible for data communication with external memory. The Ring network transfers data between on-chip memories. The Serial bus is dedicated point-to-point interconnection which transfers 16-bit data messages between SIMD coprocessors. The main component of the Star network is the central DMA controller. It uses task descriptors and DMA links to reduce configuration overhead and task switching overhead. The SIMD local memory system connects multiple processing units and communication units with the SPM memories, including two program memories and multiple LVM memories. The SIMD coprocessor is extendable by adding VPE vector engines and ASIC hardware accelerators. 


\section{Chapter 7}

\section{Software Development and Toolchain Support}

This chapter describes the contributions in the development of ePUMA software toolchain. It includes software modeling of the memory subsystem components and the master controller, and the integration of the multicore system simulator. It also includes the implement of the software library functions and a multitasking framework. Other works such as the VPE simulator and the ePUMA tools from other work packages of the ePUMA project are included in this chapter to make a complete description of the ePUMA software development environment.

The software development for ePUMA requires both parallel programming for multicore and kernel programming of SIMD processors. The software programmer needs to handle data movements between scratchpad memories. ePUMA software contains a hierarchy of software code. The master code is the top level control code. The SIMD scalar code performs local control of a SIMD coprocessor. The SIMD VPE code is the lowest level computing task, which focuses on stream data computing. The execution of VPE tasks is under the control of SIMD scalar core. The SIMD scalar code and the VPE tasks running on the same SIMD coprocessor form one SIMD kernel. The master core manages the kernel execution on different SIMD coprocessors by loading kernel programs to the 
coprocessors, feeding them with input data, and collecting the computing results. Inter-kernel communication is possible during kernel computing and without the master's intervention. The programming model uses the kernel based parallel programming [70].

ePUMA platform provides a software toolchain and library functions for software development. The basic tools include a cycle accurate simulator, scalar core compiler, SIMD assembler, and a linker tool to combine the control code and kernel code into one binary. High level tools are under development, such as kernel identifier and auto-vectorization. This chapter will introduce the software development flow and the software project organization. It will also discuss about a multitasking framework for ePUMA.

\subsection{Kernel Based Parallel Programming}

ePUMA uses a kernel-based parallel programming model. The master controller executes the main program. It sends kernel tasks to different SIMD processors to execute in parallel. Conventional DSP processors are mostly programmed in $C$ language and complementing the computing intensive functions by assembly subroutines to achieve the greatest computing performance. ePUMA software is developed in a similar way, the system software and the control code are programmed in $\mathrm{C}$ and the computing kernels are programmed in SIMD assembly code. The difference is that the assembly subroutines are executed by parallel SIMD coprocessors, and multiple such subroutines for different tasks can execute in parallel on different SIMD processors. After issuing a kernel on one SIMD, the master can move to another task, for example send a second kernel to another SIMD, and come back to respond when the first kernel is finished. 


\subsubsection{Master Program}

To work with kernels in the main program, the master software uses libray functions to control kernel execution. The master core is programmed in standard C. The master library (Appendix A) includes library functions to control the memory subsystem and SIMD coprocessors. An example of a master program is shown in Listing 7.1.

Listing 7.1: Master code example

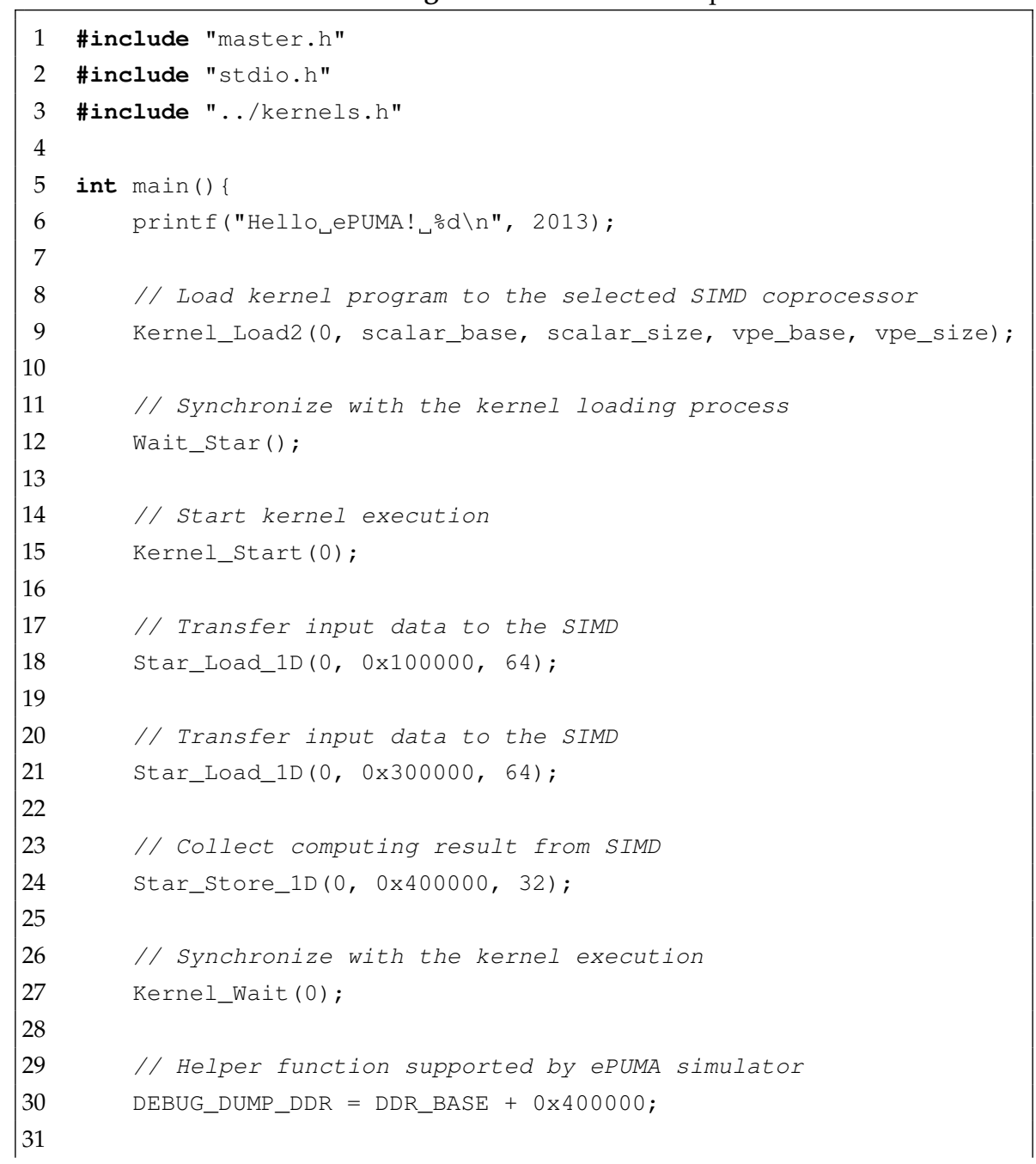




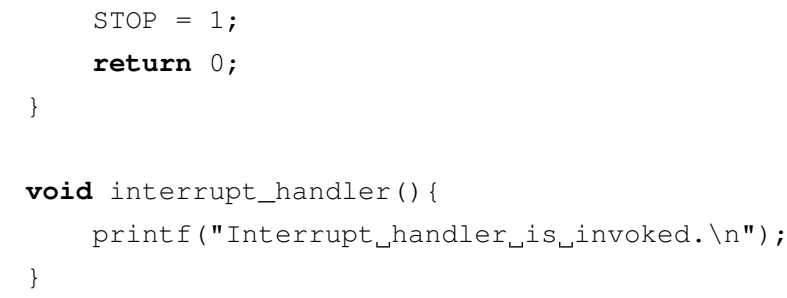

The DMA functions control the data movements on the Star network. It handles data transfer between off-chip memory and SIMD local memories. In this example, the function Kernel_Load2 loads the pre-compiled kernel to SIMD 0. The kernel contains two parts, the scalar code located in the main memory at address scalar_base, and the VPE code located at vpe_base. The base and size parameters are generated by the linker tool which packs all binaries into one software image. The kernel parameters are stored in the header file kernels.h (line 3) generated by the linker. The DMA data transfer is synchronized by the function Wait_Star.

The SIMD coprocessor control library functions control the execution of kernel tasks. In the example, the function Kernel_Start (0) starts the execution of SIMD 0. The function Kernel_Wait (0) synchronizes with the kernel execution on SIMD 0.

The master library also provides runtime debug functions such as DEBUG_DUMP_DDR for debug purpose. Programmers can insert those function to monitor execution flow, dump memory data, and verify system configurations from the platform simulator.

\subsubsection{Kernel Scalar Control Code}

The kernel control code runs on the scalar controller in SIMD. It is also programmed in standard C. The code is loaded to SPM memory by the master core. The SIMD scalar program controls the LVM switch and the PPU to send or receive data through the network. It also controls the execution of the VPE task. The SIMD scalar controller library (Appendix B) provides API functions to perform the control operations. Listing 7.2 
shows an example of kernel scalar code.

Listing 7.2: SIMD scalar code example

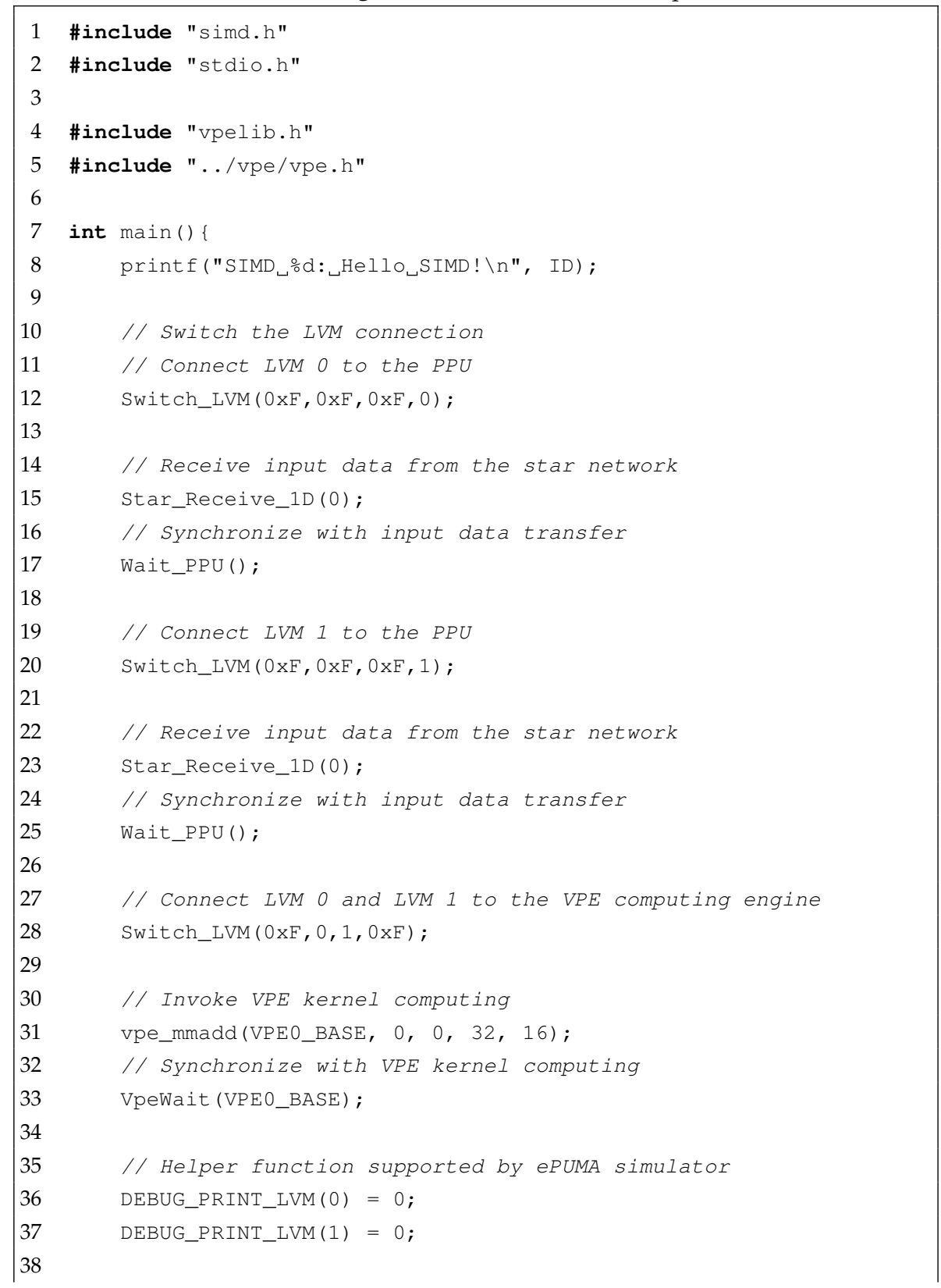




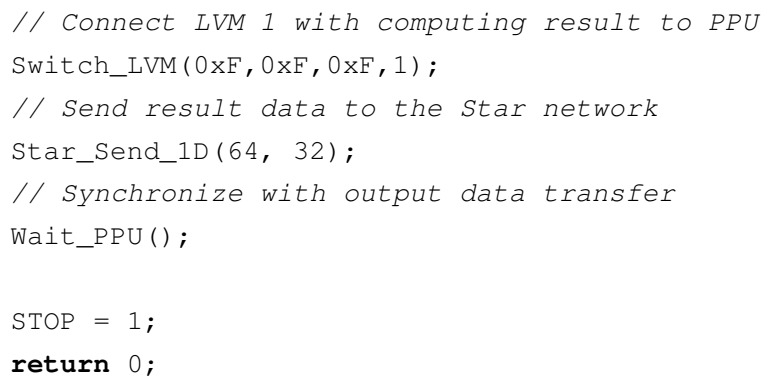

In this example, the library function Switch_LVM switches the LVM connections to the memory ports of SC core, VPE core, and PPU. The VPE core has two LVM memory ports, and each of the SC core and the PPU engine has one LVM port. The four parameters of the Switch_LVM function specifies the LVM connected to these ports. Starting from the left, they are the SC port, VPE $\mathrm{m} 0$ port, VPE $\mathrm{m} 1$ port, and PPU port. The value $0 \mathrm{xF}$ means this port is disconnected from LVM memory.

The scalar library functions Star_Receive_1D ( 0 ) and Star_Send _1D $(64,32)$ controls the PPU engine to transfer data streams through the stream network. The PPU task is synchronized by the function wait_ PPU. As we can see from the code, the software programmer needs to control the data transfer and the memory switch. The goal is to overlap the computing and communication. Advanced PPU functions can include data permutations in the transaction, which shuffles data in the LVM memory for conflict free parallel access by the VPE engine.

The VPE task is started by a wrapper function, such as vpe_mmadd in line 22 of the example. This function passes parameters to the VPE engine and starts the execution. The function VpeWait synchronizes with the VPE task. In ePUMA software program, each VPE task has a C wrapper function to start the execution from the scalar controller.

Debug functions similar to the master program's are supported in the SIMD scalar program. Programmers can use the runtime debug functions to print debug information on the simulator terminal, or dump memory values to text files, such as the DEBUG_PRINT_LVM $(0)$ func- 


\begin{tabular}{|l|c|}
\hline Algorithm & Number of instructions \\
\hline Complex matrix mul. & 14 \\
8-tap real FIR filter & 10 \\
48-tap complex FIR filter & 27 \\
Radix-2 FFT transform & 70 \\
Radix-4 FFT transform & 56 \\
\hline
\end{tabular}

Table 7.1: VPE kernel code size [65] C)2010 IEEE

tion in the example.

\subsubsection{Kernel SIMD code}

The software for the VPE is programmed in assembly. The VPE computing engine executes vector instructions to do SIMD computing. There are two reasons why we choose to use assembly programming for the kernel SIMD code. The first is to get the greatest performance of the VPE engine by programming at a low level. Secondly, today's compiler techniques can not show satisfactory performance for software vectorization. This is especislly true for the ePUMA architecture with more complex vector operations than conventional SIMD architectures. The details of the VPE engine and SIMD instruction set is another PhD thesis work in the ePUMA project. This dissertation only gives examples for illustration.

The SIMD instructions use long instruction word of 64-bit for base instructions and 128-bit for advanced instructions. The SIMD code size is usually small. Table 7.1 lists typical VPE tasks and the number of instructions in the program. These typical DSP kernels are programmed with less than 100 instructions.

Below is a SIMD data copy instruction to demonstrate the software programmable data permutation in the LVM memory. 
Listing 7.3: SIMD kernel program example of data permutation

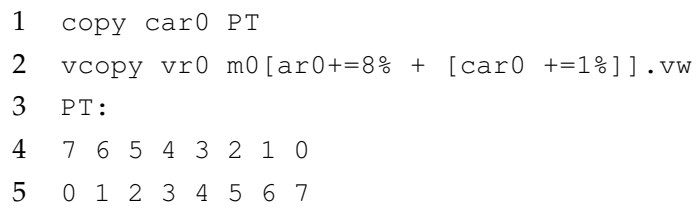

The software programmer can use the LUT to develop specific addressing patterns. In the vcopy example, $\mathrm{mo}$ is a memory port connected to one LVM through the switch. Which LVM memory is connected to the VPE is controlled by the scalar controller by calling the LVM switch function. The access pattern of $\mathrm{m} 0$ is specified in the instruction, which has two parts, the $\operatorname{ar} 0+=8 \%$ controls the scalar branch in the vector $\mathrm{AGU}$, and the $\mathrm{cm}[\operatorname{car} 0+=1 \%]$ configures the vector branch of the offset address of each data element. The PT is a label which refers to a data section in the program memory where the permutation table is stored. In this example the permutation table contains two entries as in line 4 and 5 .

\section{2 ePUMA Simulator}

This section describes the design of the architecture simulation model. The simulator can run in command line, it also provides software interface to work with graphics frontend or other tools like Python and Matlab.

\subsubsection{Simulator core}

The simulator core is developed in $\mathrm{C}++$. It uses the modular property of the object oriented language to model the hardware architecture. In the simulator each hardware module is modeled as a $\mathrm{C}++$ class. It contains submodules as class members. Each class has one simulation function cycle () to simulate the behavior when the clock is increased by one. The constructor of the module initializes the submodules and setup the connection of signals between submodules. Listing 7.4 shows an exam- 
ple of the memory subsystem top module.

Listing 7.4: Simulator modeling example

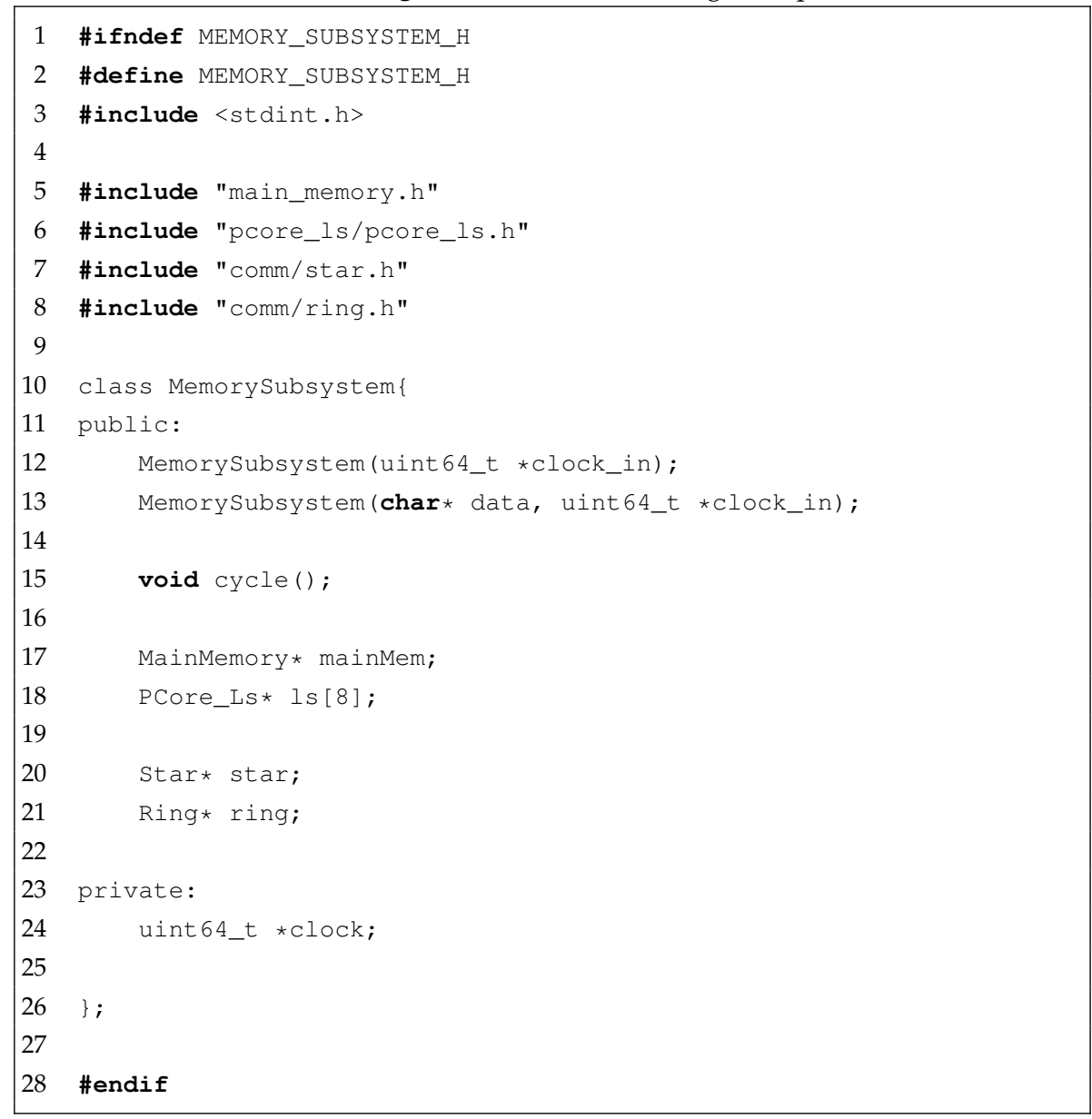

In the above example, the module MemorySubsystem contains submodules of one global memory Ma inMemory, eight SIMD local memory modules PCore_Ls, and two stream networks Star and Ring. Two constructors are provided. The second constructor can initialize the main memory by the data input. The cycle () function is called from the upper level module for each clock cycle during simulation. The implementation of this cycle () function of module Memorysubsystem is listed in Listing 7.5. As we can see from the code, it issues the cycle 
functions of its submodules. The main memory is a slave device so it does not contain a cycle function.

Listing 7.5: An implementation example of the cycle function

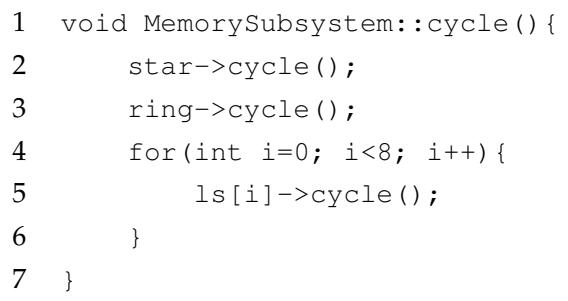

The ePUMA simulator is built using this module based framework. The simulation models of the processing units, including the master core, the SIMD scalar controller, and the SIMD VPE computing engine, are designed separately and integrated with the memory subsystem simulator. The memory subsystem model provides all the program and data memories for these processor cores.

\subsubsection{Simulator interface}

The ePUMA multicore system simulator can run in command line mode. It also provides software interface to connect to other environments such as GUI, Python script, and Matlab. The software interface is built using the socket IO of the host computer. When the simulator is started in this mode (the remote mode), the simulator core starts a socket server to wait for the client to be connected. The client can be one of the GUI, script, or Matlab software, which has built-in socket library functions. The communication between the client and the simulator server uses command and data messages. For example, when the simulator core is ready to run after initialization, it sends a message "started" to the client. The client waits for this message and proceeds with next step. 


\subsection{Software Development Flow}

This section will start from the classical PCAM multicore programming methodology as a guide to developing software for ePUMA. The second part will introduce ePUMA software project management and the build system of ePUMA software using the compiler tools.

\subsubsection{PCAM Parallel Algorithm Design Methodology}

Parallel algorithm design for multicore platform needs the programmer to identify task parallelism in an application, which is usually done manually by the software programmer. The mapping of the partitioned tasks to hardware cores and the scheduling are design challenges for multicore programming. PCAM [71] is an acronym of four design stages to guide the design of parallel applications. The steps are listed as follows:

1. Partitioning: The first step is to decompose the computation problem into small tasks. The purpose is to recognize the parallelism, without considering the hardware capability.

2. Communication: This step defines the communication between tasks, including data dependency and communication load.

3. Agglomeration: This step combines small tasks into task groups to reduce communication load between task groups.

4. Mapping: The last step is to assign task groups to processors. The goal is to balance the computing load on processors, and minimize inter-processor communication.

To apply this general PCAM methodology to ePUMA, the programmer needs to consider the special features of ePUMA hardware. For example, the communication can be overlapped by using the triple LVM buffers, and the data broadcasting of the stream network can save interprocessor communication cost. The programmer also needs to understand the computing characteristics of the tasks when mapping them to 


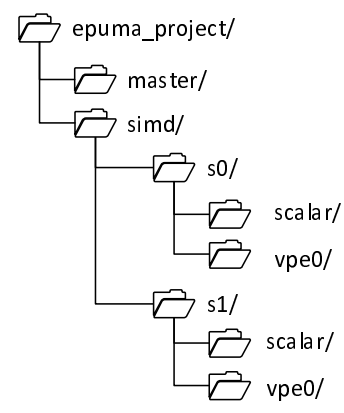

Figure 7.1: ePUMA software project folder structure

the processor cores, since we have both scalar core and vector engines in ePUMA. The scalar core is suitable for control rich tasks and sequential data processing such as bitstream parsing. The vector engine is suitable for data parallel execution such as matrix computing. For data flow based applications, a practical method is to divide tasks based on the computing stages in the signal processing flow, and balance the computing load on processor cores by grouping small tasks on one processor or decomposing a data-intensive task into parallel tasks on multiple processors.

\subsection{2 ePUMA Software Project}

ePUMA software project contains source codes for different processor cores, which require different compiler or assembler tools. The master and the SIMD scalar core use C compilers. The SIMD VPE core uses the SIMD assembler. The source code needs to be processed in a bottom-up order and linked by the linker tool to constitute the final software image.

Figure 7.1 shows a project folder structure as a template. In an ePUMA software project, there are three types of subfolders that contain source codes: master, scalar and vpe. The master folder contains the main program for the master core. There is only one master folder for each application. The scalar folder contains the software program for SIMD scalar core. A project usually has multiple scalar programs to execute on 
different SIMD cores. The vpe folder contains the SIMD program to be loaded to VPM at one time. It can include one or multiple tasks. Each task can be viewed as a standalone function. The tasks are combined statically into one VPE program. The VPE assembler generates a header file which contains pointer values to each task. The scalar program can include this header file and issue different VPE tasks by using the task pointers. Before the master program is compiled, the SIMD kernel programs including the scalar programs and the VPE programs need to be linked together, so that the master can use the reference of kernel locations in its DMA function to load kernel from main memory to on-chip program memory.

According to the dependency of task pointers and kernel references, the building of ePUMA software project should follow a bottom-up process in the following 5 steps:

1. Use the assembler to assemble each VPE program in the vpe folders, and generate vpe header file for task pointers.

2. Use the scalar compiler to compile SIMD scalar program in the scalar folders.

3. Use the linker tool to pack all SIMD coprocessor's scalar and vpe programs into one kernel binary image, and generate kernel header file.

4. Use the $C$ compiler to compile the master program in the master folder.

5. Append the master binary at the end of the kernel package to form the final software image.

\subsection{Multitasking Framework}

The ePUMA hardware platform consists of 8 SIMD coprocessors to offer high computing power. The execution of each SIMD core relies on the 
master to load program, provide input data and collect the computing result. If less than 8 SIMD processors are required for one DSP application, the rest of the SIMD cores can be shut down to save power. However, we can fully utilize the computing power of all cores by enabling multitasking on the master core. It is possible because the computing load of the master core is much lower when computing kernels are moved to SIMD cores. In addition, since ePUMA targets a wide range of applications, including communication, multimedia and graphics computing, the DSP subsystem needs to respond to various computing requests from the host system. The requests can be regular signal processing tasks at fixed time intervals (triggered by a timer) such as video decoding, or tasks triggered by external events such as analog sensors, or DSP computing tasks triggered by host CPU applications. The interrupt controller and timers are necessary hardware peripherals for the master core to support multitasking, which are included in ePUMA hardware architecture. The rest part of this section will introduce the basic multitasking framework for ePUMA.

\subsection{1 ePUMA Simple OS}

The multitasking operating system for ePUMA considers the predictable computing characteristics of the target applications and the memory architecture of the hardware platform. The Simple OS uses static memory management. The external memory should be managed by the host processor only. Dynamic memory allocation is done by the host controller before it sends a task to ePUMA. For example, frame buffers for a video decoder should be allocated in the external memory by the host controller based on the frame size. A pointer to the frame buffer is passed to the ePUMA decoder subsystem as an input parameter. The Simple OS is a preemptive OS. It uses interrupt events to switch between tasks. The interrupt controller of ePUMA master core supports up to 32 input sources. Three are used for timers, and one for host CPU interrupt. The 28 remaining interrupt pins are reserved for other hardware components 


\begin{tabular}{|c|c|c|c|}
\hline & PCB data & & PCB data \\
\hline 0 & state reg. & 9 & $\mathrm{r} 7$ \\
1 & pc+4 & 10 & $\mathrm{r} 8$ \\
2 & $\mathrm{r} 0$ & 11 & $\mathrm{r} 9$ \\
3 & $\mathrm{r} 1$ & 12 & $\mathrm{r} 10$ \\
4 & $\mathrm{r} 2$ & 13 & $\mathrm{r} 11$ \\
5 & $\mathrm{r} 3$ & 14 & $\mathrm{r} 12$ \\
6 & $\mathrm{r} 4$ & 15 & $\mathrm{r} 13$ \\
7 & $\mathrm{r} 5$ & 16 & $\mathrm{r} 14$ \\
8 & $\mathrm{r} 6$ & & \\
\hline
\end{tabular}

Table 7.2: PCB data structure

in the host SoC system such as analog to digital sensors. It is important to keep the OS size small to fit all code in the on-chip scratchpad memories for better performance. The code size of the current Simple OS framework without application code and the master library is less than $2 \mathrm{~KB}$ (1904 bytes).

In the Simple OS each task has its own stack. Task switching doesn't affect the software stacks. However, the registers of the master core needs to be saved during context switching. The register values are stored in a data structure called Process Control Block (PCB) in the memory, which is allocated at compile time for each static task in the OS kernel. The PCB data structure is listed in Table 7.2. Each PCB task block corresponds to one static task of the OS and uses 68 bytes memory space to keep a copy of 16 registers and 1 stack pointer. All the PCB task blocks need to be initialized during the OS booting time.

The memory management of the OS kernel is shown in the Figure 7.2. It uses the on-chip scratch pad memory as the primary memory. All OS kernel code is kept in the on-chip memory. For large application tasks, non-critical application code is moved to external memory and accessed through cache.

The interrupt handler of the Simple OS doesn't support nested in- 


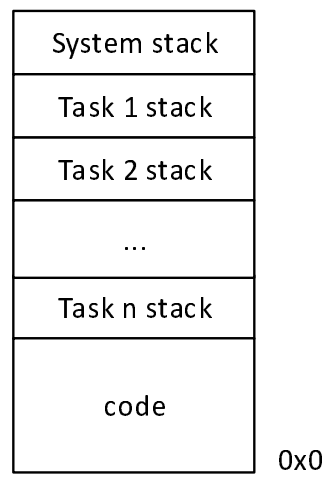

Figure 7.2: Simple OS memory mapping

terrupts. New interrupts are stalled when the master core is serving an accepted interrupt. Advanced interrupt handler functions can be used to handle prioritized interrupt requests.

The scheduler function in the OS kernel switches tasks using the PCB table. This function is called in the interrupt handling routine. The context switch will save the current processor states into the current task's PCB block and load the next task's context values from the PCB table. The Simple OS contains only static tasks, which can not be created or deleted at runtime. However, a task can be enabled or disabled for execution. Inter-task communication is rare on the ePUMA platform because the static tasks run independently of each other.

Since all OS tasks share the single DMA controller to transfer data to/from the SIMD coprocessors, conflicts for the use of the DMA controller may happen. One conflict is in the DMA configuration. The application task can be destroyed if the DMA task configuration in the current OS task is overwritten by another process. One solution is to divide the DMA configuration buffers and assign them to different OS tasks. Another conflict might happen in the DMA control process. One example case is the context switching takes place just before the current task is going to start the DMA engine. Then the DMA controller is started in the new process for a different data transfer. When the OS switches back to 
the previous task, while the DMA is serving another SIMD coprocessor, it will break the program flow. To solve this problem we use a device driver to manage the DMA controller. Using the device driver, application tasks cannot access the DMA controller directly. It needs to gain control of the shared device from the OS kernel. The device driver uses a global semaphore to prevent other tasks from accessing the same device. When the device driver function is running, the master core is in supervision mode, and all interrupts are disabled. The interrupt will reenabled when the master core returns from the driver function and change back to the user mode.

\subsection{Summary}

This chapter introduces ePUMA software development flow, tools support and software project management. The software development uses a kernel based parallel programming model. The application program contains separate code for the master core, SIMD scalar core, and SIMD VPE core, which are compiled by different ePUMA tools. Examples of the master program, kernel scalar control code and kernel SIMD code are used to demonstrate the software programming of ePUMA. The PCAM parallel algorithm design methodology is introduced as a general flow of multicore programming. At the end, a multitasking framework designed for ePUMA is presented to support concurrent task execution on the multicore platform. 


\section{Part III}

\section{Results and Conclusions}





\section{Chapter 8 \\ Application Implementations}

The contribution of application analysis and implementation is described in this chapter.

This chapter collects examples of application implementations on ePUMA in [65] [22] [23] [52]. It focuses on the data access and stream data communication instead of the kernel SIMD computing. The first two simple examples (syrk and matrix multiplication) demonstrate the usage of the unique features of ePUMA memory system. The convolutional decoding and LTE downlink signal processing are advanced examples to show the flexibility of ePUMA memory architecture in solving more complex problems in real applications.

\subsection{The BLAS syrk Routine}

The Basic Linear Algebra Subprograms (BLAS) standard [72] [73] defines a library of software interface functions to perform basic linear algebra operations such as vector and matrix multiplication. BLAS is a fundamental component of the core math functions, it is widely used in high performance computing. Most of today's hardware vendors have devel- 


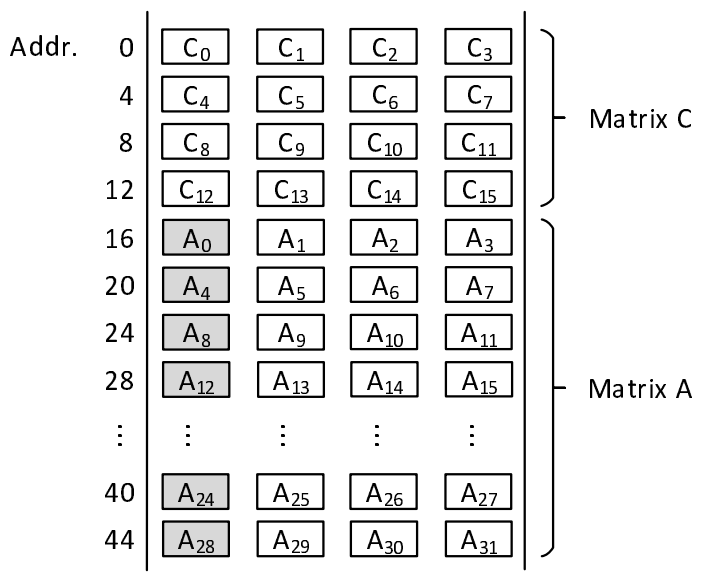

Figure 8.1: Input matrix data allocation in the main memory for the syrk example

oped optimized implementations of the BLAS operations, for example Intel Math Kernel Library [74], AMD Core Math Library [75], and Nvidia CUBLAS library [76].

We implement a BLAS level-3 routine syrk as an example to show the memory access flexibility offered by ePUMA memory subsystem. The syrk function performs a rank-k update of a symmetric matrix. Its operation, for the case trans $=T$, is defined in the equation below.

$$
C=\alpha * A^{T} * A+\beta * C, \text { for trans }=^{\prime} T^{\prime}
$$

Where $\mathrm{C}$ is an $\mathrm{n}$-by-n symmetric matrix, $\mathrm{A}$ is a k-by-n matrix, $\alpha$ and $\beta$ are scalar constants.

We choose a small matrix size of $\mathrm{n}=4$ and $\mathrm{k}=8$ for demonstration. In this example, we assume that a complete matrix $C$ is stored in the system main memory in a sequential order. Matrix $\mathrm{A}$ is also stored in a continuous space in the main memory, as shown in Figure 8.1. The selection and configuration of this example is for demonstration purpose to show the programming of ePUMA for the flexible parallel memory access.

One SIMD coprocessor is used to accelerate the syrk kernel comput- 


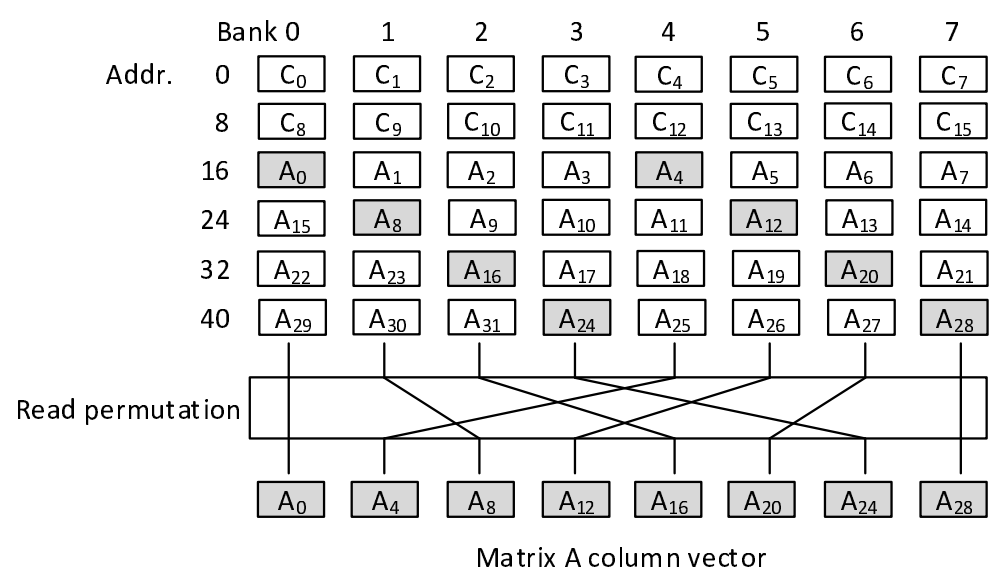

Figure 8.2: Matrix data allocation in SIMD LVM memory

ing. The host processor issues two DMA transactions to transfer input matrix A and C to the SIMD's local memory. The DMA controller has the same data width as the local multi-bank memory, eight 16-bit data can be transferred in one cycle. Transferring continuous data from the main memory through the memory controller and the system bus to the SIMD local memory can take advantage of the DRAM's paging property and the system interconnection's burst transfer feature to achieve a higher stream communication efficiency. Therefore the input stream from the external memory is transferred in sequential order.

The computing of $A^{T} * A$ requires column-wise access of matrix $\mathrm{A}$, as highlighted by the grey blocks in Figure 8.1. The SIMD coprocessor loads column vectors from $\mathrm{A}$ and then computes vector-vector multiplication with one SIMD instruction. The instruction performs multiplication and accumulation by one instruction. The same operation on different columns can be pipelined to achieve high computing throughput. The pipelined execution requires the access of vector data has minimum latency in order not to stall the pipeline. To access the parallel data without bank conflict, the column vector should be stored in different memory banks during DMA transaction. Matrix $\mathrm{C}$ has normal row-wise 
access.

The data allocation in SIMD local memory is illustrated in Figure 8.2. It can be seen that all the column vectors of matrix A are allocated in different banks. The SIMD core can access any column of matrix A without bank conflict.

The matrix data are shuffled in SIMD local memory for conflict free parallel access. The SIMD processor needs to be programmed to support the access of the re-ordered data using LUT based parallel memory addressing. The assembly code in Listing 8.1 illustrates the column vector access part in the syrk SIMD program.

Listing 8.1: SIMD program for the syrk example (load columns of matrix A without bank conflict)

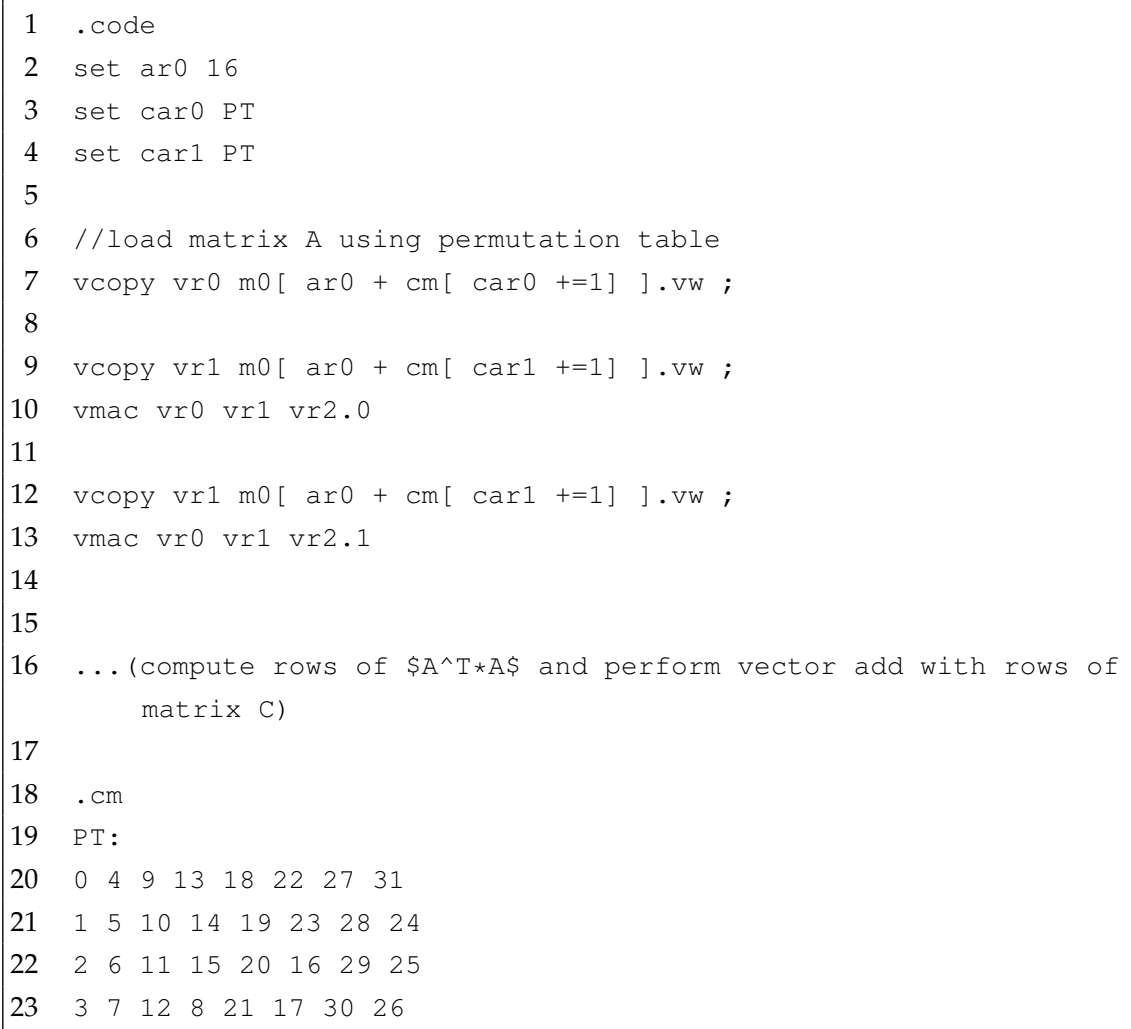

The vcopy instructions load the columns of matrix A to vector register 
vr0 and vr1, using a permutation table defined after the label PT in the constant data section (.cm). The SIMD computing is done by the vmac instruction for vector-vector multiplication and accumulation.

This example shows how the ePUMA SIMD local memory system supports software programmable data allocation. The SIMD data access overhead is minimized by allocating column vector data in different memory banks. For larger size and more complex kernel computing algorithms, software developers can take advantage of the parallel memory and optimize data allocations to reduce data access overhead and thus improve computing performance.

\section{2 $64 \times 64$ Matrix Multiplication}

Matrix-matrix multiplication is a common computing kernel of many signal processing algorithms. Complex value matrix multiplication is widely used in baseband processing in wireless communication and radar systems. Hence a fast matrix-matrix multiply brings benefit to these applications that require real-time processing performance. In this section we present a multicore parallel execution of matrix multiplication of two $64 \times 64$ complex matrices on ePUMA platform, using all the eight SIMD coprocessors. The design goal of ePUMA multicore architecture is to maximally reduce the control and communication overhead in predictable parallel processing. The main difficulty with this parallel matrix multiplication is the data access latency, in both bulk data movement from external memory to on-chip memory and parallel data access for data parallel computing. In this part we describe the parallel task distribution and scheduling, and evaluate the effectiveness of the ePUMA memory subsystem of reducing control and communication overhead.

\subsubsection{Task Distribution on Multiprocessors}

The $64 \times 64$ complex matrix multiplication is implemented on ePUMA platform using eight SIMD processors. The computing task is divided 


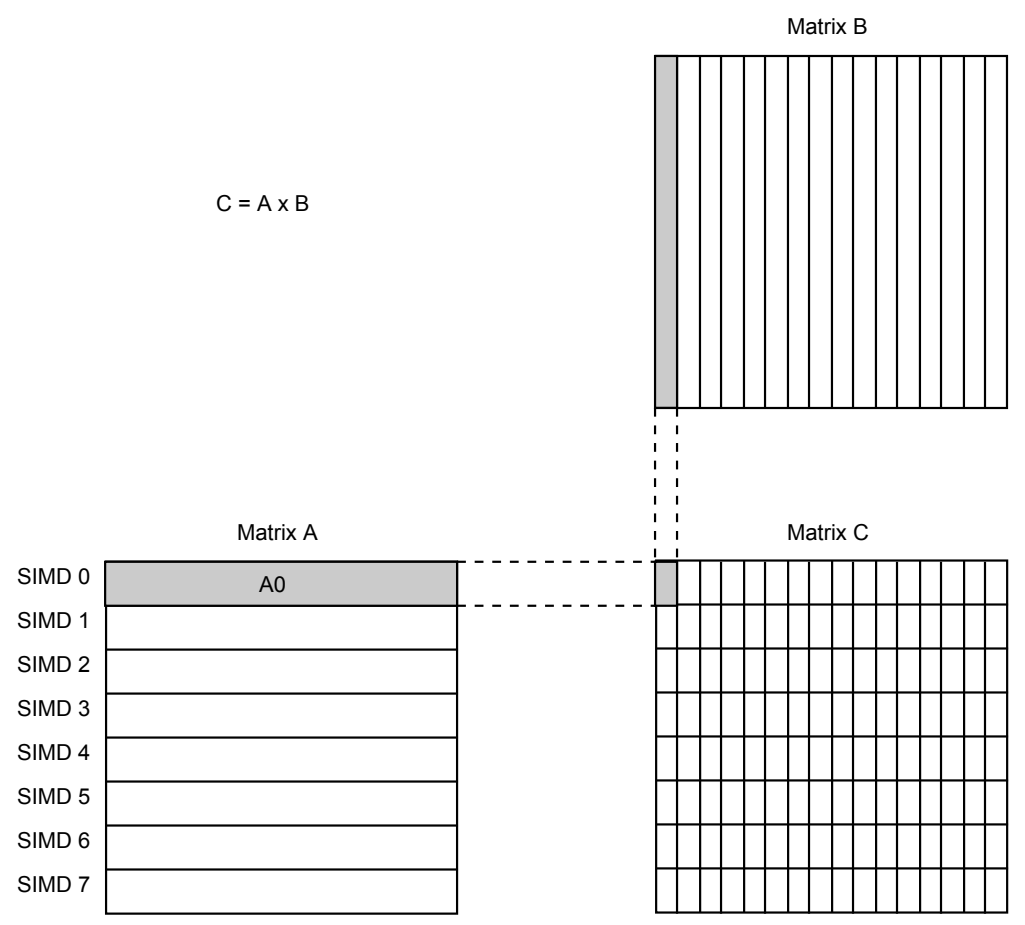

Figure 8.3: Task distribution of $64 \times 64$ complex matrix multiplication on eight SIMD processors

equally and assigned to eight SIMD processors, as shown in Figure 8.3. Each SIMD processor works on a subset of result matrix $C$, an $8 \times 64$ row block. The input data used by each SIMD processor for computing includes a row block of matrix A and the full matrix B. Since there is no data dependency between SIMD processors, they can run in parallel.

\subsubsection{Task Scheduling}

The master core executes the top level program for coprocessor configuration, program loading, data communication, and execution control. The pseudo-code in Listing 8.2 shows the task scheduling of parallel matrix multiplication on ePUMA multiprocessor. It consists of three parts: the prolog part, the kernel iterations, and the epilog part. Each SIMD ker- 
nel computes one $8 \times 4$ result block in C, using SIMD instruction for complex vector-vector multiplication and accumulation. Eight SIMD processors run in parallel in every iteration. 16 iterations are required to compute the result. It can be seen from the pseudo-code that the software also programs the DMA tasks for data communication. To minimize the memory access latency, we use DMA broadcasting to reduce redundant data transactions and ping-pong buffering to hide data communications behind kernel computing. The loop of DMA tasks in the prolog uses auto-initialization to save DMA task switching time.

Listing 8.2: $64 \times 64$ complex matrix multiplication on 8 SIMD processors

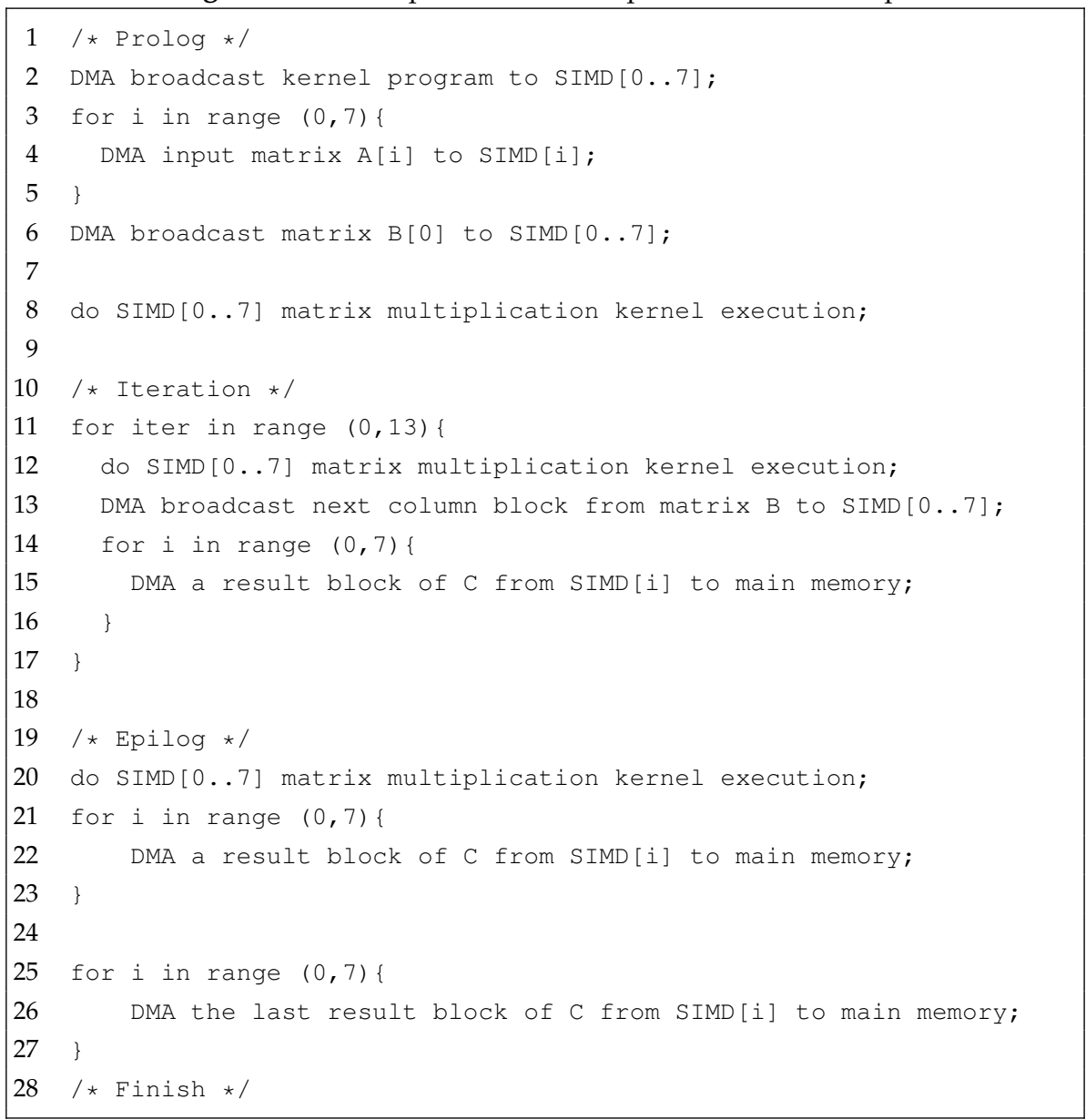




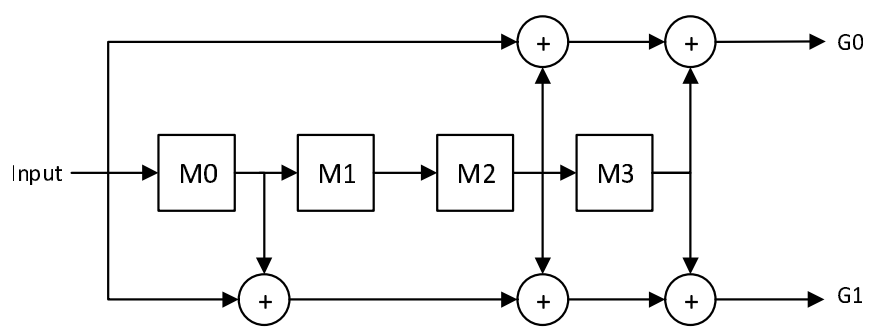

Figure 8.4: Convolutional encoder structure

\subsection{Convolutional Decoder}

Convolutional coding is an important error correction technique used in communication systems to overcome transmission distortions. A large variety of convolution codes are used in systems such as GSM, UMTS, IEEE802.16 and LTE (PDCCH channel). In this discussion, we consider the bit convolution code in modern communication systems. We will introduce the encoder structure, the Viterbi decoding algorithm, the puncturing operation and describe the implementation on ePUMA.

\subsubsection{Encoder}

A convolutional encoder generates output symbols depending on past input data. An encoder structure is illustrated in Figure 8.4. It consists of a chain of unit delays, and XOR function units which take inputs from different taps of the unit delays and calculate the output symbol. The combination of taps to generate the output symbol is described by a polynomial, which is selected to maximize the probability of decoding the correct sequence. The number of input bits used for encoder is defined as the constraint length $\mathrm{K}$, which equals to the number of unit delays plus one. The number of output bits per symbol is determined by the coding rate $1 / \mathrm{n}$. For the example in Figure 4 , the encoder has a configuration $\mathrm{K}=5, \mathrm{n}=2$. 


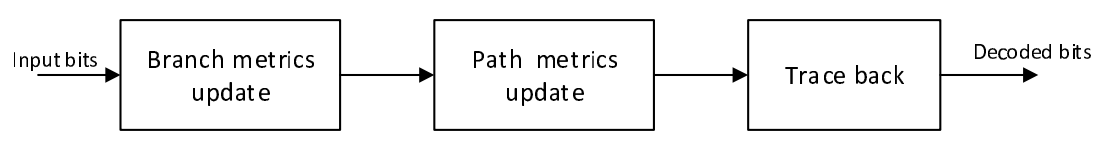

Figure 8.5: Viterbi decoder structure

\subsubsection{Viterbi Algorithm}

The Viterbi algorithm is a classical convolutional decoding algorithm based on maximum likelihood sequence decoding [77]. It has been implemented in a variety of DSP processors for digital baseband signal processing [78][79][80]. The Viterbi algorithm can be described by a trellis, where a transition takes place from one state at step $\mathrm{k}$ to a new state at step $\mathrm{k}+1$. In each step there are $\mathrm{N}=2^{K-1}$ nodes, representing all the states of the K-1 delay units. The decoding process is to find the most likely sequence of state transitions for a received sequence of symbols. The branch metrics and path metrics are used in decoding. The branch metrics represent the likelihood an input symbol corresponds to a signal, and the path metrics are the accumulated metrics from the previous states. A Viterbi decoder structure consists of three functional blocks: Branch metrics update, Path metrics update, and Trace back, as shown in Figure 8.5 .

\section{Branch metrics update}

The Branch metrics update computes the local distance between the received data and the expected symbol. The Viterbi algorithm can use either hard-bit input or soft-bit input. For hard-bit input, the metrics are updated by Hamming distance. With soft-bit input, the metrics are updated by Euclidean distance. Soft-bit can achieve better performance than hard-bit, since soft-bit contains information on the reliability level of the received symbols. The computation of the squared Euclidean distance is shown in Equation 8.2, where $S D_{i}$ is input soft-bit, and $G_{i}$ is the symbol on branch $\mathrm{j}$. In the equation, $G_{i} \in\{-1,+1\}$, the calculation of 
branch metrics (BM) can be further simplified to Equation 8.3 [78].

$$
\begin{gathered}
\operatorname{Distance}(j)=\sum_{i=0}^{n-1}\left(S D_{i}-G_{i}(j)\right)^{2} \\
B M(j)=\sum_{i=0}^{n-1} S D_{i} * G_{i}(j)
\end{gathered}
$$

In modern communication systems, each convolutional code word contains from 500 to 4800 symbols. Data level parallel processing for branch metrics update is important to improve performance. In a SIMD processor, multiple branch metrics can be updated in parallel.

\section{Path metrics update}

The path metrics is updated by choosing the optimum path for each state from its incoming branches. For binary convolutional codes, each state has two incoming branches from two preceding states, as shown in Figure 8.6. The path metrics update includes adding the corresponding BM to the path metrics of the preceding states, comparing the two new metrics values, and select the optimum one as the most possible path. This is also known as the Add-Compare-Select(ACS) operation. The branch selection in the ACS operation needs to be stored in order to reconstruct the codeword in the trace back phase.

For a convolution code with constraint length $\mathrm{K}$, there are $2^{K-1}$ states that need to be updated at each step. The number $\mathrm{K}$ varies between 4 and 9 in different communication systems [81], which corresponds to states number from 8 to 256 . The ACS operation is done in butterfly pairs, that is two continuous states at step $\mathrm{k}$ are connected with two states at step $\mathrm{k}+1$ with a stride $2^{K-2}$. A SIMD processor can update multiple Viterbi butterflies in parallel. However, when the SIMD lane size is less than the number of states, extra operations is required to permute the output vector to get continuous path metrics for the next iteration. Conventional SIMD architecture performs data permutation on vector registers. Large size vector permutation needs more load/store and permutation oper- 


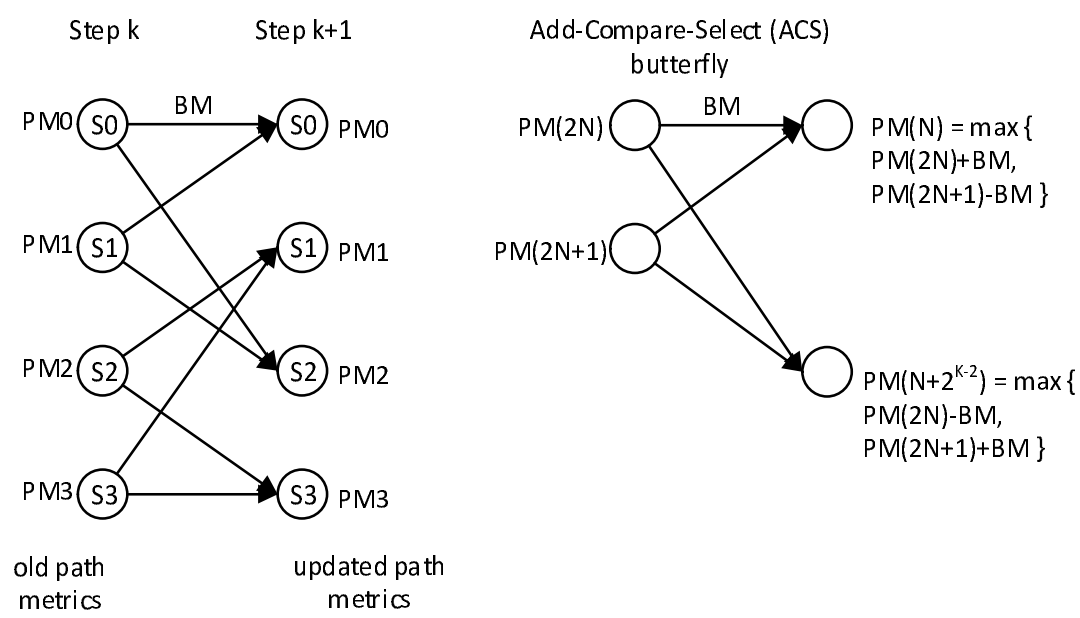

Figure 8.6: Viterbi decoder path metrics update

ations to reorganize data. ePUMA solves this problem by multi-bank memory and conflict free parallel access.

\section{Trace back}

The survival path in the path metrics update is stored to indicate the path to the previous state. The original data is decoded by tracing this maximum likelihood path backwards from the final state. Trace back is a scalar operation and requires much less MIPS compared to the ACS in path metrics update. It is not optimized in our SIMD implementation.

\section{Puncturing and de-puncturing}

Puncturing is used in communication systems such as UMTS, WiFi, and DVB-T to improve the coding rate. It is the processing stage after convolutional encoder which removes symbols in regular intervals from the encoded data. At the receiver side an inverse operation known as depuncturing is used, where each removed symbol is replaced by a null symbol. The null symbol has the minimum bias to the decoding process. 
In some de-puncturing operation [78], the dummy soft-bit 0000 and 1111 are inserted alternatively into the received soft decision bit stream as:

\begin{tabular}{|l|l|l|l|}
\hline$S D_{0}(0) S D_{1}(0)$ & $S D_{0}(1) 0000$ & $S D_{0}(2) S D_{1}(2)$ & $1111 S D_{1}(3)$ \\
\hline
\end{tabular}

Parallel de-puncturing on conventional SIMD architecture contains permutation overhead in shuffling data in vector registers. In ePUMA, this problem is solved by the parallel memory in a very efficient way with zero overhead.

\subsubsection{Implementation on ePUMA}

The following part will discuss how to implement the computing stages on ePUMA. The implementation of Viterbi computing kernels will use ePUMA SIMD instructions and the LUT based addressing of parallel memory. The goal is to achieve high ALU utilization and low data access overhead.

\section{Branch metrics update}

The computation in branch metrics update is shown in the following equations.

$$
\begin{aligned}
& B M_{0}=S D_{0}+S D_{1}+S D_{2} \\
& B M_{1}=S D_{0}+S D_{1}-S D_{2} \\
& B M_{2}=S D_{0}-S D_{1}+S D_{2} \\
& B M_{3}=S D_{0}-S D_{1}-S D_{2}
\end{aligned}
$$

For coding rate $1 / \mathrm{n}$, there are $2^{n}$ branches in each stage. Due to the symmetry of Viterbi butterfly structure, only $2^{n-1}$ branch metrics are required for each received symbol. Here we use the rate $1 / 3$ as an example. The four branch metrics for each received symbol are listed as below:

There are two solutions for the ePUMA implementation: using the VMAC instruction or the VADDSUB instruction. 
The VMAC solution uses the outer loop parallelization, that is, to compute the $S D_{0} \pm S D_{1}$ by the first VMAC instruction, and then compute $\pm S D_{2}$ by the second VMAC instruction. In the eight way data path, two symbols can be updated in parallel. Each $V M A C$ will execute eight multiplications and eight additions.

The scalar to vector conversion is required for the $V M A C$ operand. For example, when processing two symbols in parallel, the first four elements are copies of $S D_{0}(0)$ from the first symbol, the last four elements are $S D_{0}(1)$ from the second symbol. Some SIMD processor supports the scalar to vector conversion by the move instruction, for example the disjoint scalar to wide SIMD move in SODA [82]. ePUMA's parallel memory supports multiple read from the same address in the same memory bank (multiple read from different addresses in the same bank will cause conflict). In ePUMA, a scalar is expanded to a vector by the memory read directly. Therefore the scalar to vector conversion on ePUMA has zero overhead.

Another solution of branch metrics update is to use the VADDSUB instructions. The instructions are listed in Table 8.1. For VADDSUB2 and VADDSUB3, multiple symbols can be processed in parallel. These three

Table 8.1: VADDSUB instruction for branch metrics update

\begin{tabular}{|l|l|l|c|}
\hline Instruction & Input & Output & Parallel Symbols \\
\hline VADDSUB2 & $I_{0} I_{1}$ & $I_{0} \pm I_{1}$ & 4 \\
VADDSUB3 & $I_{0} I_{1} I_{2}$ & $I_{0} \pm I_{1} \pm I_{2}$ & 2 \\
VADDSUB4 & $I_{0} I_{1} I_{2} I_{3}$ & $I_{0} \pm I_{1} \pm I_{2} \pm I_{3}$ & 1 \\
\hline
\end{tabular}

VADDSUB instructions can be used for coding rate of $1 / 2,1 / 3$ and $1 / 4$ directly. For coding rate lower than $1 / 4$, combinations of these three instructions are used. For example in a $1 / 5$ convolution code, in each symbol interval we have five soft-bits $S D_{0} \sim S D_{4}$. We need to compute 16 branch metrics $B M_{0} \sim B M_{15}$. This can be done by combining one VADDSUB4 and two VADDSUB2 instructions, as below: 
VADDSUB4 $\left[S D_{0} . . S D_{3}\right]\left[B M_{0}^{\prime} . . B M_{7}^{\prime}\right]$

VADDSUB2 [ $\left.B M_{0}^{\prime} S D_{4} B M_{1}^{\prime} S D_{4} B M_{2}^{\prime} S D_{4} B M_{3}^{\prime} S D_{4}\right]\left[B M_{0} . . B M_{7}\right]$

VADDSUB2 [ $\left.B M_{4}^{\prime} S D_{4} B M_{5}^{\prime} S D_{4} B M_{6}^{\prime} S D_{4} B M_{7}^{\prime} S D_{4}\right]\left[B M_{8} . . B M_{15}\right]$

ePUMA SIMD processor operates on parallel memory directly, with no load store overhead. Also the replication of $S D_{4}$ in the VADDSUB2 instruction can be achieved by reading from the same memory cell, without scalar to vector conversion and permutation overhead. The VADDSUB instruction has higher ALU utilization. VADDSUB3 performs 12 add/sub operations in one instruction cycle, while VADDSUB4 performs 14 add/sub operations per cycle. Since multiplication with $\{+1,-1\}$ is replaced by addition in VADDSUB, power consumption on multipliers are saved.

\section{Path metrics update}

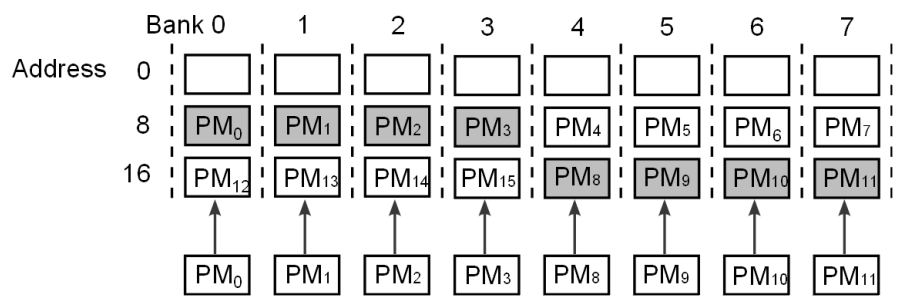

Figure 8.7: Path metric buffer data allocation of $K=5$ codes

The VACS instruction can perform 4 Viterbi butterfly operations in parallel. The path metrics are stored in the parallel memory. Two path metrics buffers are created to store the old and the new metrics. The old buffer and the new buffer are swapped after each symbol interval. The butterfly operation reads path metrics from consecutive locations. The updated path metrics are written back in two parts with a stride of $2^{K-2}$. The path metrics buffer in the parallel memory are organized for conflict-free read and write access. Figure 8.7 shows an example of path metrics data allocation for constraint length $\mathrm{K}=5$. The VACS reads [pm0..pm7] from 


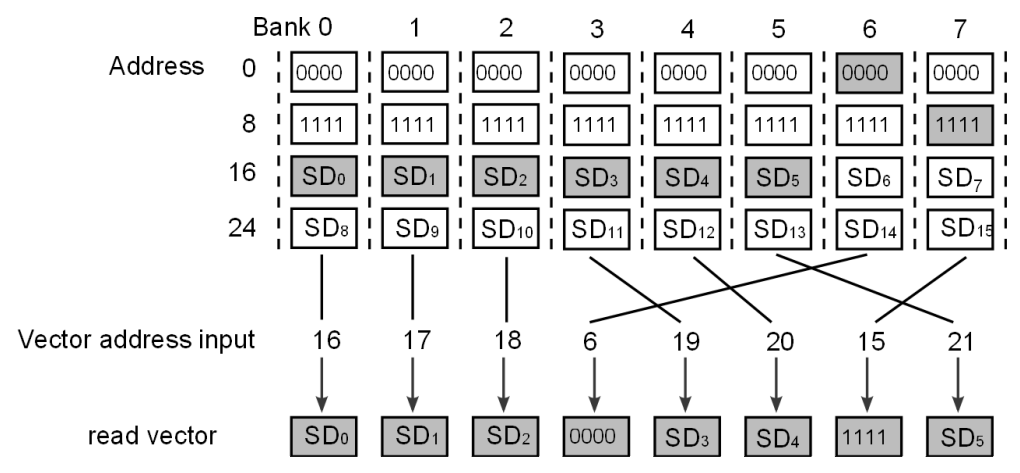

Figure 8.8: De-puncturing with ePUMA parallel memory

the old metrics buffer (four butterflies), and writes the updated value to [pm0..pm3,pm8..pm11] in the new metrics buffer. The data allocation shown in Figure 8.7 can support conflict free read and write access of the path metrics.

The survival paths is recorded in the flag register (for comparison operation) after the VACS instruction. It is moved to the vector memory at the end of each stage for the trace-back operation.

\section{Depuncturing}

The depuncturing is accelerated in ePUMA SIMD processor using the parallel memory. One constant vector in the parallel memory is initialized by copies of the null symbol. During depuncturing, the deleted softbit points to the free bank in the constant vector to load the null symbol.

The puncturing example shown in Section 8.3.2 requires the depuncturing process to alternatively insert ' 0000 ' and ' 1111 ' in the received symbols. Figure 8.8 shows the implementation of this depuncturing in ePUMA. We first initialize two constant vectors with ' 0000 's and '1111's. Then use vector copy instruction VCOPY to move data from input buffer with null symbols inserted to the depunctured buffer in another vector memory. 


\begin{tabular}{|c|c|c|c|c|c|}
\hline Rate & $\begin{array}{c}\text { ePUMA } \\
\text { VADDSUB }\end{array}$ & $\begin{array}{c}\text { ePUMA } \\
\text { VMAC }\end{array}$ & $\begin{array}{c}\text { TI C54x } \\
{[78]}\end{array}$ & $\begin{array}{c}\text { CEVA } \\
{[79]}\end{array}$ & $\begin{array}{c}\text { AltiVec } \\
{[80]}\end{array}$ \\
\hline $1 / 2$ & 0.25 & 0.25 & 5 & 4 & 0.75 \\
$1 / 3$ & 0.5 & 1 & 13 & 8 & 1.625 \\
$1 / 4$ & 1 & 3 & 33 & 16 & 3.25 \\
$1 / 6$ & 5 & 9 & 193 & 64 & 12.5 \\
$1 / 8$ & 25 & 33 & 1025 & 256 & 48.75 \\
\hline
\end{tabular}

Table 8.2: Cycles per symbol for branch metrics update

\subsubsection{Performance Comparison}

The performance evaluation results of the computing kernels of Viterbi algorithm on ePUMA are compared with other architectures, including TI C54x (two additions in parallel) [78], CEVA TeakLike III (four additions in parallel) [79], and PowerPC AltiVec (8-way SIMD) [80]. All these architectures have application specific instructions which can accelerate convolutional decoding. For example, TI uses the DADST/DSADT instruction for dual add/subtract operation, and the CMPS instruction for compare-select-store. CEVA has the ADD4W and ADDSUB2W instructions for parallel addition and subtraction of four words in one cycle. The AltiVec has the same SIMD width as ePUMA, it uses vector arithmetic instructions (vec_add and vec_min), and vector permutation instructions (vec_perm and vec_pack) to process 8 samples in parallel.

Table 8.2 lists the cycle costs per symbol for branch metrics update on different processors. It covers coding rates from $1 / 2$ to $1 / 8$, which are used by most convolutional codes in today's communication systems. The ePUMA VADDSUB solution achieves the high performance due to its deep execution pipeline with more ALU components, up to 14 adders are used in one instruction. The TI implementation uses the basic $A D-$ $D / S U B$ instruction. In CEVA, two additions are computed by one instruction. The AltiVec performs eight additions in parallel. The higher data path parallelism leads to a higher performance. The overhead on the three reference architectures is high because of the load/store and 


\begin{tabular}{|c|c|c|c|}
\hline ePUMA & TI C54x [78] & CEVA [79] & AltiVec[80] \\
\hline 0.25 & 5 & 2 & 3.5 \\
\hline
\end{tabular}

Table 8.3: Cycles per butterfly for path metrics update

permutation operations, which affect the performance. For example, in the CEVA implementation of coding rate $1 / 3,50 \%$ of the instructions are $s t 2 w$ for storing the result from the accumulator register to memory. ePUMA uses flexible multi-bank memory and two memory operands, the computing overhead is reduced to a large extent.

The computing capabilities for Viterbi ACS butterfly operation on different architectures are listed in Table 8.3. Each ACS butterfly requires four additions and two compare-and-select operations. Both TI and CEVA have special instructions for the ACS operation as ePUMA. The difference is in the level of parallelism. In TI C54x, two additions can be performed by one instruction, and one compare-select operation can be done by one instruction. Only one operand in the TI instruction can be a memory operand, extra load instructions are required to load another operand to register for computing. The CEVA processor can do four additions in one instruction, and spend another instruction for the two compare-and-select operations. Furthermore, the CEVA instruction can use two memory operands. The AltiVec implementation gets low performance out of its wider data path, because the AltiVec does not have a specific instruction for ACS operation. Addition, comparison and selection are performed by separate instructions. Even more, the same comparison is done twice, the first one compares two vectors by vec_min to assign the new path metrics, and the second comparison by vec_cmpgt to get the compare flag which indicates the survival path for trace back operation. ePUMA combines all the addition, comparison, and selection operations into one VACS instruction to fully utilize the ALU components in its deep execution pipeline. It uses two memory operands and manage the computing buffers in the multi-bank memory for conflict free parallel access to reduce data access overhead. 


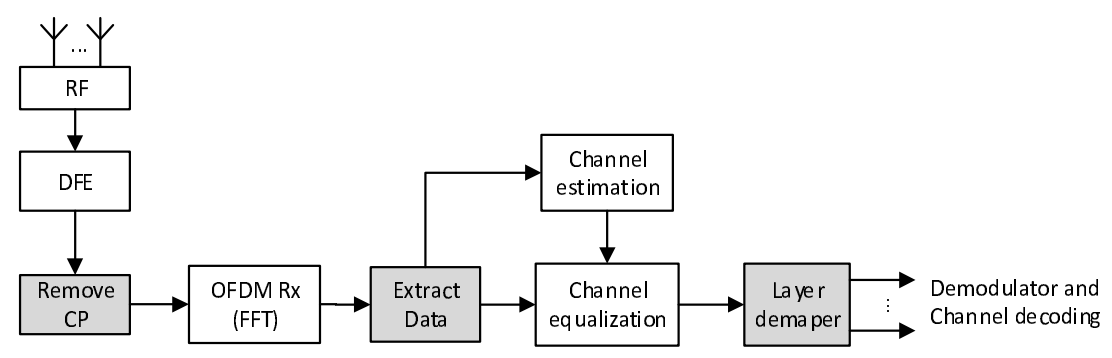

Figure 8.9: LTE downlink symbol processing data flow

\subsection{LTE Downlink Baseband Signal Processing}

The addressing complexity in advanced signal processing applications such as LTE baseband processing makes it hard to achieve high parallel computing efficiency due to the data access overhead. In this example we demonstrate the advantage of ePUMA platform for LTE downlink signal processing at the user end (UE) [83][84]. In the demonstration ePUMA is used only for symbol processing. Digital front end (DFE) and channel coding are not included. The system configuration uses $5 \mathrm{MHz}$ bandwidth and 2x2 MIMO. The DSP subsystem processes one sub-frame at each time, which contains 14 OFDM symbols (2 slots) from each receiving antenna.

\subsubsection{Signal Processing Flow and Computing Kernels}

Figure 8.9 shows the signal processing flow. After DFE, the data package contains both cyclic prefix (CP) and OFDM symbols. The first step is to remove the CP. The master controller will issue a DMA transfer to move the OFDM symbols from the main memory to SIMD's local parallel memory. The CPs are skipped by the DMA controller directly. The second step is the OFDM receiver, which consists of a group of FFT operations. In this example, this function block will process 28x 512-point FFT transforms. The size of FFT is determined by the LTE bandwidth, and it varies from $128(1.4 \mathrm{MHz})$ to $2048(20 \mathrm{MHz})$ [26]. The next step is to extract reference signals and data signals from the FFT output. The ref- 


\begin{tabular}{|l|l|r|}
\hline Function block & Computing kernel $^{1}$ & Computation load $^{2}$ \\
\hline OFDM Rx & 512-point FFT & $28 \times$ \\
\hline Channel & Complex multiplication & $800 \times$ \\
Estimation & Interpolation $^{3}$ & $3800 \times$ \\
\hline Channel & 2x2 matrix inversion & $3800 \times$ \\
Equalization & vector-matrix multiplication & \\
\hline
\end{tabular}

Table 8.4: Computing kernels in the reference model

Notes: 1 . For the LTE configuration of $5 \mathrm{MHz}$ bandwidth and 2x2 MIMO;

2 . The computation load is the number of kernel operations for processing 1 subframe (1ms); 3. The reference model [84] uses simple repeat function for interpolation. Since the scalar to vector expansion (repeat) in ePUMA has no extra cycle cost, the computation load is zero.

erence signals will be used for channel estimation. And the data signal is sent to the channel equalizer, which uses the estimated channel parameters to adjust the received data. Then the layer demapper will map the layer data to different codeword streams for further processing including demodulation, descrambler, and Turbo decoding.

In this signal processing flow, the function blocks for data manipulations (the grey boxes in Figure 8.9) can be realized by DMA communications at no processor computing cost. The rest of the function blocks are computing intensive tasks and will be assigned to different SIMD processors for parallel processing. Table 8.4 lists the computing kernels and computation load of these function blocks. The algorithms are selected according to the reference model [84]. The MIMO receiver uses direct $2 \times 2$ matrix inversion. The reference model chooses the simple repeat function to do interpolation in channel estimation, the computing complexity/load will increase if advanced interpolation algorithm is applied.

To demonstrate the implementation in ePUMA, we will use two examples to show the addressing complexities in this application. Since all computing data are complex numbers, and one complex number takes 
(1)

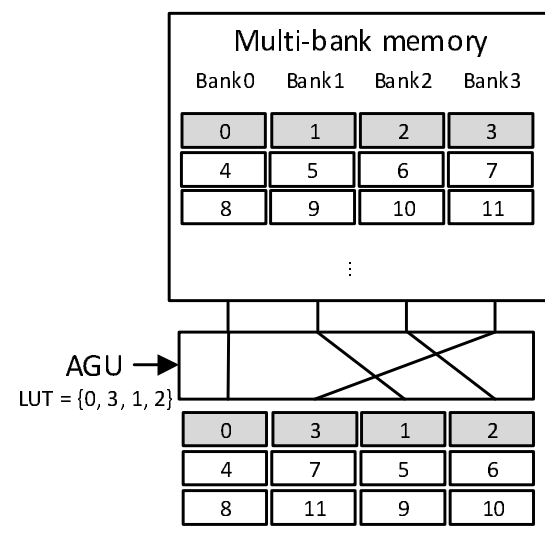

SIMD

Instruction CMULSUB $\left\{a_{i}, d_{i}, b_{i}, c_{i}\right\}$ (psuedo code)
(2)

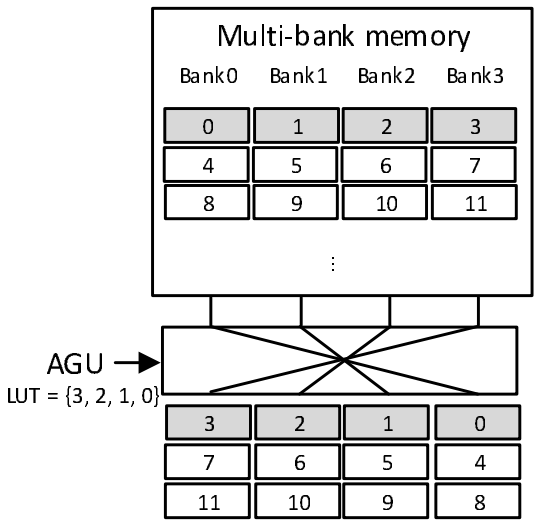

$\operatorname{CMUL}\left\{\mathrm{x}_{2 \mathrm{i}},-\mathrm{x}_{2 \mathrm{i}},-\mathrm{x}_{2 \mathrm{i}}, \mathrm{x}_{2 \mathrm{i}}\right\},\left\{\mathrm{d}_{\mathrm{i}}, \mathrm{c}_{\mathrm{i}}, \mathrm{b}_{\mathrm{i}}, \mathrm{a}_{\mathrm{i}}\right\}$ $i=0,1,2, \ldots, 3799$

Figure 8.10: Data permutation for SIMD computing

two 16-bit words in the multibank memory, for better illustration we use four bank memory (each memory uses 32-bit word) in SIMD local parallel memory in the following discussion.

\subsubsection{Addressing Complexity in SIMD Computing}

The addressing complexity of SIMD computing is demonstrated by the channel equalization kernel. The first operation in channel equalization is $2 \times 2$ matrix inversion, as illustrated in Equation 8.4.

$$
\left[\begin{array}{ll}
a & b \\
c & d
\end{array}\right]^{-1}=\frac{1}{a d-b c}\left[\begin{array}{cc}
d & -b \\
-c & a
\end{array}\right]
$$

The input data $\{a, b, c, d\}$ are received from DMA and stored in the local parallel memory in a consecutive memory space, as shown in Figure 8.10. There are 3800 such $2 \times 2$ matrices in one data packet. The computing is divided into the following three steps, and each step performs the same operation on 3800 input data sets. 
- Step 1: $x_{1}=a d-b c$

- Step 2: $x_{2}=1 / x_{1}$

- Step 3: $x_{3}=x_{2} \cdot *\left[\begin{array}{cc}d & -b \\ -c & a\end{array}\right]$

Step 1 uses the SIMD instruction CMULSUB which takes four complex numbers $\{x, y, z, w\}$ as input and compute the output $x^{*} y-z^{*} w$. Since the input are stored in the parallel memory in the order of $\{a, b, c, d\}$, we need to shuffle it to the SIMD computing order $\{a, d, b, c\}$ before performing the operation. Traditional SIMD architecture loads data into a vector register and uses data shuffle instructions to reorder the vector elements. Extra overhead on load/store and data shuffling are required. In ePUMA, this can be done by configuring the lookup table in the AGU to perform data permutation. Step 2 is a scalar operation, the SIMD processor can compute on four input data in parallel. Only regular vector access of four neighboring elements is required. For the dot product operation at Step 3, we would like to write the output in the order $\left\{x_{2} * d, x_{2} *(-c), x_{2} *(-b), x_{2} * a\right\}$, to ease the data access in the following step of vector-matrix multiplications, which uses the SIMD instruction to perform two vector-vector multiplication in parallel, so we shuffle the input to the order $\{d, c, b, a\}$, and perform the dot product. Another operation at this step is the scalar to vector copy of value $x_{2}$. This will be achieved by configuring the AGU to generate four same addresses, all point to $x_{2}$ in the memory. Data are duplicated at the output of the permutation network.

As we can see from the above analysis, step 1 and step 3 requires parallel memory access of the same input $\{a, b, c, d\}$ but in different orders. We configure the AGU to provide vector addresses such that the data are permuted when read from the multibank memory, as illustrated in Figure 8.10. This data permutation requires no extra cost in SIMD computing, thus the SIMD core can achieve high computing throughput. In this example, at step 1, the AGU is configured to generate an address sequence: 


$$
\{\{0,3,1,2\},\{4,7,5,6\},\{8,11,9,10\}, \ldots\}
$$

The AGU uses the vector offset $\{0,3,1,2\}$ in the lookup table, and adds it to the scalar base address which is post-incremented by 4 after every memory read.

At step 3, the AGU is configured to generate another address sequence: $\quad\{\{3,2,1,0\},\{7,6,5,4\},\{11,10,9,8\}, \ldots\}$

We choose another vector offset $\{3,2,1,0\}$ in the lookup table for data permutation.

The lookup table for address generation is pre-configured by the SIMD scalar controller software. The software programmers design the permutation patterns. These patterns are compiled and loaded into the lookup table of the AGU.

\subsubsection{Addressing Complexity in Stream Communication}

In a multicore platform, inter processor communication latency becomes critical to the overall system performance. Increasing network bandwidth (e.g. using wide data bus) is effective in improving streaming signal processing performance on multiprocessor. However, traditional DMA controller lack the flexible data access patterns for parallel memory. They either access local memory sequentially and then pack data to transfer, or transfer redundant data in the data packet. The communication efficiency is low in such approaches.

In this example, we use the reference signal extraction in LTE downlink signal processing to show the addressing complexity in stream communication. We also present the solution on ePUMA.

In the physical downlink channel of a LTE system, the receiver uses reference signals to estimate the radio channel and adjusts other received data to compensate for the channel effects. The reference signals are inserted during subcarrier mapping in both time and frequency domains. The locations of these reference signals are defined in the standard [26] and are determined by the bandwidth configuration and antenna port number. To avoid the high complexity of MIMO channel estimation at 


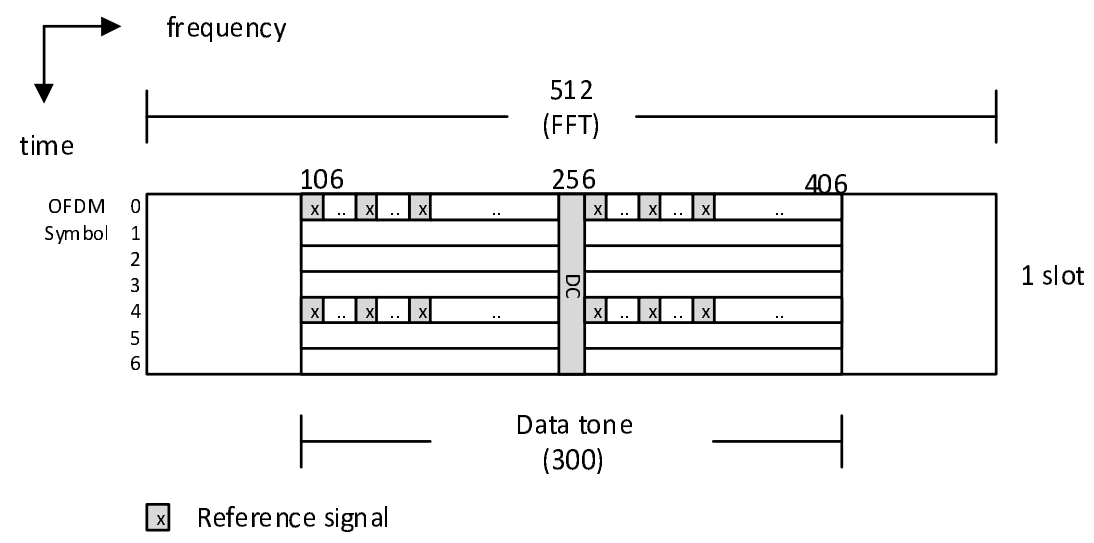

Figure 8.11: LTE downlink reference signal allocation

the UE, LTE standard defines the reference signals transmitted from multiple antennas to be orthogonal to each other, by using so called "silent" subcarriers. When extracting reference signals from one antenna, both active and "silent" references should be extracted for channel estimation. Figure 8.11 shows the location of these signals in one LTE slot which contains 7 OFDM symbols. They exist only in the symbol of id 0 and 4. The reference signals are located in every third subcarrier in these two symboles. Further more, as can be seen from the figure, only the middle 300 subcarriers are useful data tones, separated by the DC value in the middle.

The OFDM symbols are processed by the SIMD processor (input FFT) and stored continuously in its local vector memory. The next step is to extract the reference and data signals, and send them to two other SIMD processors for channel estimation and channel equalization. The data extraction and transfer are done by the DMA controller.

The extraction of reference signals can easily achieve parallel access since the neighbouring four data are stored in different memory banks except for the subcarriers around the DC. As illustrated in subfigure (1) of Figure 8.12, the reference signals have a constant stride 3 before the first DC at the address 256 . The reference signal 253 and 257 reside in the 
(1) Reference signal

\begin{tabular}{|c|c|c|c|}
\hline \multicolumn{4}{|c|}{ Multi-bank memory } \\
\hline Banko & Bank1 & Bank2 & Bank3 \\
\hline 0 & 1 & 2 & 3 \\
\hline \multicolumn{4}{|c|}{$\vdots$} \\
\hline 104 & 105 & 106 & 107 \\
\hline 108 & 109 & 110 & 111 \\
\hline 112 & 113 & 114 & 115 \\
\hline \multicolumn{4}{|c|}{$\vdots$} \\
\hline 248 & 249 & 250 & 251 \\
\hline 252 & 253 & 254 & 255 \\
\hline$\overline{D \text { DC }}$ & 257 & 258 & 259 \\
\hline \multicolumn{4}{|c|}{$\vdots$} \\
\hline \multicolumn{4}{|c|}{ Data permutation } \\
\hline 106 & 109 & 112 & 115 \\
\hline 118 & 121 & 124 & 127 \\
\hline 130 & 133 & 136 & 139 \\
\hline \multicolumn{4}{|c|}{$\vdots$} \\
\hline 250 & 253 & $x$ & $x$ \\
\hline
\end{tabular}

(2) Data signal

\begin{tabular}{|c|c|c|c|c|}
\hline \multicolumn{4}{|c|}{ Multi-bank memory } & \\
\hline Bank0 & Bank1 & Bank2 & Bank3 & \\
\hline 0 & 1 & 2 & 3 & \\
\hline \multicolumn{4}{|c|}{$\vdots$} & \\
\hline 104 & 105 & 106 & 107 & \\
\hline 108 & 109 & 110 & 111 & \\
\hline 112 & 113 & 114 & 115 & \\
\hline \multicolumn{4}{|c|}{$\vdots$} & \\
\hline 248 & 249 & 250 & 251 & \\
\hline 252 & 253 & 254 & 255 & \\
\hline $\mathrm{DC}$ & 257 & 258 & 259 & \\
\hline \multicolumn{4}{|c|}{$\vdots$} & \\
\hline & & & & \\
\hline \multicolumn{4}{|c|}{ Data permutation } & \\
\hline 107 & $x$ & $x$ & $x$ & Prolog \\
\hline 108 & 110 & 111 & 113 & \multirow{4}{*}{$\begin{array}{l}\text { Main } \\
\text { body }\end{array}$} \\
\hline 114 & 116 & 117 & 119 & \\
\hline \multicolumn{4}{|c|}{$\vdots$} & \\
\hline 246 & 248 & 249 & 251 & \\
\hline 252 & 254 & 255 & $\mathrm{x}$ & Epilog \\
\hline
\end{tabular}

$\mathrm{X}$ Dummy data transfer

Figure 8.12: LTE downlink reference signal extraction

same memory bank, reading them in parallel will have bank conflict. We split the reference data of one LTE slot into four DMA tasks. Each task contains 50 reference data and takes 13 cycles to transfer on the stream network.

To extract the data signals and transfer them to the target SIMD processor for channel equalization, we use the approach illustrated in subfigure (2) of Figure 8.12. As illustrated in the figure, the DMA transfer consists of three parts, the prolog data packet, the main body, and the epilog data packet. The main body is the regular part which can achieve parallel memory access for the majority of data signals. The prolog and epilog contains dummy data in the data packet. 


\begin{tabular}{|c|c|c|c|}
\hline SIMD core & Computing cycles & Total cycles & Efficiency \\
\hline 0,1 & 9578 & 13902 & $68.9 \%$ \\
2 & 2300 & 2751 & $83.6 \%$ \\
3 & 28500 & 32351 & $88.1 \%$ \\
\hline
\end{tabular}

Table 8.5: SIMD coprocessor computing efficiency

Notes: The cycle count is for processing 1 subframe in LTE downlink with a configuration of $5 \mathrm{MHz}$ bandwidth and 2x2 MIMO.

The DMA engine reads data in parallel and transfers them through the wide data bus to the target SIMD processor. At the receiver side, the AGU is configured to take out the dummy cells and write useful data in a continuous memory space.

\subsubsection{Performance Evaluation}

In the evaluation, we focus on two performance metrics, the SIMD computing efficiency and the DMA communication overhead. The evaluation is done on a cycle true and pipeline accurate simulator of ePUMA parallel DSP. In the example LTE configuration ( $5 \mathrm{MHz}$ bandwidth, $2 \times 2$ MIMO), four SIMD processors in ePUMA are used to process in parallel. SIMD 0 and 1 are used to compute the input FFT on data from two receiving antennas. SIMD 2 is used for channel estimation. And SIMD 3 is used for channel equalization.

\section{SIMD Computing Efficiency}

Table 8.5 shows the kernel program computing efficiency of each SIMD processor. The second column of the table lists the computing cycles used for SIMD arithmetic computing, such as the Butterfly instruction for FFT. The total cycles in the third column is the overall execution time, which includes the computing cycles plus overheads such as subroutine calls, branches, and register load/store. All the four cores used in this demonstration achieve a high computing efficiency (68\%-88\%). Tradi- 


\begin{tabular}{|c|c|c|c|}
\hline & DMA cycles* & Total cycles & Comm. overhead \\
\hline Sequential DMA & 23817 & 79989 & $29.8 \%$ \\
Parallel DMA & 5692 & 56488 & $10.1 \%$ \\
\hline
\end{tabular}

Table 8.6: DMA communication overhead

Note* : The DMA cycles count the communication time which is not overlapped with SIMD computing.

tional SIMD overhead on data shuffling and permutation are avoided in this architecture. We also found the major limitation of the computing efficiency in the current architecture is that we provide two parallel memories for each SIMD core, but for the operations that require three memory operands (two for inputs and one for output), we have to use the vector registers to keep one operand and exchange data with memory by load/store instructions. The computing overhead is increased by the register load/store. The FFT performed by SIMD 0 and 1 is a typical example of this operation. Thus the computing efficiency on these two cores are lower than others.

\section{DMA Communication Overhead}

Table 8.6 compares the system performance and DMA communication overhead of two solutions, one uses sequential DMA and another uses parallel DMA. The sequential DMA solution transfers a single data element per cycle through the network. It has low network implementation cost, and no memory conflicts on data access. But the communication latency is long, which results in low performance because of the high communication overhead. In the parallel DMA solution, multiple data elements are transferred in parallel through the network. The DMA can access local memory in parallel and transfer parallel data through the network. This improves the system performance and reduces the DMA communication overhead from $29.8 \%$ to $10.1 \%$. The network implementation cost of the parallel DMA solution should be considered. However, since ePUMA uses the message passing communication model and the 
streaming network is implemented as a none-addressable network, the implementation cost is much lower than traditional high bandwidth bus, as shown by the synthesis results in Chapter 9 .

\subsection{Other Applications on ePUMA}

In addition to the above implementations, we also collaborate with other research groups at Linköping University and Beijing Institute of Technology to develop applications and demonstrations on ePUMA in the area of computer graphics [85][86][87], multimedia applications [20], and general DSP kernels [88][89]. 


\section{Chapter 9 \\ Performance and Area \\ Evaluation}

This chapter uses simulation and synthesis results to evaluate the performance and area of the memory subsystem and the multicore system.

\subsection{SIMD Computing Efficiency}

The evaluation tests are executed on an ePUMA cycle-true and pipelineaccurate simulator. We have developed an architecture simulator including components of master controller, SIMD coprocessor, on-chip network and memory subsystem. The ePUMA SIMD computing efficiency is evaluated using the ratio $R$, where

$$
R=\frac{\text { Total execution cycles }}{\text { Arithmetic instructions }}
$$

Table 9.1 lists the evaluation results of selected DSP kernels. In the table, the theoretical limit shows the number of SIMD operations (8-way) required for the kernel computing. The SIMD processor is capable of executing one such operation per cycle, so this number is equal to the number of arithmetic instructions in Equation 9.1. The total cycle count lists the kernel execution time in simulator. In addition to the computing instructions, this cycle count also includes SIMD execution overheads 


\begin{tabular}{|l|c|c|c|}
\hline Algorithm & Theoretical limit & Total cycles & $\mathrm{R}$ \\
\hline $64 \times 64$ complex matrix mul. & 65536 & 66252 & 1.01 \\
$64 \times 64$ LU fact & 44972 & 58500 & 1.30 \\
$64 \times 64$ QR fact & 98944 & 115500 & 1.17 \\
1k radix-2 FFT & 1280 & 1710 & 1.34 \\
4 k radix-2 FFT & 6144 & 7635 & 1.24 \\
8 k 8-tap real FIR & 8192 & 8212 & 1.00 \\
$2 k$ 48-tap complex FIR & 24576 & 26679 & 1.09 \\
\hline
\end{tabular}

Table 9.1: DSP kernel evaluation results [1][65] C) 2010-2011 IEEE

such as data load/store, branch latency, and pipeline synchronization slots. The ratio $\mathrm{R}$ in the table shows that the SIMD processing overhead is about $10 \%$ to $30 \%$, which implies a high processing efficiency. In [22], we have evaluated the ratio $\mathrm{R}$ in a non-optimized 8-way SIMD processor architecture, where the multi-bank memory only supports sequential addressing as in most existing SIMD processors and the programmer has to use data permutation instructions to shuffle vector data for SIMD computing. The ratio $\mathrm{R}$ is 7.47 for $64 \times 64$ matrix multiplication in the non-optimized architecture because of the overhead on data shuffling.

There is potential overhead introduced by the ePUMA parallel memory architecture, that is, the loading of a permutation table as part of the kernel program consumes DMA time. The permutation table size varies in different applications. However, all the currently implemented benchmarking kernels show that the table size is usually small. The automatic permutation tool [60] can help to find the optimal permutation table with a minimum size.

\subsection{Memory Subsystem Implementation Cost}

The RTL design is synthesized using Synopsys Design Compiler with ST 65nm CMOS library. The clock frequency is set to $300 \mathrm{MHz}$. The logic sizes of the submodules are listed in Table 9.2. The SIMD parallel mem- 
ory shows high implementation cost as expected. This is because the permutation logic for the multi-bank memory requires a large number of multiplexers. This is the cost we have to pay for the flexibility of parallel memory access.

\begin{tabular}{|l|r|}
\hline Submodules & $\begin{array}{r}\text { Logic size } \\
\text { (kgates) }\end{array}$ \\
\hline Streaming Network & 17.2 \\
Master DMA & 7.9 \\
SIMD PPU & 8.5 \\
SIMD parallel memory & 24.1 \\
\hline
\end{tabular}

Table 9.2: Synthesize result (ST 65nm CMOS)

The streaming network is compared with two other Wishbone SoC buses [90] with the same 128-bit wide data bus, as shown in Table 9.3. It uses $30 \%$ logic gates of the Wishbone crossbar to provide the same communication bandwidth. The shared bus has the minimum implementation cost, but its low bandwidth makes it not suitable for streaming signal processing.

\begin{tabular}{|l|r|r|}
\hline Network & $\begin{array}{r}\text { Logic size } \\
\text { (128-bit data bus, } 8 \text { cores) }\end{array}$ & $\begin{array}{r}\text { Max. bandwidth } \\
\text { (bits/cycle) }\end{array}$ \\
\hline Stream network & 17.2 & 1024 \\
Wishbone shared bus & 6.4 & 128 \\
Wishbone crossbar & 56.6 & 1024 \\
\hline
\end{tabular}

Table 9.3: Network implementation cost (ST 65nm CMOS)

\subsection{System Implementation Cost}

The hardware implementation cost of ePUMA system is listed in Table 9.4. The table lists the hardware cost of the sub-components. The ePUMA 


\begin{tabular}{|l|l|r|r|r|r|}
\hline & Subblock & $\begin{array}{r}\text { Mem. } \\
\text { size }\end{array}$ & $\begin{array}{r}\text { Logic } \\
\text { gates }\end{array}$ & $\begin{array}{r}\text { Num. of } \\
\text { Inst. }\end{array}$ & $\begin{array}{r}\text { Area } \\
\left(\mathrm{mm}^{2}\right)\end{array}$ \\
\hline Master core & - & $256 \mathrm{~KB}$ & $70 \mathrm{k}^{*}$ & 1 & 2.2 \\
\hline SIMD core & Scalar & $32 \mathrm{~KB}$ & $50 \mathrm{k}^{*}$ & 8 & 2.8 \\
& VPE & $16 \mathrm{~KB}$ & $300 \mathrm{k}^{*}$ & 8 & 5.6 \\
& Local store & $192 \mathrm{~KB}$ & $32.6 \mathrm{k}$ & 8 & 12.5 \\
\hline Interconnection & Star network & - & $8 \mathrm{k}$ & 1 & 0.016 \\
& Ring network & - & $17.2 \mathrm{k}$ & 1 & 0.034 \\
\hline Total & & $2176 \mathrm{~KB}$ & $3156 \mathrm{k}$ & & 23 \\
\hline
\end{tabular}

Table 9.4: ePUMA implementation cost (* The implementation of the master controller and the SIMD processor core is ongoing, the gate count number is an estimation based on the current implementation.)

system is configured as 1 master core and 8 SIMD cores. The on-chip program memory of the master core is $256 \mathrm{~KB}$. The scalar program memory of one SIMD is $32 \mathrm{~KB}$, and the vector program size of one VPE engine is 16KB. Each SIMD coprocessor contains three LVM memories, each LVM is $64 \mathrm{~KB}$. Both the Star and the Ring networks choose a non-bufferable implementation, and the Ring network uses a high bandwidth 8-ring topology.

The total hardware cost is compared with the Cell multicore processor, the Imagine stream processor, and the Apple embedded GPU in the latest Apple A7 application processor. The Cell engine and the Imagine processor are complete chips with external memory controller, while ePUMA and Apple GPU are subsystems in a SoC. The ePUMA design in this synthesis uses a default configuration: each SIMD coprocessor contains one scalar and one VPE engine without ASIC accelerator. The total number of cores is $(1 \times$ Scalar $+1 \times \mathrm{XPE}) \times 8+1 \times$ Master $=17$. Cache for the master core is not included. Both ePUMA and Cell use SPM memories as local data buffers. The Imagine stream processor uses a stream register file as on-chip stream buffer, and the embedded GPU uses cache based memory architecture to execute data-dependent drawing functions. The original data are collected for different processes. They are scaled to $28 n-$ 


\begin{tabular}{|l|r|r|r|r|r|}
\hline & Cores & Mem. type & Process & $\begin{array}{r}\text { Area } \\
\left(\mathrm{mm}^{2}\right)\end{array}$ & $\begin{array}{r}\text { Approx. area } \\
\text { in 32nm }\left(\mathrm{mm}^{2}\right)\end{array}$ \\
\hline ePUMA & 17 & SPM & $65 \mathrm{~nm}$ & 23 & 6 \\
Cell [8] & 9 & Cache+SPM & $90 \mathrm{~nm}$ & 234 & 29 \\
Imagine [91] & 9 & SRF & $0.18 \mathrm{um}$ & 256 & 8 \\
A7 GPU* & 4 & Cache & $28 \mathrm{~nm}$ & 22 & 26 \\
\hline
\end{tabular}

Table 9.5: Comparison of multicore implementations (* The area of the A7 GPU is a non-official number. It assumes a $85 \%$ scaling from $32 \mathrm{~nm}$ to $28 \mathrm{~nm}$ )

$\mathrm{m}$ for comparison. The scaling calculation uses an estimation based on the assumption that the logic density is doubled between two main ITRS nodes, for example from $90 \mathrm{~nm}$ to $65 \mathrm{~nm}$, the silicon area shrinks to half.

From the table we can see that in the default configuration, the silicon area of ePUMA is less than $1 / 4$ of the complex multicore systems. The ePUMA in default configuration can be used as a general DSP subsystem to accelerate signal processing tasks such as matrix operations and FFT transforms. It can implement common programming interface such as OpenCL to accelerate host CPU applications by parallel computing. Modern GPUs increase the hardware utilization by using the programmable shader cores to do general purpose computing. The GPU hardware becomes much bigger and the size of cache increases in order to keep up with the increased number of processing cores. However, as we have analysed in the thesis, most DSP algorithms are predictable streaming computing and can not benefit from cache architecture as the general CPU architecture. A separate DSP subsystem with a different memory architecture optimized for predictable signal processing as a complementary component to the GPU can help to offload its computing load and reduce the number of cores and size of cache. In addition, an extended ePUMA subsystem can include ASIC datapaths attached to each SIMD coprocessor to take on more responsibilities in application specific computing for example video codec. 


\section{Chapter 10 \\ Conclusions and Future \\ Work}

As the computing complexity of embedded signal processing applications continues to grow, various Application Specific Integrated Circuits (ASIC) accelerators and Digital Signal Processor (DSP) subsystems are added to a System-on-Chip ( $\mathrm{SoC}$ ) solution to extend the computing capability. The growth in the number of SoC components adds load to the interconnection network, increases the silicon cost, and lowers the performance to power ratio. The additional control and communication overheads further reduce the computing efficiency of the parallel platform. There is a common architecture design trend in both general purpose computers and embedded processors to combine a host CPU with a programmable parallel processing unit such as GPU to accelerate general computing by a multicore parallel processor. In embedded SoC processors, the major DSP subsystems, including the communication modem, graphics engine, and video codec, are already accelerated by application specific programmable cores. This dissertation presents a novel parallel DSP subsystem (ePUMA) to target a wide range of embedded signal processing including communication, multimedia applications and more general purpose parallel computing.

The focus of this thesis is on designing a low overhead memory sub- 
system for the ePUMA parallel DSP. It starts from investigating the computing characteristics of streaming DSP applications and different parallel processor architectures. The memory subsystem is designed to meet the caching characteristics of streaming applications and to reduce the performance obstacles of the parallel processor.

The ePUMA processor is based on a master-slave heterogeneous multicore architecture. One master core performs the central control, and multiple SIMD coprocessors work in parallel to offer the majority of the computing power. The interconnection of the processing cores uses a combination of Star and Ring stream networks, and a serial bus for interprocessor notifications. The stream networks use non-address and unlimited burst transfer to move data between external memory and onchip memories. Parallel memory and a transitioned memory architecture are implemented as the on-chip local memory system for each SIMD coprocessor. The stream data is shuffled in the local multibank memory to provide conflict free parallel data access for SIMD computing. The addressing unit uses a lookup table to store the access patterns. The transitioned local memory architecture can overlap data communication with SIMD computing.

The implementation of signal processing applications on ePUMA shows a high computing efficiency and high ALU utilization of the hardware platform for signal processing applications with predictability in both data access patterns and task scheduling. The communication overhead is reduced because of the overlapped DMA and the high speed stream transfer on a wide data bus. The parallel memory architecture provides huge flexibility for accessing data in parallel. Both the stream communication and the parallel computing on the SIMD processor benefit from this.

The hardware cost of the stream network is low compared with conventional SoC interconnections. Both the Ring network physical layer and the Star network task buffer size are configurable. It uses less hardware to provide the same concurrent bandwidth as a conventional SoC bus. 
The future work of the memory subsystem design may include both hardware and software improvements. In the hardware design, clock gating and power gating on core level can be implemented to save power consumption. Customization of SIMD processors with different numbers of VPE engines and ASIC accelerators for specific applications can be evaluated. The interface to the system bus and the external memory can be further optimized to communicate streams through advanced buses or NoCs using long bursts and out-of-order transfer. Examples of such networks include the ARM AXI4 [51] and the Alteris NoC [92]. A network interface with an outstanding transaction manager and a re-order buffer is necessary between the central DMA controller and the system bus.

Future work in software can be the use of the multitasking framework to run two or more compute intensive applications, and implement more SIMD kernels to complete the software DSP library. A standard programming interface such as OpenCL can be ported to the ePUMA platform. 


\section{Bibliography}

[1] Dake Liu, A. Karlsson, J. Sohl, Jian Wang, M. Petersson, and Wenbiao Zhou. epuma embedded parallel dsp processor with unique memory access. In Information, Communications and Signal Processing (ICICS) 2011 8th International Conference on, pages 1-5, dec. 2011.

[2] David Seal. ARM architecture reference manual. Pearson Education, 2001.

[3] MIPS32 Architecture. Url http://www.imgtec.com/mips/mips32architecture.asp. Last access: 20th March, 2014.

[4] Texas Instruments. Tms320c64x technical overview. Texas Instruments, Feb, 2000.

[5] Anders Nilsson. Design of programmable multi-standard baseband processors. PhD thesis, Linköping University, 2007.

[6] Rizwan Asghar. Flexible Interleaving Subsystems for FEC in Baseband Processors. PhD thesis, Linköping University, Computer Engineering, The Institute of Technology, 2010.

[7] Khronos Open CL The open standard for parallel programming of heterogeneous systems. Url http://www.khronos.org/opencl/. Last access: 31st October, 2013.

[8] Michael Gschwind. The cell broadband engine: exploiting multiple levels of parallelism in a chip multiprocessor. International Journal of Parallel Programming, 35(3):233-262, 2007. 
[9] Jaejin Lee, Jungwon Kim, Sangmin Seo, Seungkyun Kim, Jungho Park, Honggyu Kim, Thanh Tuan Dao, Yongjin Cho, Sung Jong Seo, Seung Hak Lee, et al. An opencl framework for heterogeneous multicores with local memory. In Proceedings of the 19th international conference on Parallel architectures and compilation techniques, pages 193204. ACM, 2010.

[10] CUDA Nvidia. Programming guide, 2008.

[11] Khronos OpenCL Conformant Products. Url http://www.khronos.org/conformance/adopters/conformantproducts/. Last access: 31st October, 2013.

[12] TMS320C62xx User's Manual. Texas instruments inc. Dallas, TX, pages 18-23, 2002.

[13] Keith Diefendorff, Pradeep K Dubey, Ron Hochsprung, and HASH Scale. Altivec extension to powerpc accelerates media processing. Micro, IEEE, 20(2):85-95, 2000.

[14] CEVA Inc. A Low-Power DSP Architecture Framework for the Widest Array of Advanced Wireless Standards including LTE-Advanced, Wi-Fi 802.11ac, DVB-T2 and more.

[15] Deependra Talla, Lizy Kurian John, and Doug Burger. Bottlenecks in multimedia processing with simd style extensions and architectural enhancements. Computers, IEEE Transactions on, 52(8):10151031, 2003.

[16] Michael Gossel, Burghard Rebel, and Reiner Creutzburg. Memory Architecture and Parallel Access. Elsevier Science Inc., New York, NY, USA, 1994.

[17] Dake Liu. Embedded DSP Processor Design, Volume 2: Application Specific Instruction Set Processors, chapter 20. Morgan Kaufmann Publishers Inc., San Francisco, CA, USA, 2008. 
[18] David Tawei Wang. Modern dram memory systems: performance analysis and scheduling algorithm. 2005.

[19] David R Martinez, Robert A Bond, and M Michael Vai. High performance embedded computing handbook: A systems perspective. CRC Press, 2008.

[20] Yanjun Zhang, Wenbiao Zhou, Zhenyu Liu, Siye Wang, and Dake Liu. A high definition motion jpeg encoder based on epuma platform. Procedia Engineering, 29:2371-2375, 2012.

[21] Peng Xiaoyi. Benchmark of mpeg-2 video decoding on epuma multi-core dsp processor. Master's thesis, Linköping University, Computer Engineering, 2011.

[22] Joar Sohl, Jian Wang, and Dake Liu. Large matrix multiplication on a novel heterogeneous parallel dsp architecture. In Proceedings of the 8th International Symposium on Advanced Parallel Processing Technologies, APPT '09, pages 408-419, Berlin, Heidelberg, 2009. SpringerVerlag.

[23] Jian Wang, Andréas Karlsson, Joar Sohl, and Dake Liu. Convolutional Decoding on Deep-pipelined SIMD Processor with Flexible Parallel Memory. In Digital System Design (DSD), 2012, pages 529_ 532. IEEE, 2012.

[24] Di Wu, Johan Eilert, Dake Liu, Anders Nilsson, Eric Tell, and Erik Alfredsson. System architecture for 3gpp lte modem using a programmable baseband processor. In System-on-Chip, 2009. SOC 2009. International Symposium on, pages 132-137. IEEE, 2009.

[25] Orri Tomasson. Implementation of elementary functions for a fixed point simd dsp coprocessor. Master's thesis, Linköping University, December 2010.

[26] 3GPP Technical Specification Group Radio Access Network; Evolved Universal Terrestrial Radio Access (E-UTRA); Physical channels and 3GPP Modulation (Release 10), 3GPP TS 36.211 v10.0.0 
(2010-12). Url http://www.3gpp.org/ftp/specs/html-info/36series.htm. Last access: 22nd October, 2013.

[27] Di Wu, Rizwan Asghar, Yulin Huang, and Dake Liu. Implementation of a high-speed parallel turbo decoder for 3gpp lte terminals. In ASIC, 2009. ASICON'09. IEEE 8th International Conference on, pages 481-484. IEEE, 2009.

[28] ITUT Draft. Recommendation and final draft international standard of joint video specification (itu-t rec. h. 264 I iso/iec 14496-10 avc). Joint Video Team (JVT) of ISO/IEC MPEG and ITU-T VCEG, JVTG050, 2003.

[29] Jackie Neider, Tom Davis, and Mason Woo. OpenGL. Programming guide. Addison-Wesley, 1997.

[30] Michael C Shebanow. Pervasive massively multithreaded gpu processors. In Proceedings of the 6th ACM conference on Computing frontiers, pages 227-227. ACM, 2009.

[31] Richard M Russell. The cray-1 computer system. Communications of the ACM, 21(1):63-72, 1978.

[32] Mike Johnson. Superscalar microprocessor design. 1991.

[33] Joseph A Fisher. Very long instruction word architectures and the ELI512, volume 11. ACM, 1983.

[34] ARM NEON. Url http://www. arm. com/products/processors/technologies/-neon. php. Last access: 22nd October 2013, 2010.

[35] Xtensa Microprocessor Tensilica. Overview handbook.

[36] Texas Instruments. Tms320c6000 programmer's guide. Texas Instruments, 2000.

[37] Alex Peleg and Uri Weiser. Mmx technology extension to the intel architecture. Micro, IEEE, 16(4):42-50, 1996. 
[38] Nadeem Firasta, Mark Buxton, Paula Jinbo, Kaveh Nasri, and Shihjong Kuo. Intel avx: New frontiers in performance improvements and energy efficiency. Intel white paper, 2008.

[39] Jon Tyler, Jeff Lent, Anh Mather, and Huy Nguyen. Altivec tm: Bringing vector technology to the powerpc tm processor family. In Performance, Computing and Communications Conference, 1999 IEEE International, pages 437-444. IEEE, 1999.

[40] Dorit Naishlos, Marina Biberstein, Shay Ben-David, and Ayal Zaks. Vectorizing for a SIMdD DSP architecture. In CASES '03: Proceedings of the 2003 international conference on Compilers, architecture and synthesis for embedded systems, pages 2-11, New York, NY, USA, 2003. ACM.

[41] Hoseok Chang, Junho Cho, and Wonyong Sung. Compiler-based performance evaluation of an SIMD processor with a multi-bank memory unit. Signal Processing Systems, 56(2-3):249-260, 2009.

[42] Yuan Lin. Realizing Software Defined Radio - A Study in Designing Mobile Supercomputers. University of Michigan, 2008.

[43] SSE Intel. Programming reference. Intel's software network, sofwareprojects. intel. com/avx, 2:7, 2007.

[44] ARM Cortex. A8 technical reference manual. Rev. r2p1.

[45] David Bell and Greg Wood. Multicore programming guide. Application Report SPRAB27A. Texas Instruments, 2009.

[46] Stefania Sesia, Issam Toufik, and Matthew Baker. LTE: the UMTS long term evolution. Wiley Online Library, 2009.

[47] Sudeep Pasricha and Nikil Dutt. On-chip communication architectures: system on chip interconnect. Morgan Kaufmann, 2010.

[48] Michael Kistler, Michael Perrone, and Fabrizio Petrini. Cell multiprocessor communication network: Built for speed. IEEE micro, 26(3):10-23, 2006. 
[49] Praveen Raghavan, Satyakiran Munaga, Estela Rey Ramos, Andy Lambrechts, Murali Jayapala, Francky Catthoor, and Diederik Verkest. A customized cross-bar for data-shuffling in domain-specific simd processors. In Architecture of Computing Systems-ARCS 2007, pages 57-68. Springer, 2007.

[50] William J Dally and Brian Towles. Route packets, not wires: On-chip interconnection networks. In Design Automation Conference, 2001. Proceedings, pages 684-689. IEEE, 2001.

[51] AMBA. Amba axi and ace protocol specification. ARM, 2013.

[52] Jian Wang, Joar Sohl, and Dake Liu. Architectural support for reducing parallel processing overhead in an embedded multiprocessor. In Proceedings of the 2010 IEEE/IFIP International Conference on Embedded and Ubiquitous Computing, EUC '10, pages 47-52, Washington, DC, USA, 2010. IEEE Computer Society.

[53] RLDRAM DDR-DRAM. Micron technology.

[54] Yasuharu Sato, Takaaki Suzuki, Tadao Aikawa, SY Fujioka, Waichiro Fujieda, Hiroyuki Kobayashi, Hitoshi Ikeda, Takayuki Nagasawa, Akihiro Funyu, Y Fuji, et al. Fast cycle ram (fcram); a 20-ns random row access, pipe-lined operating dram. In VLSI Circuits, 1998. Digest of Technical Papers. 1998 Symposium on, pages 22-25. IEEE, 1998.

[55] David Tawei Wang. Modern Dram Memory Systems: Performance Analysis And Scheduling Algorithm. PhD thesis, University of Maryland, 2005.

[56] David A Patterson and John L Hennessy. Computer organization and design: the hardware/software interface. Newnes, 2013.

[57] Ujval J Kapasi, William J Dally, Scott Rixner, John D Owens, and Brucek Khailany. The imagine stream processor. In Computer Design: VLSI in Computers and Processors, 2002. Proceedings. 2002 IEEE International Conference on, pages 282-288. IEEE, 2002. 
[58] Peter Mattson. A programming system for the imagine media processor. PhD thesis, Stanford University, 2002.

[59] Bruce Jacob, Spencer Ng, and David Wang. Memory systems: cache, DRAM, disk. Morgan Kaufmann, 2010.

[60] Joar Sohl, Jian Wang, Andreas Karlsson, and Dake Liu. Automatic permutation for arbitrary static access patterns. In Parallel and Distributed Processing with Applications (ISPA), 2012 IEEE 10th International Symposium on, pages 215 -222, july 2012.

[61] Tzi-Dar Chiueh and Pei-Yun Tsai. OFDM baseband receiver design for wireless communications. John Wiley \& Sons, 2008.

[62] Jian Wang, Andreas Karlsson, Joar Sohl, Magnus Pettersson, and Dake Liu. A multi-level arbitration and topology free streaming network for chip multiprocessor. In ASIC (ASICON), 2011 IEEE 9th International Conference on, pages 153-158. IEEE, 2011.

[63] Khronos OpenGL ES The Standard for Embedded Accelerated 3D Graphics. Url http://www.khronos.org/opengles/. Last access: 31st October, 2013.

[64] Khronos Open MAX The Standard for Media Library Portability. Url http://www.khronos.org/openmax/. Last access: 31st October, 2013.

[65] Jian Wang, Joar Sohl, Olof Kraigher, and Liu Dake. Software programmable data allocation in multi-bank memory of simd processors. In Proceedings of the 2010 13th Euromicro Conference on Digital System Design: Architectures, Methods and Tools, pages 28-33. IEEE Computer Society, 2010.

[66] Hoseok Chang, Junho Cho, and Wonyong Sung. Compiler-based performance evaluation of an SIMD processor with a multi-bank memory unit. Signal Processing Systems, 56(2-3):249-260, 2009. 
[67] Chris Fallin, Xiangyao Yu, Gregory Nazario, and Onur Mutlu. A high-performance hierarchical ring on-chip interconnect with lowcost routers. Computer Architecture Lab, Carnegie Mellon Univ, Tech. Rep, 7:2011, 2011.

[68] DN Jayasimha, Bilal Zafar, and Yatin Hoskote. On-chip interconnection networks: why they are different and how to compare them. Platform Architecture Research, Intel Corporation, 2006.

[69] Jack Ganssle, Tammy Noergaard, Fred Eady, Lewin Edwards, David J Katz, Rick Gentile, Ken Arnold, Kamal Hyder, and Bob Perrin. Embedded Hardware: Know It All: Know It All, chapter 5.5.6, pages 231-233. Access Online via Elsevier, 2007.

[70] Dake Liu, Joar Sohl, and Jian Wang. Parallel computing and its architecture based on data access separated kernels. IJERTCS, International Journals Embedded and Real-Time Communication systems.

[71] Ian Foster. Designing and building parallel programs, volume 95. Addison-Wesley Reading, 1995.

[72] Blas (basic linear algebra subprograms). URL http://www.netlib.org/blas/, Last access: 22nd October 2013.

[73] J. J. Dongarra, Jeremy Du Croz, Sven Hammarling, and I. S. Duff. A set of level 3 basic linear algebra subprograms. ACM Trans. Math. Softw., 16(1):1-17, March 1990.

[74] MKL Intel. Intel math kernel library. URL http://software.intel.com/enus/intel-mkl, Last access: 22nd October 2013.

[75] ACML AMD. Core math library. URL http://developer.amd.com/acml.jsp, Last access: 22nd October 2013.

[76] CUDA Nvidia. Cublas library. URL https://developer.nvidia.com/cuBLAS, Last access: 22nd October 2013. 
[77] Andrew J Viterbi. Error bounds for convolutional codes and an asymptotically optimum decoding algorithm. Information Theory, IEEE Transactions on, 13(2):260-269, 1967.

[78] Texas Instruments. Viterbi Decoding Techniques in the TMS320C54x Family, aug. 2001.

[79] Shai Shpigelblat. Implementing the viterbi algorithm in today's digital communications systems. jul. 2009.

[80] Freescale. GSM Soft-Decision Viterbi Decoder, oct. 2002.

[81] Timo Vogt and Norbert Wehn. A reconfigurable application specific instruction set processor for convolutional and turbo decoding in a sdr environment. In Proceedings of the conference on Design, automation and test in Europe, DATE '08, pages 38-43, New York, NY, USA, 2008. ACM.

[82] M. Woh, Yuan Lin, and etc. From soda to scotch: The evolution of a wireless baseband processor. In Microarchitecture, 2008. MICRO41. 2008 41st IEEE/ACM International Symposium on, pages 152 -163, nov. 2008.

[83] Matlab 2012b LTE PHY Downlink with Spatial Multiplexing. Url http://www.mathworks.se/help/comm/examples/ltephy-downlink-with-spatial-multiplexing.html. Last access: 22nd October, 2013.

[84] Matlab LTE Downlink Physical Channel processing. Url http:/ / www.mathworks.com/matlabcentral/fileexchange/34279. Last access: 22nd October, 2013.

[85] Ingemar Ragnemalm and Andréas Karlsson. Computing The Euclidean Distance Transform on the ePUMA Parallel Hardware. In Computer Graphics, Visualization, Computer Vision and Image Processing 2011, pages 228-232, 2011. 
[86] Ingemar Ragnemalm and Dake Liu. Adapting the ePUMA Architecture for Hand-held Video Games. International Journal of Computer Information Systems and Industrial Management Applications, 4:153160, 2012.

[87] Jens Ogniewski, Andréas Karlsson, and Ingemar Ragnemalm. Texture compression in memory and performance-constrained embedded systems. In Computer Graphics, Visualization, Computer Vision and Image Processing 2011, pages 19-26, 2011.

[88] Wenbiao Zhou, Zhaoyun Cai, Ruiqiang Ding, Chen Gong, and Dake Liu. Efficient sorting design on a novel embedded parallel computing architecture with unique memory access. Computers \& Electrical Engineering, 39(7):2100-2111, 2013.

[89] Zhenyu Liu, Hongkai Wang, Qunfang Xie, Yanjun Zhang, Wenbiao Zhou, and Dake Liu. Implementation of prime-point dfts with programable processors. In Systems and Informatics (ICSAI), 2012 International Conference on, pages 1693-1697, 2012.

[90] OpenCores. Wishbone b4: Wishbone system-on-chip (soc) interconnection architecturefor portable ip cores. 2010.

[91] Brucek Khailany, William J Dally, Andrew Chang, Ujval J Kapasi, Jinyung Namkoong, and Brian Towles. Vlsi design and verification of the imagine processor. In Computer Design: VLSI in Computers and Processors, 2002. Proceedings. 2002 IEEE International Conference on, pages 289-294. IEEE, 2002.

[92] Arteris. Network on chip (noc) interconnect technology for socs. URL http://www.arteris.com/technology, Last access: 22nd October 2013. 
Part IV

Appendix 



\section{Appendix A \\ Master C Library}

This part describes the basic library functions for using the master core to control the data communication, synchronization and computing on SIMD coprocessors. 
Kernel_Load Load kernel to a SIMD coprocessor's scalar program memory

C function void Kernel_Load(int id, int scalar_base, int scalar_bytes);

Inputs id: the id number of the target SIMD processor.

scalar_base: pointer to the scalar program data.

scalar_bytes: the size of scalar program to be loaded

to SIMD coprocessor's scalar program memory, the size should be in number of bytes.

Description This function will load scalar program from the main memory to SIMD coprocessor's scalar program memory. It performs the following steps of operations:

1. Synchronize with DMA controller of the STAR network.

2. Synchronize with the target SIMD coprocessor (only IDLE mode allows kernel load)

3. Configure task 0 of DMA task memory for kernel loading.

4. Start the DMA controller from task 0. 
Kernel_Load2 Load kernel to a SIMD coprocessor's scalar program memory and VPE program memory

C function void Kernel_Load2(int id, int scalar_base, int scalar_bytes, int vpe_base, int vpe_bytes);

Inputs id: the id number of the target SIMD. scalar_base: pointer to the scalar program data. scalar_bytes: the size of scalar program to be loaded to SIMD scalar program memory, the size should be in number of bytes.

vpe_base: pointer to the vpe program data. vpe_bytes: the size of vpe program to be loaded to SIMD VPE program memory, the size should be in number of bytes.

Description This function will load scalar program and VPE program from the main memory to a SIMD coprocessor's scalar program memory and VPE program memory. It performs the following steps of operations:

1. Synchronize with DMA controller of the STAR network.

2. Synchronize with the target SIMD (only IDLE mode allows kernel load)

3. Configure task 0 of DMA task memory for kernel loading. Configure DMA task 1 for VPE program loading. Link DMA task 0 and task 1 to be completed by one DMA transaction.

4. Start the DMA controller from task 0. 
Kernel_Load_VPE Load VPE program from main memory to SIMD VPE program memory

\begin{tabular}{ll}
\hline C function & void Kernel_Load_VPE(int id, int vpe_base, int \\
& vpe_bytes, int vpe_base, int vpe_bytes ); \\
Inputs & id: the id number of the target SIMD. \\
& vpe_base: pointer to the vpe program data. \\
& vpe_bytes: the size of vpe program to be loaded \\
& to SIMD VPE program memory, the size should \\
& be in number of bytes. \\
& This funtion will load VPE program from the \\
& main memory to a SIMD coprocessor's VPE pro- \\
gram memory. It performs the following steps & of operations:
\end{tabular}

1. Synchronize with DMA controller of the STAR network.

2. Synchronize with the target SIMD (only IDLE mode allows kernel load)

3. 3. Configure task 0 of DMA task memory for VPE program.

4. Start the DMA controller from task 0. 
Kernel_BroadcastLoad Broadcast the same scalar program to multiple SIMD scalar program memory

C function void Kernel_BroadcastLoad(int mask, int scalar_base, int scalar_bytes);

Inputs mask: mask the target SIMD coprocessors to load the program, for example, mask = 0x0F selets SIMD 0,1,2,3, mask $=0 \times 81$ selects SIMD 0 and7.

scalar_base: pointer to the scalar program data.

scalar_bytes: the size of scalar program to be loaded to SIMD scalar program memory, the size is in number of bytes.

Description This function will load scalar program from the main memory to multiple SIMD coprocessor's scalar program memories by one DMA transaction. It performs the following steps of operations:

1. Synchronize with DMA controller of the STAR network.

2. Synchronize with the target SIMD (only IDLE mode allows kernel load)

3. 3. Configure task 0 of DMA task memory for VPE program.

4. Start the DMA controller from task 0. 
Kernel_BroadcastLoad2 Broadcast the same scalar program and VPE program to multiple SIMD scalar program memory and VPE program memory

C function void Kernel_BroadcastLoad(int id, int s-
calar_base, int scalar_bytes, int vpe_base, int ope_bytes);

Inputs

id: the id number of the target SIMD.

scalar_base: pointer to the scalar program data.

scalar_bytes: the size of scalar program to be loaded to SIMD scalar program memory, the size should be in number of bytes. vpe_base: pointer to the vpe program data.

vpe_bytes: the size of vpe program to be loaded to SIMD VPE program memory, the size should be in number of bytes.

Description This function will load scalar program and VPE program from the main memory to multiple SIMD scalar program memory by one DMA transaction. It performs the following steps of operations:

1. Synchronize with DMA controller of the STAR network.

2. Synchronize with the target SIMD (only IDLE mode allows kernel load)

3. 3. Configure task 0 of DMA task memory for VPE program.

4. Start the DMA controller from task 0. 
Wait_Star Synchronize with the central DMA controller of the Star network.

C function void Wait_Star(void);

Description This function synchronizes with the DMA controller of the Star network. It only returns when the DMA is in IDLE state.

Kernel_Start Start a SIMD coprocessor.

C function void Kernel_Start(int id);

Description This function starts a SIMD processor. The scalar core of the SIMD starts from SPM address 0 . The execution of VPE core is controlled by the scalar core. The scalar program memory should be initialized with correct program data before calling the start function.

Kernel_Wait Synchronize with a SIMD coprocessor.

C function void Kernel_Wait(int id);

Input id: the target SIMD ID.

Description This function synchronize with SIMD execution. It only returns when the SIMD is in IDLE state. A SIMD can go to IDLE state by writing to the STOP register: $\mathrm{STOP}=1$; 


\section{Appendix B \\ SIMD Scalar Controller C \\ Library}

This part describes the basic library functions for using SIMD scalar controller to control the SIMD local memory system to perform memory switch, PPU communication, and synchronization. 
Switch_LVM Switch the LVM memory connection.

C function void Switch_LVM(int scalar, int m0, int m1, int ppu);

Inputs scalar: select the LVM to connect to the scalar core.

$\mathrm{m} 0$ : select the LVM to connect to port 0 of VPE

$\mathrm{m} 1$ : select the LVM to connect to port 1 of VPE

ppu: select the LVM to connect to the PPU

Description This function switch the LVM connection to the memory ports of scalar core, VPE core, and PPU.

The value should be one of $0,1,2,0 x F, 0 x F$ means no connection.

Star_Send_1D Send 1D sequential data from LVM to main memory through the STAR network.

C function void Star_Send_1D(int base, int bytes);

Inputs base: the base addres of the sequential data in LVM. bytes: the size of the data block to be transferred.

Description This function send a continuous data block in the local LVM to the Star network. The data will be collected by the master core by issuing a DMA transfer to move the data to main memory.

Star_Receive_1D Receive 1D sequential data from the Star network and store the data into local LVM.

C function void Star_Receive_1D(int base);

Input base: the base address in the local LVM to store the data block.

Description This function receives a continuous data block from the STAR network and store it in the local LVM from the base address. The size of the data stream is not required since it is determined by the Star data packet. 
Ring_Send_1D Send 1D sequential data from LVM to another SIMD through the RING network.

C function void Ring_Send_1D(int dest, int base, int bytes);

Inputs dest: specify the destination SIMD.

base: the base address of the data block in the local LVM.

bytes: the size of the data block to be transferred.

Description This function sends a continuous data block in the local LVM to the target SIMD coprocessor through the RING network.

Ring_Broadcast_1D Send 1D sequential data from LVM to multiple SIMD coprocessors through the RING network.

\begin{tabular}{ll}
\hline C function & void Ring_Send_1D(int mask, int base, int bytes); \\
Inputs & mask: specify the destination SIMD coproces- \\
sors. \\
base: the base address of the data block in the \\
local LVM. \\
bytes: the size of the data block to be trans- \\
ferred. \\
This function sends a continuous data block in \\
the local LVM to the masked SIMD coproces- \\
sors through the RING network.
\end{tabular}


Ring_Receive_1D Receive 1D sequential data from the source SIMD coprocessor and store the data into local LVM.

C function Inputs

Description void Ring_Receive_1D(int source, int base); source: specify the source SIMD coprocessor. base: the base address in the local LVM to store the data block.

This function receives a continuous data block from the source SIMD coprocessor through the RING network and store it in the local LVM from the base address. The size of the data stream is not required since it is determined by the source.

Wait_PPU Synchronize with PPU communication.

C function void Wait_PPU(void);

Description This function synchronizes with the PPU communication. It only returns when the PPU finishes all the data transfer tasks. Should not switch LVM when PPU has ongoing task. 